\title{
Ranking Sustainable Projects through an Innovative Hybrid DEMATEL-VIKOR Decision-Making Approach Using Z-Number
}

\author{
Armin Akhavein (D), Ali RezaHoseini (D), AmirMohammad Ramezani (D), \\ and Morteza BagherPour
}

School of Industrial Engineering, Iran University of Science \& Technology, Tehran, Iran

Correspondence should be addressed to Ali RezaHoseini; ali_rezahoseini@ind.iust.ac.ir

Received 29 December 2020; Revised 18 February 2021; Accepted 20 March 2021; Published 12 April 2021

Academic Editor: Valeria Vignali

Copyright (C) 2021 Armin Akhavein et al. This is an open access article distributed under the Creative Commons Attribution License, which permits unrestricted use, distribution, and reproduction in any medium, provided the original work is properly cited.

\begin{abstract}
According to the heavy reliance of economic growth on environmental and social matters, sustainable development has turned into one of the main strategies of the organizations associated with selecting the project basket. Moreover, market conditions, rapid global changes in several aspects, uncertainty in intellectual judgments of the experts, and many other factors have increased uncertainty in problems of this kind. Accordingly, the main objective of this paper is to develop a mixed decision-making approach (DEMATEL-VIKOR) with the aim of ranking and evaluating suggested projects considering sustainability indices in a "mass production and infrastructural" company under uncertainty, while also taking reliability (Z-number information) into account. Given the existing uncertainty in the opinions of experts and previous data, fuzzy approach and Z-number information have been employed for weighting the filtered sustainability indices that are aligned with the objectives of the considered company (Z-DEMATEL), as well as ranking candidate projects (Z-VIKOR), which contribute to the main innovation of the present research. In addition, for scoring in the Z-number state, a novel definition of linguistic variable (fuzzy linguistic variable considering probabilities) has been put forward. The suggested approach was solved by an expert in two numerical examples in three certain, fuzzy, and Z-number scenarios, the results of which show that different results are obtained for different scenarios. Apart from that, given that Z-number takes the probability of reliance on the opinions of experts into consideration, the more the approach moves from being certain towards the fuzzy state and the Z-number state, the more the results approach the real world, hence yielding more reliable results. Therefore, results obtained from the proposed Z-DEMATEL-VIKOR approach are far more reliable. Moreover, significance weights in the Z-number scenario are 39\%, 26\%, and 36\% in the economic, social, and environmental dimensions, respectively, which is indicative of the importance of social and environmental aspects apart from the economic dimension.
\end{abstract}

\section{Introduction}

The proper project selection is an important task in many organizations, which demonstrates the importance of a systematic and comprehensive approach for selecting a project portfolio. However, project portfolio selection issues are inherently complex issues with multiple quantitative and qualitative criteria and often conflicting, such as business objectives, benefits and costs, and limited resources [1]. The existence of different and influential criteria and the multiplicity of options have led to a more systematic and regular project selection process in recent years. Most methods and techniques for the selection of project portfolios focus on financial criteria [2]. Economic analysis is the most common criterion used in the project selection process [1]. The results of a project have shortterm and long-term effects on social, economic, and environmental aspects; therefore, the third generation of new criteria has been evolved as sustainable development. Paying attention to the principles of sustainable development and considering social and environmental criteria along with economic criteria will lead to the selection of a sustainable and balanced portfolio [3]. Each organization seeks appropriate short-term and long-term plans to 
achieve the most optimal and profitable project from the available projects. Valuable resources and funds of any organization are not sufficient for trial and error in implementing all proposed projects. Hence, scientific, controllable, and management procedures and mechanisms regarding the selection, prioritization, and classification of projects have to be used. As a result, the direction of past and present works and activities is simpler to evaluate. Given the criteria for evaluating project selection are largely ambiguous and qualitative, therefore multidisciplinary decision-making approaches can be used [4]. Deciding on technical issues has a variety of complexities and dimensions. This complexity includes quantitative and qualitative parameters and criteria, and in mental and nondiscrete cases, achieving definite judgments is difficult. Therefore, decisions are always attached with uncertainty [5]. Hence, to get closer to the real world, fuzzy logic is used in these issues to reflect the views of technical experts as much as possible and to a large extent remove the limitations in their views on such issues [6]. Then again, as moving toward reality, probability and uncertainty in fuzzy numbers need to be considered [7]. Z-number is a subset of fuzzy sets, which involves the probability level in fuzzy numbers because the judgments of experts and the linguistic scoring variable of experts always include uncertainties [8]. The use of multicriteria decision-making methods for optimum project selection and ranking projects according to the criteria is very important. Many multiobjective decision-making methods aim for calculating the weight of the criteria, and others aim at ranking projects. DEMATEL is a well-known and comprehensive method for obtaining a structural model that provides the interrelationships between real-world complex criteria. The DEMATEL method is more advantageous than other techniques, such as the analytical hierarchy process (AHP) because the interdependence between the criteria of a system through the causal diagram in traditional techniques has been overlooked. The fuzzy DEMATEL method examines the interrelationships between criteria and determines the effectiveness or influence of the criteria (in other words, cause and effect criteria) and the importance weight of criteria by the total communication matrix. Therefore, in this study, in addition to fuzzy numbers, the reliability of these numbers has also been considered and the Z-DEMATEL approach has been used to weigh the sustainability criteria to rank the projects in order to reach more reliable answers [9]. Also, fuzzy VIKOR is one of the multicriteria decision-making methods that aim to select and rank the best projects. This method is similar to the VIKOR method but is related to fuzzy numbers and fuzzy information. The use of fuzzy information makes the results more accurate due to the ambiguity and uncertainty of the problem. In this study, the goal is to rank projects based on sustainability criteria [10]. Therefore, in addition to theoretical views of fuzzy numbers for ranking projects, using the Z-VIKOR approach, the level of these numbers' reliability is considered to approach the real world.
Given the significance of the mentioned issue: Is sustainable development a basis for defining and carrying out projects? Are sustainable subcriteria adequately selected for the selection of appropriate projects? Is the weighting of the subcriteria being properly done? Do the selected projects meet the requirements of the objectives of sustainable development? How should available projects be selected so that the objectives of sustainable development can be met? How properly are projects ranked using the existing ranking methods? Are uncertainties in judgments of the experts affecting the ranking of projects? Is there an approach that can bring uncertainties in scoring the questionnaires closer to reality?

In this paper, Section 2 shares out theoretical principles (definitions and terms) of fuzzy uncertainty and Z-number approach and a review of the previous literature in selecting and ranking the project portfolio. Finally, the articles are summarized in a table, and based on that, the research gap and the desired innovations for the research are expressed. Section 3 first identifies and filters sustainability criteria, and then, given the probabilistic linguistic variable associated with the Z-number approach, two new linguistic variables are defined for use in pairwise comparison matrices and scoring decision matrices. Based on the defined variable, the Z-DEMATEL approach for weighting sustainability dimensions and criteria and the Z-VIKOR approach for project ranking are introduced. In Section 4, first, the data related to pairwise comparisons of sustainability dimensions and pairwise comparisons of sustainability criteria for their weighting according to the defined linguistic variable of Znumber and data related to project scoring based on sustainability criteria are obtained. Then, the solution of the ZDEMATEL and Z-VIKOR integrated approach is demonstrated. In Section 5, based on the results obtained in Section 4 , analyses related to the values of weight values, criteria of sustainability, and project ranking in Z-number conditions and its comparison with deterministic and fuzzy conditions are discussed. In Section 6, the findings are summarized, and the managerial perspective of this research, the innovations in mind, and future suggestions are presented.

Therefore, considering the importance of the mentioned topics, the innovations of this study can be mentioned as follows:

(1) Identify and classify the most important sustainability criteria in order to select and rank sustainable projects through the study of books and articles, as well as filter them for review in this study.

(2) Define two new linguistic variables for Z-number which use in pairwise comparison matrices and scoring decision matrices.

(3) Given uncertainties in intellectual judgments of experts, Z-number information is used in the DEMATEL approach for weighting of sustainable criteria and their associated influence and be-influence. Apart from that, Z-number information is also used to rank and select sustainability projects, followed by being 
compared with results obtained from different scenarios (certain, fuzzy, and Z-number)

\section{Literature Review}

2.1. Ranking and Selecting Project Portfolio Importance. A portfolio is a collection of projects, programs, or other works that have been put together to facilitate the effective management and the estimation of strategic business objectives. Portfolio is within the organization and includes a set of current components, schedules, and initiatives for the future [11]. RezaHoseini et al. presented a linear multiobjective model, which is capable of dealing with a sustainable project portfolio selection problem and allows for the split of projects so that a part of it can be postponed to another time. Their model is aimed at selecting and scheduling projects based on time-dependent criteria as well as budget and resource constraints, solved via a hybrid multicriteria decision-making approach. All results they have obtained prove the effectiveness of their proposed strategy [12]. Outlining the importance of a proper contract in project management, Mahmoudi et al. have conducted a research work, which suggests a comprehensive model for risk management in outsourced construction project contracts. In this respect, the authors have first identified different types of contracts and the involved risks have been introduced as the criteria. The contracts have then been prioritized using the best-worst method (BWM) along with grey relation analysis (GRA) to prioritize the alternatives. Their results have shown that their strategy can be successfully applied to a diverse range of projects [13]. All portfolio components have certain common characteristics: (1) they represent the investment or program chosen by the organization; (2) they are aligned with the strategic goals of the organization; (3) they usually have distinctive features that allow the organization to categorize them for effective management; and (4) they are quantitative so they can be measured, ranked, and prioritized. In addition, the project portfolio selection is a complex decision problem which is composed of tangible and intangible criteria. Among the criteria that have been taken into consideration nowadays, sustainability and strategic criteria account for the main criteria in this field nowadays. Strategic objectives and changing them have an impact on the view of the managers on the project [14]. They have prioritized the proposed projects according to the strategic objectives of the companies, and if these strategic goals are changed, the priority of projects for selecting and performing will be altered. Therefore, the organization should determine a conceptual framework consisting of critical metrics that align with the organization's strategic goals since these criteria have a direct impact on the efficiency of decision-making of project selection [15]. Wu et al. have established a fuzzy multicriteria decision-making model for the optimization of renewable energy project portfolios. They have identified sustainable criteria and the associated subcriteria first, and the fuzzy weights of which have been then obtained using the IT2FAHP method. The Pareto-optimal set of the evaluated objectives has been finally obtained via a nondominated sorting genetic algorithm-II (NSGA-II). Their suggested approach has been validated by a case study in Southeast China [16]. In another research work by Wu et al., a multicriteria decisionmaking approach has been developed to select the optimal distributed energy generation project portfolio, taking uncertainty and project interaction into consideration. Firstly, the IT2F-AHP method has been used to determine the weights of the criteria, after which the optimal portfolio of the mentioned projects has been obtained via an NSGA-II. The method has been also tested on a case study to further illustrate its effectiveness [16]. In 2018, a study was carried out by $\mathrm{Wu}$ et al. where an integrated framework was proposed via a combination of fuzzy multiattribute decisionmaking and fuzzy multiobjective programming to address problems associated with large-scale rooftop photovoltaic project portfolio selection including uncertainties in decision-making and interactions between projects. Firstly, the values of the attributes, objective functions, and constraints have been represented as triangular intuitionistic fuzzy numbers, describing inherent uncertainties. Afterwards, the weights of the attributes are determined by AHP, followed by obtaining the Pareto-optimal set of objectives via the NSGA-II. Their proposed strategy has been tested on a case study in Zhejiang for further validation [17].

2.2. Sustainable Importance and Its Application. Various definitions of sustainable and different sustainable criteria have been presented in which sustainability perspective has consisted of economic value, environmental issues, and social responsibility and finding a balance between them $[18,19]$. Given that these three pillars of sustainability affect the selection and implementation of projects, many studies have adopted them in project selection and scheduling [20-22]. Due to the importance of environmental protection, companies and organizations have been compelled to select and employ projects that have less negative effects on the environment by using sustainability issues, and since project investment also can lead to boosting the economy, the economic issue takes a key role in project selection problems. In addition, social aspects are another sector of the sustainable issue that affects projects performance. Traditional project selection has paid attention to just financial criteria and profitability without considering sustainability while, these days, many researchers have also considered sustainability in their studies since a combination of financial and sustainability criteria can trigger to enhance organizations' productivity and competitiveness and can affect project success and project management $[12,19,22]$. Duan et al. have developed an integrated green supplier selection and order allocation (BSSOA) model to select appropriate suppliers along with the reasonable allocation of order quantities via a combination of linguistic Znumbers and the alternative queuing method (AQM) together with the multiobjective line programming (MOLP) model. The performances of suppliers have been first evaluated by linguistic Z-numbers expressed by decisionmakers, followed by the calculation of weights of the criteria. The MOLP model is then used to represent the optimal order 
quantity for the green suppliers based on the priority values specified in the previous stages [23]. Chang and Cheng have conducted a study to develop a multiattribute decisionmaking model which is capable of evaluating the sustainable development of manufacturing SMEs in Taiwan. For the selection of sustainability indicators, the fuzzy Delphi method (FDM) has been used, the performance evaluation of which has been developed by a GRA approach. Ultimately, critical factors that influence performance have been identified via a sensitivity analysis [24]. In another work by Ikram et al., the effectiveness of taking environmental management systems (EMS) on the sustainability of IMSs has been investigated. In their study, 211 manufacturing corporations have been selected in Pakistan, where a stakeholder-weighted CSR index together with an equalweighted CRS index has been employed so that the performance of the mentioned companies would be measured. The results have shown that EMS adopters have shown a superior performance compared to non-EMS adopters in terms of mean CFR performance, suggesting that EMS adoption can effectively address several issues faced by the corporations [25]. In a research conducted in 2020 by Ikram et al., a systematic framework has been developed that prioritizes barriers to implementing integrated management systems (IMSs). The barriers have been identified using the literature, the weights of which have been calculated by AHP, followed by applying a grey preference by similarity to ideal solution (GTOPSIS) to rank the alternatives. Finally, the robustness of their strategy has been evaluated by a sensitivity analysis [26]. In a study conducted by Ikram et al. in 2020, a number of challenges that could potentially affect corporate sustainability have been highlighted so as to prioritize significant indicators of sustainability in the literature, after which the fuzzy analytical hierarchy process (FAHP) has been employed to help determine the weights of the associated criteria. The COVID-19 pandemic has been taken into consideration as a sustainability attributor in their research [27]. Due to the importance of sustainable strategic criteria, project evaluation, and ranking of projects in portfolio project selection, in the following, studies conducted in this field are reviewed.

\subsection{Ranking and Selecting Project Portfolio Using Multicriteria} Decision-Making Approaches. Various methods have been proposed for selecting a project portfolio. Based on a study [28] that has been done on different types of project selection methods, more than one hundred different methods for evaluating and selecting projects have been discussed [29]. In 2000, a study proposed a way to select information system projects that reflect the interdependence between evaluation criteria and candidate projects using the analytical network process (ANP), in order to gather group opinions on an issue [30]. In a 2002 paper, the analytical network process (ANP) was used to make decisions about $R \& D$ projects. In this study, the decision-maker tries to choose one of the two options of the new system or speed up the printing of the existing system [31]. In an article in 2004, the use of fuzzy calculations for the decision-making process is presented. In the project selection process, decision-makers must make their own decisions based on different criteria and different expert judgments. The problem is solved using a limited fuzzy AHP model, which is a special case of fuzzy AHP proposed by other researchers such as [32-34]. In 2005, an article was published on the selection of the R\&D project, which searched for opportunities and evaluated various projects qualitatively and quantitatively. Given the risk and uncertainty of R\&D projects, this paper also demonstrates the application of the ANP network method to fuzzy cost analysis in R\&D project selection issues [35]. Another article in 2005 provides an example of how experiments can be prioritized using a five-step project selection model. Questionnaires are filled in by a group of construction specialists [36]. An article in 2008 presents a fuzzy analytical hierarchy process (FAHP) method and uses deterministic judgment. It is shown how evaluation criteria change under different risk conditions [37]. In a 2010 paper, the analytical network process (ANP) is applied to select photovoltaic (PV) solar cell power projects. In this article, the CEO of an important Spanish company operating in the energy market intends to decide on the best PV project out of four candidate projects [38]. An article in 2010 provided a simple approach for evaluating options for the National Iranian Oil Company using six criteria comparing investment options as a criterion in AHP and fuzzy TOPSIS techniques. AHP is used to analyze the structure of the project selection problem and to determine the weight of the criteria and the fuzzy TOPSIS method to obtain the final ranking [39]. In a 2011 Spanish article, the author sought to assess the compliance of the selection criteria for renewable energy projects with government policies. The VIKOR method introduces the ranking index based on a specific "proximity" criterion to the "ideal" solution. This method is combined with hierarchical analysis (AHP) to measure the importance of different criteria [40]. In 2011, the authors of the Iranian paper defined six criteria for selecting the optimal project as cost criteria (risk and repayment period) and profit criteria (profitability, compatibility with company goals, flexibility, and sustainability). In this paper, the implementation of an organized framework for project portfolio selection through the VIKOR technique using linguistic (fuzzy) terms as well as weight calculation of the criteria importance and ranking the projects is presented in descending order [41]. An article was published in 2012 providing a fuzzy ELECTRE approach to prioritize the most effective projects, improving decisionmaking [42]. Another paper in 2013 presented a new group decision-making approach with new fuzzy criteria, FMCGDM (fuzzy multiple criteria group decision-making), for selecting a sustainable project. First, the framework is proposed, including the economic, social, and environmental impacts of investment, strategic correlation, organizational readiness, and investment risk to select a sustainable project. Since the importance of the proposed framework criteria can hardly be found through several groups with different views and preferences, a goal programming (GP) is presented with multiplicity and fuzzy states [2]. In another 2014 article, AHP (analytical hierarchy process) and ANP (analytical network process) assist the 
TABLE 1: Summary of previous articles.

\begin{tabular}{|c|c|c|c|c|c|c|c|c|}
\hline \multirow{2}{*}{ No. } & \multirow{2}{*}{ Reference } & \multirow{2}{*}{ Year } & \multirow{2}{*}{ Portfolio } & \multicolumn{3}{|c|}{ Sustainability dimensions } & \multirow{2}{*}{ Uncertainty } & \multirow{2}{*}{ Method } \\
\hline & & & & Economic & Social & Environment & & \\
\hline 1 & {$[30]$} & 2000 & Information system & $\checkmark$ & & & & ANP \\
\hline 2 & {$[31]$} & 2002 & $\mathrm{R} \& \mathrm{D}$ & $\checkmark$ & & & & ANP \\
\hline 3 & {$[32]$} & 2004 & General & $\checkmark$ & & & Fuzzy & AHP \\
\hline 4 & {$[35]$} & 2005 & $\mathrm{R} \& \mathrm{D}$ & $\checkmark$ & & & Fuzzy & ANP \\
\hline 6 & {$[36]$} & 2005 & Construction & $\checkmark$ & & & & ANP \\
\hline 7 & {$[37]$} & 2008 & Technology development & $\checkmark$ & $\checkmark$ & & Fuzzy & F-AHP \\
\hline 8 & {$[38]$} & 2010 & Solar energy & $\checkmark$ & $\checkmark$ & $\checkmark$ & & ANP \\
\hline 9 & [39] & 2010 & Oil & $\checkmark$ & & & Fuzzy & F-AHP + F-TOPSIS \\
\hline 10 & {$[40]$} & 2011 & Energy & $\checkmark$ & $\checkmark$ & $\checkmark$ & & VIKOR + AHP \\
\hline 11 & [41] & 2011 & General & $\checkmark$ & $\checkmark$ & $\checkmark$ & Fuzzy & F-VIKOR \\
\hline 12 & {$[42]$} & 2012 & General & $\checkmark$ & & & Fuzzy & F-ELECTRE \\
\hline 13 & {$[2]$} & 2013 & General & $\checkmark$ & $\checkmark$ & $\checkmark$ & Fuzzy & F-TOPSIS \\
\hline 14 & [43] & 2014 & Thermal solar power plant & $\checkmark$ & & & & $\mathrm{AHP}+\mathrm{ANP}$ \\
\hline 15 & {$[44]$} & 2014 & Construction & $\checkmark$ & $\checkmark$ & $\checkmark$ & Fuzzy & F-AHP + F-TOPSIS \\
\hline 16 & {$[45]$} & 2015 & Six Sigma & & $\checkmark$ & & & ANP + DEMATEL \\
\hline 17 & {$[46]$} & 2016 & Urban Renovation & $\checkmark$ & $\checkmark$ & $\checkmark$ & Fuzzy & AHP + PROMETHEE \\
\hline 18 & {$[47]$} & 2018 & Railroad system & $\checkmark$ & $\checkmark$ & $\checkmark$ & & ANP \\
\hline 19 & [48] & 2018 & General & $\checkmark$ & & & Fuzzy & F-QFD + F-DEA \\
\hline 20 & [49] & 2019 & Urban Renovation & $\checkmark$ & $\checkmark$ & $\checkmark$ & & MADM + TOPSIS + AHP \\
\hline 21 & {$[50]$} & 2019 & General & $\checkmark$ & & & Fuzzy & F-AHP \\
\hline 22 & {$[51]$} & 2019 & Information technology & $\checkmark$ & $\checkmark$ & & Fuzzy & F-AHP \\
\hline 23 & {$[52]$} & 2019 & Flood control & $\checkmark$ & $\checkmark$ & $\checkmark$ & Fuzzy & F-AHP + F-VIKOR \\
\hline 24 & {$[53]$} & 2019 & General & $\checkmark$ & & & Fuzzy & F-TOPSIS + F-AHP \\
\hline 25 & {$[54]$} & 2019 & R\&D & $\checkmark$ & & & & MCDM \\
\hline 26 & {$[55]$} & 2019 & Scholarship & $\checkmark$ & $\checkmark$ & & & AHP + PROMETHEE II \\
\hline 27 & {$[56]$} & 2019 & $\mathrm{R} \& \mathrm{D}$ & $\checkmark$ & & & Intuitionistic fuzzy & IF-DEMATEL + IF-TOPSIS \\
\hline 28 & [20] & 2020 & Paper manufacturing & $\checkmark$ & $\checkmark$ & $\checkmark$ & & TOPSIS \\
\hline 29 & [57] & 2020 & Oil & $\checkmark$ & & & & MADM \\
\hline 30 & This Paper & 2020 & Construction & $\checkmark$ & $\checkmark$ & $\checkmark$ & Z-number & Z-DEMATEL + Z-VIKOR \\
\hline
\end{tabular}

board of a major Spanish solar energy investment company to decide whether to invest in specific solar thermal power plan, and help to determine the order of projects in the company's portfolio. The decision-making approach presented in this paper consists of three steps. In the first two steps, the CEO must decide whether to accept or reject a project according to a set of predetermined criteria by the technical team [43]. In a 2014 article, the authors sought new analytical tools for evaluating construction projects and their overall risks under incomplete and uncertain conditions (uncertainty). Their other goal is to categorize risks and predict their levels and then use them to advance strategies and deal with high-risk factors. The Relative Importance Index (RII) is used to prioritize project risks based on the data obtained. Then, construction projects are classified by fuzzy AHP method and fuzzy TOPSIS methods [44]. In a 2015 article, ANP (analytical network process) and DEMATEL-ANP were used in a public medical center to select the best Six Sigma project. ANP and DEMATEL-ANP methods are used to evaluate 6 Six Sigma projects by 3 strategies, 4 criteria, and 15 subcriteria [45]. A 2016 study presented an integrated approach to selecting urban renewal projects. The proposed method is a combination of the analytical hierarchy process (AHP) and PROMETHEE, to help construction companies choose the right project for urban renewal. AHP and PROMETHEE are used to find the weight of selection criteria and to rank alternative projects,

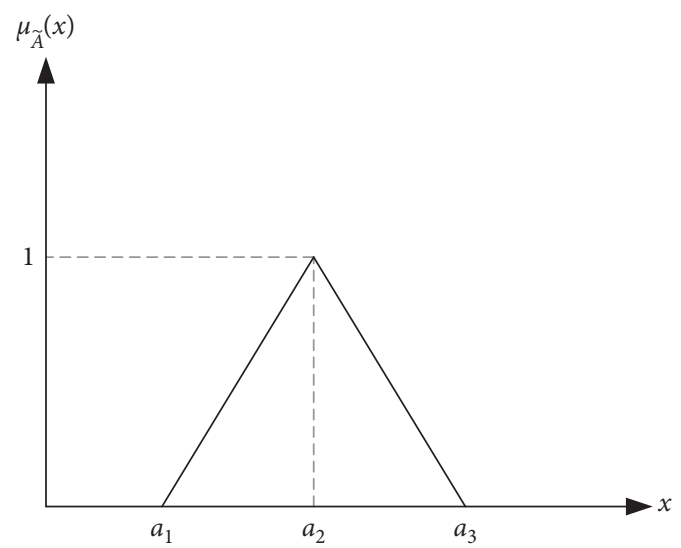

Figure 1: Triangular fuzzy number.

respectively [46]. In 2018, an article of Istanbul Metropolitan Municipality is tried to improve urban transportation by presenting the submitted projects of the Istanbul city transport system. The project evaluation criteria have been determined, and in this regard, rail system projects have been prioritized using fuzzy analytical networking process (ANP), which is one of the multicriteria decision-making methods [47]. In the 2018 paper, an approach is proposed that can simultaneously consider three important elements: (1) prioritization of selection criteria, (2) uncertainty in 


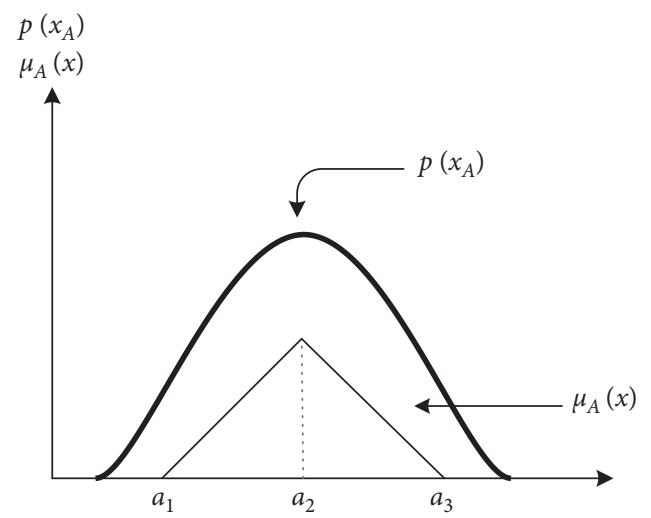

(a)

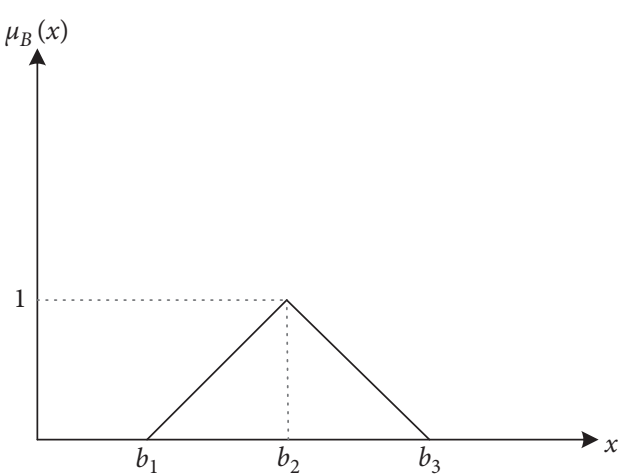

(b)

Figure 2: An example of Z-number.

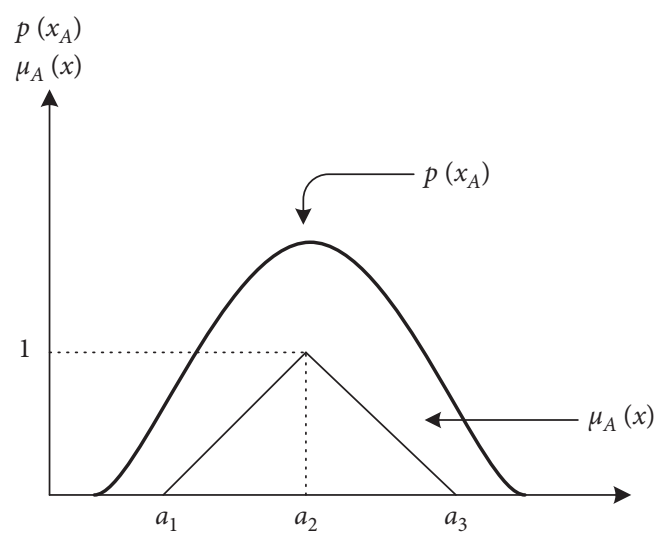

Figure 3: Distribution of $X$ probability and triangular membership function of event $A$.

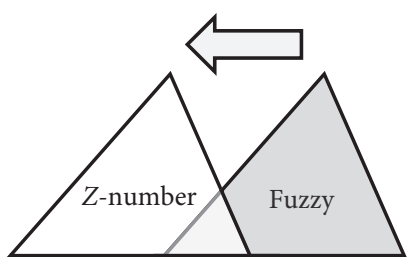

(a)

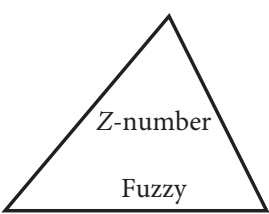

(b)

Figure 4: (a). Fuzzy number and its corresponding Z-number in the $\alpha<1$ condition. (b). Fuzzy number and its corresponding Z-number in the $\alpha=1$ condition.

TABLE 2: Linguistic variables used and their corresponding fuzzy numbers.

\begin{tabular}{lccc}
\hline Triangular fuzzy numbers & Deterministic equivalent & Notation & Linguistic variable \\
\hline$[0.00,0.00,0.25]$ & $\widetilde{1}$ & $($ VS) & Very small \\
{$[0.00,0.25,0.50]$} & $\widetilde{2}$ & $(\mathrm{~S})$ & Small \\
{$[0.25,0.50,0.75]$} & $\widetilde{3}$ & $(\mathrm{M})$ & Medium \\
{$[0.50,0.75,1.00]$} & $\widetilde{4}$ & $(\mathrm{~B})$ & Big \\
{$[0.75,1.00,1.00]$} & $\widetilde{5}$ & Very big \\
\hline
\end{tabular}

decision-making, and (3) interdependence of projects. The purpose of this paper is to present a report on an integrated approach that can address all three aspects simultaneously. The proposed method is a combination of quality performance development (QFD), fuzzy logic, and data envelopment analysis (DEA) and applied in a numerical example from the real world [48]. In an article in 2019, the main goal is to provide an integrated approach that uses 
TABLE 3: The linguistic variables used and their corresponding fuzzy numbers of Z-number in probabilistic conditions.

\begin{tabular}{|c|c|c|c|c|}
\hline Linguistic variable & Notation & Triangular fuzzy number & $\alpha$ & $\sqrt{\alpha}$ \\
\hline Unlikely & $(U)$ & {$[0.1,0.2,0.3]$} & 0.2 & 0.45 \\
\hline Fairly impossible & (FI) & {$[0.3,0.4,0.5]$} & 0.4 & 0.63 \\
\hline Weak & $(W)$ & {$[0.4,0.5,0.6]$} & 0.5 & 0.71 \\
\hline Maybe & $(M)$ & {$[0.5,0.6,0.7]$} & 0.6 & 0.77 \\
\hline Likely & $(L)$ & {$[0.7,0.8,0.9]$} & 0.8 & 0.89 \\
\hline Most likely & $(\mathrm{ML})$ & {$[0.8,0.9,1.0]$} & 0.9 & 0.95 \\
\hline Certainly & $(C)$ & {$[1.0,1.0,1.0]$} & 1 & 1 \\
\hline
\end{tabular}

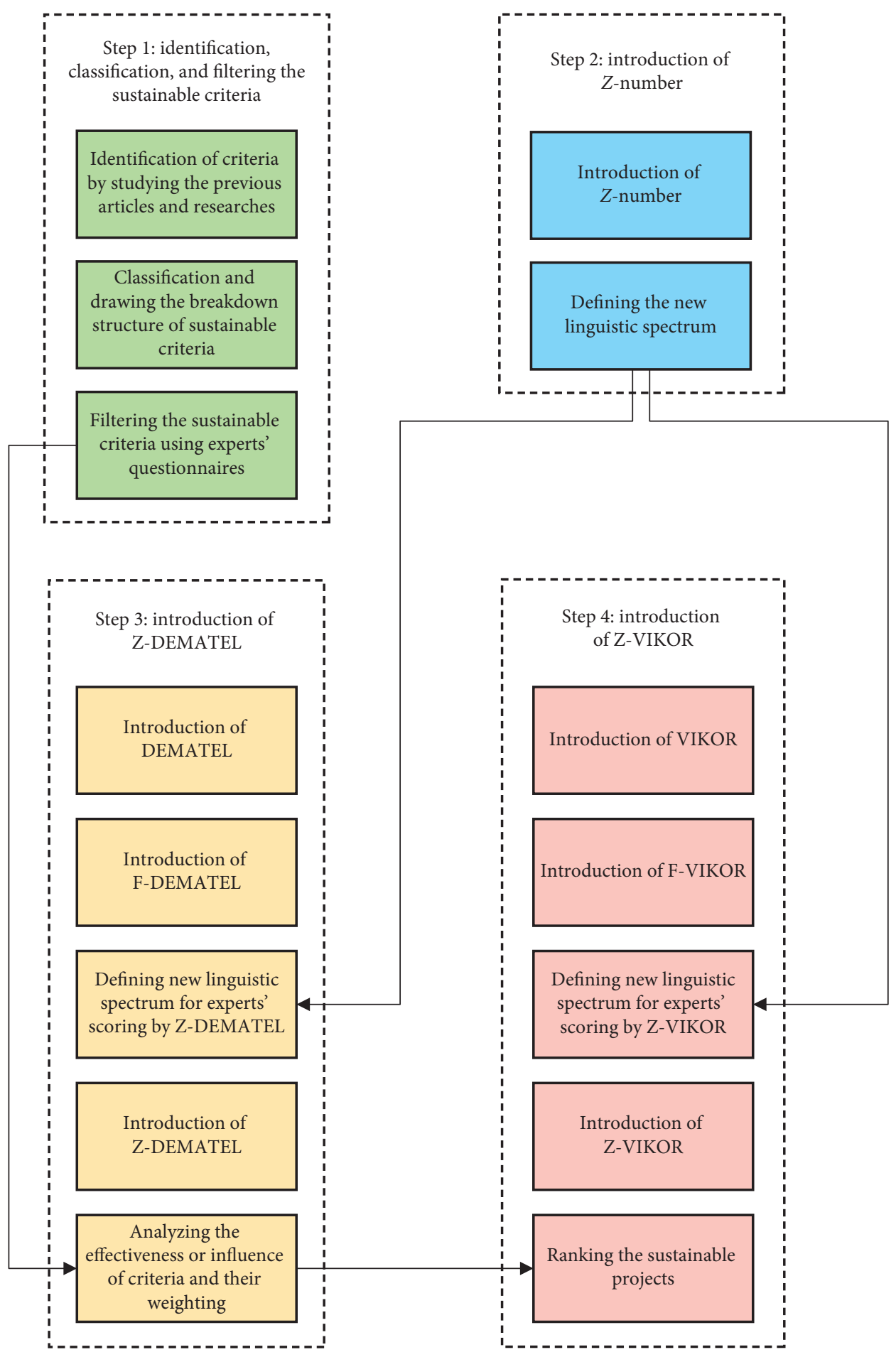

FIgURE 5: Research method process. 
TABLE 4: Initial list of extracted criteria.

\begin{tabular}{|c|c|}
\hline No. & Criteria \\
\hline 1 & Net worth of project implementation \\
\hline 2 & Basic infrastructures for project implementation \\
\hline 3 & Impact on the competitive market if the project is implemented \\
\hline 4 & Technology availability for project implementation \\
\hline 5 & Liquidity of company's investment to implement the project \\
\hline 6 & Employment rate in case of project implementation \\
\hline 7 & Project buildability \\
\hline 8 & Organizational readiness for project implementation \\
\hline 9 & Technology transfer and training rate in case of project implementation \\
\hline 10 & The level of necessity and urgency to implement the project \\
\hline 11 & Impact on working conditions in the community if the project is implemented \\
\hline 12 & The amount of durable material used to implement the project \\
\hline 13 & Probability of project success \\
\hline 14 & Knowledge of the company's project executive team \\
\hline 15 & The amount of synergy and homogeneity between the pillars of the project \\
\hline 16 & Satisfaction of employees and staff \\
\hline 17 & Improving the company's human resource capabilities from project implementation \\
\hline 18 & The amount of greenhouse gas production in the project \\
\hline 19 & The rate of return on investment from the project \\
\hline 20 & Amount of nonrenewable energy consumption in project \\
\hline 21 & Use of natural energy in the project \\
\hline 22 & Use of local workers in construction and operation of the project \\
\hline 23 & The impact on the global community and public opinion \\
\hline 24 & Investment return period from project implementation \\
\hline 25 & Expected revenue from project implementation \\
\hline 26 & The amount of water consumption in project \\
\hline 27 & The amount of water pollution resulting from the project \\
\hline 28 & The amount of soil pollution resulting from the project \\
\hline 29 & The amount of noise pollution resulting from the project \\
\hline 30 & Dissemination of particles and dust from the project \\
\hline 31 & The amount of unpleasant odors from the project \\
\hline 32 & The amount of recycling resulting from project implementation waste \\
\hline 33 & The amount of waste from project implementation \\
\hline 34 & The level of local suppliers and domestic production usage \\
\hline 35 & Commercial risk, technology risk, and project risk in case of project implementation \\
\hline 36 & The impact of sanctions on project implementation \\
\hline 37 & Alignment of project implementation with the prevailing environmental rules and regulations \\
\hline 38 & The advantage of project competitiveness \\
\hline 39 & Safety and health of employees and workers in case of project implementation \\
\hline 40 & Alignment of the project with the demands and needs of customers (employer) \\
\hline 41 & Customer satisfaction (employer) in case of project implementation \\
\hline 42 & Customer safety and health \\
\hline 43 & The level of community satisfaction with the implementation of the project \\
\hline 44 & Respect for local culture and distinctions if the project is implemented \\
\hline 45 & Acceptance of the community and its support for the implementation of the project \\
\hline 46 & Stakeholder participation in the project \\
\hline 47 & Reusability of material in case of end of project life \\
\hline 48 & Use of recyclable consumables in the project \\
\hline 49 & The willingness of unions, organizations, and the government to participate in the project implementation \\
\hline
\end{tabular}

TABLE 5: The linguistic variables used and their corresponding fuzzy numbers.

\begin{tabular}{lccc}
\hline Linguistic variable & Notation & Deterministic equivalent & Triangular fuzzy numbers \\
\hline No influence & $(\mathrm{N})$ & $\widetilde{0}$ & {$[0.00,0.00,0.25]$} \\
Very low influence & $(\mathrm{VL})$ & $\widetilde{1}$ & {$[0.00,0.25,0.50]$} \\
Low influence & $(\mathrm{L})$ & $\widetilde{3}$ & {$[0.25,0.50,0.75]$} \\
High influence & $(\mathrm{H})$ & $\widetilde{4}$ & {$[0.50,0.75,1.00]$} \\
Very high influence & $(\mathrm{VH})$ & {$[0.75,1.00,1.00]$} \\
\hline
\end{tabular}


TABLe 6: The linguistic variables used and their corresponding Z-numbers.

\begin{tabular}{|c|c|c|c|c|}
\hline linguistic variable & Notation & Deterministic equivalent & Fuzzy Number & Z-number \\
\hline No influence-unlikely & $(\mathrm{N}-\mathrm{U})$ & $\widetilde{0}$ & {$[0.00,0.00,0.25]$} & {$[0.00,0.00,0.11]$} \\
\hline Very low influence-unlikely & (VL-U) & $\tilde{1}$ & {$[0.00,0.25,0.50]$} & {$[0.00,0.11,0.22]$} \\
\hline Low influence-unlikely & $(\mathrm{L}-\mathrm{U})$ & $\tilde{2}$ & {$[0.25,0.50,0.75]$} & {$[0.11,0.22,0.34]$} \\
\hline High influence-unlikely & $(\mathrm{H}-\mathrm{U})$ & $\tilde{3}$ & {$[0.50,0.75,1.00]$} & {$[0.22,0.34,0.45]$} \\
\hline Very high influence-unlikely & $(\mathrm{VH}-\mathrm{U})$ & $\tilde{4}$ & {$[0.75,1.00,1.00]$} & {$[0.34,0.45,0.45]$} \\
\hline No influence-fairly impossible & $(\mathrm{N}-\mathrm{FI})$ & $\tilde{0}$ & {$[0.00,0.00,0.25]$} & {$[0.00,0.00,0.16]$} \\
\hline Very low influence-fairly impossible & (VL-FI) & $\tilde{1}$ & {$[0.00,0.25,0.50]$} & {$[0.00,0.16,32]$} \\
\hline Low influence-fairly impossible & $(\mathrm{L}-\mathrm{FI})$ & $\tilde{2}$ & {$[0.25,0.50,0.75]$} & {$[0.16,0.32,0.47]$} \\
\hline High influence-fairly impossible & $(\mathrm{H}-\mathrm{FI})$ & $\tilde{3}$ & {$[0.50,0.75,1.00]$} & {$[0.32,0.47,0.63]$} \\
\hline Very high influence-fairly impossible & (VH-FI) & $\tilde{4}$ & {$[0.75,1.00,1.00]$} & {$[0.47,0.63,0.63]$} \\
\hline No influence-weak & $(\mathrm{N}-\mathrm{W})$ & $\widetilde{0}$ & {$[0.00,0.00,0.25]$} & {$[0.00,0.00,0.18]$} \\
\hline Very low influence-weak & $(\mathrm{VL}-\mathrm{W})$ & $\tilde{1}$ & {$[0.00,0.25,0.50]$} & {$[0.00,0.18,0.35]$} \\
\hline Low influence-weak & $(\mathrm{L}-\mathrm{W})$ & $\tilde{2}$ & {$[0.25,0.50,0.75]$} & {$[0.18,0.35,0.53]$} \\
\hline High influence-weak & $(\mathrm{H}-\mathrm{W})$ & $\tilde{3}$ & {$[0.50,0.75,1.00]$} & {$[0.35,0.53,0.71]$} \\
\hline Very high influence-weak & $(\mathrm{VH}-\mathrm{W})$ & $\tilde{4}$ & {$[0.75,1.00,1.00]$} & {$[0.53,0.71,0.71]$} \\
\hline No influence-maybe & $(\mathrm{N}-\mathrm{M})$ & $\tilde{0}$ & {$[0.00,0.00,0.25]$} & {$[0.00,0.00,0.19]$} \\
\hline Very low influence-maybe & (VL-M) & $\tilde{1}$ & {$[0.00,0.25,0.50]$} & {$[0.00,0.19,0.39]$} \\
\hline Low influence-maybe & (L-M) & $\tilde{2}$ & {$[0.25,0.50,0.75]$} & {$[0.19,0.39,0.58]$} \\
\hline High influence-maybe & $(\mathrm{H}-\mathrm{M})$ & $\tilde{3}$ & {$[0.50,0.75,1.00]$} & {$[0.39,0.58,0.77]$} \\
\hline Very high influence-maybe & (VH-M) & $\tilde{4}$ & {$[0.75,1.00,1.00]$} & {$[0.58,0.77,0.77]$} \\
\hline No influence-likely & $(\mathrm{N}-\mathrm{L})$ & $\widetilde{0}$ & {$[0.00,0.00,0.25]$} & {$[0.00,0.00,0.22]$} \\
\hline Very low influence-likely & (VL-L) & $\tilde{1}$ & {$[0.00,0.25,0.50]$} & {$[0.00,0.22,0.45]$} \\
\hline Low influence-likely & $(\mathrm{L}-\mathrm{L})$ & $\tilde{2}$ & {$[0.25,0.50,0.75]$} & {$[0.22,0.45,0.67]$} \\
\hline High influence-likely & (H-L) & $\tilde{3}$ & {$[0.50,0.75,1.00]$} & {$[0.45,0.67,0.89]$} \\
\hline Very high influence-likely & (VH-L) & $\tilde{4}$ & {$[0.75,1.00,1.00]$} & {$[0.67,0.89,0.89]$} \\
\hline No influence-most likely & (N-ML) & $\tilde{0}$ & {$[0.00,0.00,0.25]$} & {$[0.00,0.00,0.24]$} \\
\hline Very low influence-most likely & (VL-ML) & $\tilde{1}$ & {$[0.00,0.25,0.50]$} & {$[0.00,0.24,0.47]$} \\
\hline Low influence-most likely & $(\mathrm{L}-\mathrm{ML})$ & $\tilde{2}$ & {$[0.25,0.50,0.75]$} & {$[0.24,0.47,0.71]$} \\
\hline High influence-most likely & (H-ML) & $\widetilde{3}$ & {$[0.50,0.75,1.00]$} & {$[0.47,0.71,0.95]$} \\
\hline Very high influence-most likely & (VH-ML) & $\tilde{4}$ & {$[0.75,1.00,1.00]$} & {$[0.71,0.95,0.95]$} \\
\hline No influence-certainly & $(\mathrm{N}-\mathrm{C})$ & $\tilde{0}$ & {$[0.00,0.00,0.25]$} & {$[0.00,0.00,0.25]$} \\
\hline Very low influence-certainly & (VL-C) & $\tilde{1}$ & {$[0.00,0.25,0.50]$} & {$[0.00,0.25,0.50]$} \\
\hline Low influence-certainly & $(\mathrm{L}-\mathrm{C})$ & $\tilde{2}$ & {$[0.25,0.50,0.75]$} & {$[0.25,0.50,0.75]$} \\
\hline High influence-certainly & $(\mathrm{H}-\mathrm{C})$ & $\tilde{3}$ & {$[0.50,0.75,1.00]$} & {$[0.50,0.75,1.00]$} \\
\hline Very high influence-certainly & (VH-C) & $\tilde{4}$ & {$[0.75,1.00,1.00]$} & {$[0.75,1.00,1.00]$} \\
\hline
\end{tabular}

TABLE 7: The linguistic variables used and their corresponding Z-numbers.

\begin{tabular}{|c|c|c|c|c|}
\hline Linguistic variable & Notation & Deterministic equivalent & Fuzzy number & Z-number \\
\hline Very small-unlikely & $(\mathrm{VS}-\mathrm{U})$ & $\widetilde{1}$ & {$[0.00,0.00,0.25]$} & {$[0.00,0.00,0.11]$} \\
\hline Small-unlikely & $(\mathrm{S}-\mathrm{U})$ & $\tilde{2}$ & {$[0.00,0.25,0.50]$} & {$[0.00,0.11,0.22]$} \\
\hline Medium-unlikely & $(\mathrm{M}-\mathrm{U})$ & $\tilde{3}$ & {$[0.25,0.50,0.75]$} & {$[0.11,0.22,0.34]$} \\
\hline Big-unlikely & $(\mathrm{B}-\mathrm{U})$ & $\tilde{4}$ & {$[0.50,0.75,1.00]$} & {$[0.22,0.34,0.45]$} \\
\hline Very big-unlikely & (VB-U) & $\widetilde{5}$ & {$[0.75,1.00,1.00]$} & {$[0.34,0.45,0.45]$} \\
\hline Very small-fairly impossible & (VS-FI) & $\tilde{1}$ & {$[0.00,0.00,0.25]$} & {$[0.00,0.00,0.16]$} \\
\hline Small-fairly impossible & $(\mathrm{S}-\mathrm{FI})$ & $\tilde{2}$ & {$[0.00,0.25,0.50]$} & {$[0.00,0.16,0.32]$} \\
\hline Medium-fairly impossible & (M-FI) & $\tilde{3}$ & {$[0.25,0.50,0.75]$} & {$[0.16,0.32,0.47]$} \\
\hline Big-fairly impossible & (B-FI) & $\tilde{4}$ & {$[0.50,0.75,1.00]$} & {$[0.32,0.47,0.63]$} \\
\hline Very big-fairly impossible & (VB-FI) & $\widetilde{5}$ & {$[0.75,1.00,1.00]$} & {$[0.47,0.63,0.63]$} \\
\hline Very small-weak & (VS-W) & $\tilde{1}$ & {$[0.00,0.00,0.25]$} & {$[0.00,0.00,0.18]$} \\
\hline Small-weak & $(\mathrm{S}-\mathrm{W})$ & $\tilde{2}$ & {$[0.00,0.25,0.50]$} & {$[0.00,0.18,0.35]$} \\
\hline Medium-weak & $(\mathrm{M}-\mathrm{W})$ & $\widetilde{3}$ & {$[0.25,0.50,0.75]$} & {$[0.18,0.35,0.53]$} \\
\hline Big-weak & $(\mathrm{B}-\mathrm{W})$ & $\tilde{4}$ & {$[0.50,0.75,1.00]$} & {$[0.35,0.53,0.71]$} \\
\hline Very big-weak & $(\mathrm{VB}-\mathrm{W})$ & $\widetilde{5}$ & {$[0.75,1.00,1.00]$} & {$[0.53,0.71,0.71]$} \\
\hline Very small-maybe & (VS-M) & $\tilde{1}$ & {$[0.00,0.00,0.25]$} & {$[0.00,0.00,0.19]$} \\
\hline Small-maybe & $(\mathrm{S}-\mathrm{M})$ & $\tilde{2}$ & {$[0.00,0.25,0.50]$} & {$[0.00,0.19,0.39]$} \\
\hline Medium-maybe & $(\mathrm{M}-\mathrm{M})$ & $\tilde{3}$ & {$[0.25,0.50,0.75]$} & {$[0.19,0.39,0.58]$} \\
\hline Big-maybe & $(\mathrm{B}-\mathrm{M})$ & $\widetilde{4}$ & {$[0.50,0.75,1.00]$} & {$[0.39,0.58,0.77]$} \\
\hline
\end{tabular}


TABLE 7: Continued.

\begin{tabular}{|c|c|c|c|c|}
\hline Linguistic variable & Notation & Deterministic equivalent & Fuzzy number & Z-number \\
\hline Very big-maybe & (VB-M) & $\widetilde{5}$ & {$[0.75,1.00,1.00]$} & {$[0.58,0.77,0.77]$} \\
\hline Very small-likely & (VS-L) & $\widetilde{1}$ & {$[0.00,0.00,0.25]$} & {$[0.00,0.00,0.22]$} \\
\hline Small-likely & $(\mathrm{S}-\mathrm{L})$ & $\tilde{2}$ & {$[0.00,0.25,0.50]$} & {$[0.00,0.22,0.45]$} \\
\hline Medium-likely & $(\mathrm{M}-\mathrm{L})$ & $\tilde{3}$ & {$[0.25,0.50,0.75]$} & {$[0.22,0.45,0.67]$} \\
\hline Big-likely & $(\mathrm{B}-\mathrm{L})$ & $\tilde{4}$ & {$[0.50,0.75,1.00]$} & {$[0.45,0.67,0.89]$} \\
\hline Very big-likely & (VB-L) & $\widetilde{5}$ & {$[0.75,1.00,1.00]$} & {$[0.67,0.89,0.89]$} \\
\hline Very small-most likely & (VS-ML) & $\tilde{1}$ & {$[0.00,0.00,0.25]$} & {$[0.00,0.00,0.24]$} \\
\hline Small-most likely & $(\mathrm{S}-\mathrm{ML})$ & $\tilde{2}$ & {$[0.00,0.25,0.50]$} & {$[0.00,0.24,0.47]$} \\
\hline Medium-most likely & (M-ML) & $\tilde{3}$ & {$[0.25,0.50,0.75]$} & {$[0.24,0.47,0.71]$} \\
\hline Big-most likely & (B-ML) & $\tilde{4}$ & {$[0.50,0.75,1.00]$} & {$[0.47,0.71,0.95]$} \\
\hline Very big-most likely & (VB-ML) & $\widetilde{5}$ & {$[0.75,1.00,1.00]$} & {$[0.71,0.95,0.95]$} \\
\hline Very small-certainly & (VS-C) & $\tilde{1}$ & {$[0.00,0.00,0.25]$} & {$[0.00,0.00,0.25]$} \\
\hline Small-certainly & $(\mathrm{S}-\mathrm{C})$ & $\tilde{2}$ & {$[0.00,0.25,0.50]$} & {$[0.00,0.25,0.50]$} \\
\hline Medium-certainly & $(\mathrm{M}-\mathrm{C})$ & $\tilde{3}$ & {$[0.25,0.50,0.75]$} & {$[0.25,0.50,0.75]$} \\
\hline Big-certainly & $(\mathrm{B}-\mathrm{C})$ & $\tilde{4}$ & {$[0.50,0.75,1.00]$} & {$[0.50,0.75,1.00]$} \\
\hline Very big-certainly & (VB-C) & $\widetilde{5}$ & {$[0.75,1.00,1.00]$} & {$[0.75,1.00,1.00]$} \\
\hline
\end{tabular}

TABLE 8: Filtering of sustainability criteria.

\begin{tabular}{|c|c|c|c|}
\hline Dimension & Code & Criteria & Description \\
\hline \multirow{4}{*}{ Economic } & $E 1$ & Profit from project implementation & $\begin{array}{l}\text { It is the difference between the income from the project and the } \\
\text { related costs in a given period }\end{array}$ \\
\hline & $E 2$ & Fund return rate & $\begin{array}{c}\text { It is a ratio that calculates the return on investment in the form } \\
\text { of a percentage of the initial cost. In other words, in the form of } \\
\text { the initial cost percentage, it is calculated how profitable this } \\
\text { investment has been }\end{array}$ \\
\hline & E3 & Fund return period & $\begin{array}{l}\text { The time that takes for the initial investment in the project to } \\
\text { match its revenue and return the project costs to the investors }\end{array}$ \\
\hline & E4 & $\begin{array}{l}\text { Existence of basic infrastructures for project } \\
\text { implementation }\end{array}$ & $\begin{array}{l}\text { This includes equipment and facilities, skilled manpower, skills } \\
\text { and knowledge, etc., required to implement the desired project }\end{array}$ \\
\hline \multirow{4}{*}{ Social } & $S 1$ & $\begin{array}{l}\text { The employment rate from the project } \\
\text { implementation }\end{array}$ & $\begin{array}{l}\text { It is the number of manpower that will be employed if the } \\
\text { project is implemented }\end{array}$ \\
\hline & $S 2$ & $\begin{array}{l}\text { Social acceptance and support for project } \\
\text { implementation }\end{array}$ & $\begin{array}{l}\text { The level of satisfaction and appropriate feedback from the } \\
\text { community and the general public for the implementation of } \\
\text { the project }\end{array}$ \\
\hline & $S 3$ & $\begin{array}{l}\text { The willingness of unions, organizations, and } \\
\text { government to participate in the project } \\
\text { implementation }\end{array}$ & $\begin{array}{l}\text { The level of support and acceptance of the government and } \\
\text { other organizations for their participation and cooperation in } \\
\text { the implementation of the project }\end{array}$ \\
\hline & $S 4$ & Employee and personnel satisfaction & $\begin{array}{l}\text { The level of willingness of employees in order to be sufficiently } \\
\text { motivated to implement the project }\end{array}$ \\
\hline \multirow{4}{*}{ Environmental } & G1 & $\begin{array}{l}\text { Production rate of greenhouse gases (derived } \\
\text { from natural energy) in the project }\end{array}$ & $\begin{array}{l}\text { The emission of greenhouse gases such as } \mathrm{CO}_{2} \text { and } \mathrm{SO}_{2} \text { in the } \\
\text { event of a project that pollutes the environment }\end{array}$ \\
\hline & G2 & Use of recyclable consumables in the project & $\begin{array}{l}\text { The amount of recyclable material that can be used in other } \\
\text { projects and be recyclable if the project is completed and put } \\
\text { into operation }\end{array}$ \\
\hline & G3 & Renewable energy consumption in the project & $\begin{array}{l}\text { The amount of renewable energy consumption, such as fossil } \\
\text { fuels, if the project is implemented }\end{array}$ \\
\hline & G4 & $\begin{array}{l}\text { The amount of waste from project } \\
\text { implementation }\end{array}$ & $\begin{array}{l}\text { The amount of waste disposal from the material used in the } \\
\text { project to reduce the damage of the waste from that material to } \\
\text { the environment }\end{array}$ \\
\hline
\end{tabular}

a combination of different MADM methods to select urban renewal projects in Turkey. The findings of these methods are compared with the analytical hierarchy process (AHP) and TOPSIS methods [49]. In a 2019 study, a method based on the fuzzy analytical hierarchy process (FAHP) was proposed for decision-making, with cause-and-effect diagrams for use in quality improvement studies. Resources are supposed to be scarce for improvement, and efforts are being made to make the best use of them [50]. An article in 2019 discusses the selection of IT projects. Due to the inherent complexities and uncertainties in the strategic-operational nature of the process and the existence of both quantitative and qualitative criteria, a hybrid mathematical programming model with fuzzy analytical hierarchy process (FAHP) integration with a fuzzy inference system (FIS) is proposed [51]. The 2019 paper presents a fuzzy multicriteria analysis 


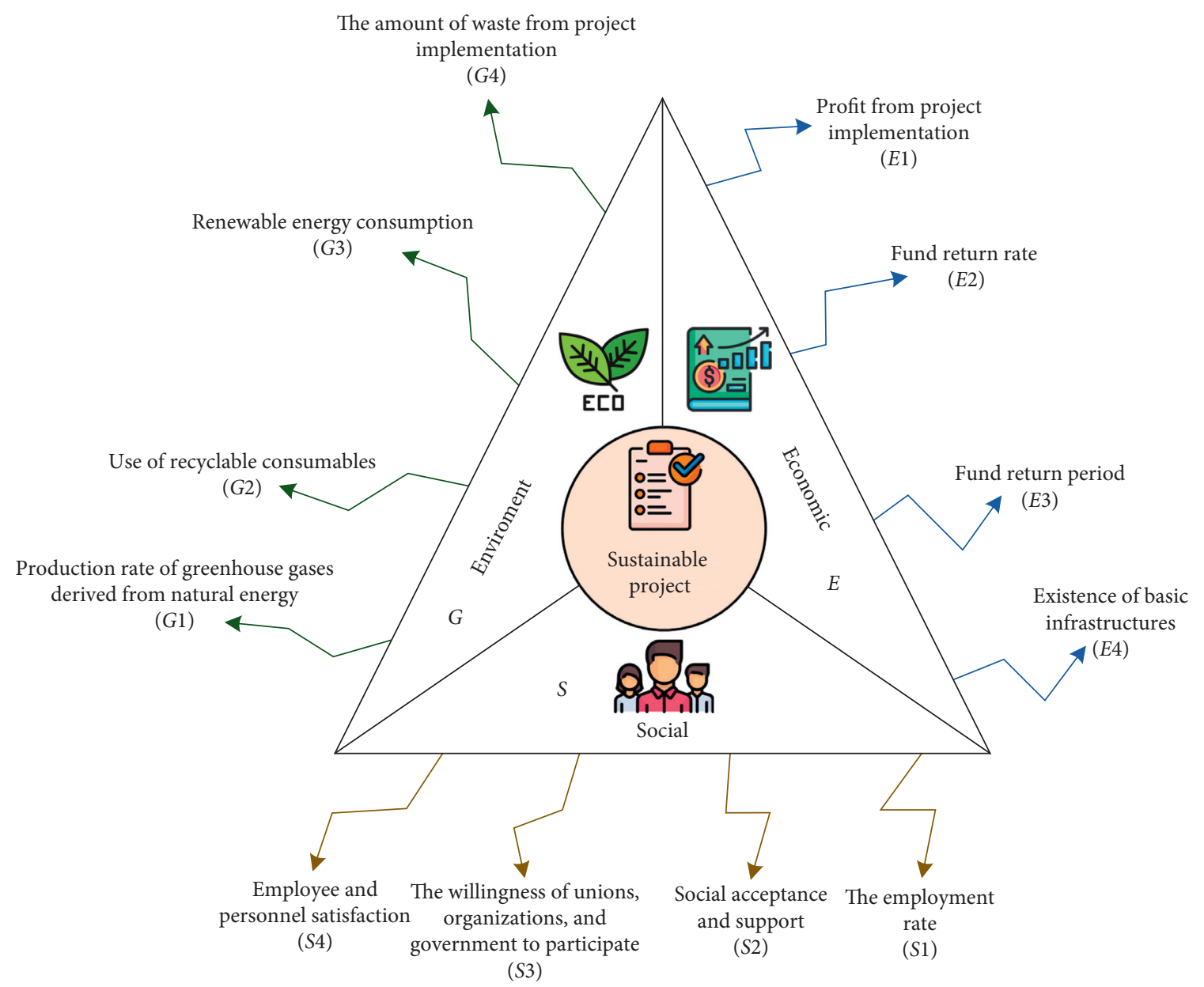

FIgURE 6: Sustainability dimensions and criteria selected in this study.

approach for selecting flood control projects that address economic, social, and environmental aspects. In this study, a fuzzy multicriteria decision-making is proposed to evaluate the project. The fuzzy hierarchical processing method (FAHP) is used to determine weights and criteria. Then, the fuzzy VIKOR method is used to rank the project options [52]. In 2019, an article examines a practical example of project selection to determine the effectiveness of the proposed approach. In this approach, fuzzy AHP is used to analyze the problem while determining the weight of the criteria. Fuzzy TOPSIS is used to rank all selected options and determine the most appropriate project [53]. A 2019 study aims to propose a Euclidean distance-based approximation (EDBA) multicriteria decision-making (MCDM) method for evaluating and selecting a variety of research and development projects [54]. In an article from Brazil in 2019, the ranking of scholarship projects is supported using AHP and PROMETHEE II methods [55]. In an article in 2019, IF-DEMATEL was used to select R\&D projects, primarily to show the relationships between criteria and to eliminate inefficient criteria. Then, using the effective criteria, the most appropriate option is provided using IF-TOPSIS [56]. The 2020 study adopts a three-pillar concept for the assessment of economic, environmental, and social sustainability. The main purpose of this study is to select the target project from the perspective of sustainability in an uncertain decision-making condition [20]. A 2020 study focused on the selection of oil projects using MADM methods in uncertain condition. In the first place, important factors for the selection of oil projects should be collected from previous relevant studies and then filtered using the Delphi method. Oil projects are ranked using a comprehensive approach, including new MADM alternative methods [57]. Table 1 shows a summary of the articles.

According to the review of previous articles, the following research gaps are identified, followed by the innovations of this research:

(i) In previous articles, the combined DEMATELVIKOR approach (DEMATEL for weighting criteria and VIKOR for project ranking) has not been used

(ii) Due to the type of uncertainties used in previous articles, it is observed that most articles have used the fuzzy approach, and the Z-number approach has not been used in the project selection problems

Unlike the traditional weighting method in which the criteria are assumed to be independent, the Z-DEMATEL method is capable of dealing with the existing ambiguities caused by human judgments while also incorporating the 
TABLE 9: Comparison matrix of corresponding numbers to linguistic variable of sustainability criteria in Z-number condition.

\begin{tabular}{|c|c|c|c|c|c|c|c|c|c|c|c|c|}
\hline & $E 1$ & $E 2$ & E3 & $E 4$ & $S 1$ & $S 2$ & S3 & $S 4$ & G1 & G2 & G3 & G4 \\
\hline$E 1$ & $\begin{array}{l}(0.000, \\
0.000, \\
0.000)\end{array}$ & $\begin{array}{l}(0.750, \\
1.000, \\
1.000)\end{array}$ & $\begin{array}{l}(0.000, \\
0.000, \\
0.110)\end{array}$ & $\begin{array}{l}(0.000, \\
0.000, \\
0.240)\end{array}$ & $\begin{array}{l}(0.000, \\
0.250, \\
0.500)\end{array}$ & $\begin{array}{l}(0.000 \\
0.220 \\
0.450)\end{array}$ & $\begin{array}{l}(0.710 \\
0.950, \\
0.950)\end{array}$ & $\begin{array}{l}(0.220 \\
0.450 \\
0.670)\end{array}$ & $\begin{array}{l}(0.000, \\
0.110, \\
0.220)\end{array}$ & $\begin{array}{l}(0.180, \\
0.350, \\
0.530)\end{array}$ & $\begin{array}{c}(0.000 \\
0.180 \\
0.350)\end{array}$ & $\begin{array}{l}(0.110, \\
0.220, \\
0.340)\end{array}$ \\
\hline$E 2$ & $\begin{array}{l}(0.670 \\
0.890 \\
0.890)\end{array}$ & $\begin{array}{l}(0.000 \\
0.000 \\
0.000)\end{array}$ & $\begin{array}{l}(0.000 \\
0.000 \\
0.180)\end{array}$ & $\begin{array}{l}(0.000 \\
0.000 \\
0.220)\end{array}$ & $\begin{array}{l}(0.000, \\
0.180, \\
0.350)\end{array}$ & $\begin{array}{c}(0.000 \\
0.180 \\
0.350)\end{array}$ & $\begin{array}{l}(0.470, \\
0.630 \\
0.630)\end{array}$ & $\begin{array}{l}(0.240 \\
0.470 \\
0.710)\end{array}$ & $\begin{array}{c}(0.000 \\
0.240 \\
0.470)\end{array}$ & $\begin{array}{l}(0.250 \\
0.500 \\
0.750)\end{array}$ & $\begin{array}{c}(0.000, \\
0.190, \\
0.390)\end{array}$ & $\begin{array}{c}(0.190, \\
0.390, \\
0.580)\end{array}$ \\
\hline E3 & $\begin{array}{l}(0.0 \\
0.00 \\
0.19\end{array}$ & $\begin{array}{l}(0.00 \\
0.00 \\
0.19\end{array}$ & $\begin{array}{l}(0.000, \\
0.000, \\
0.000)\end{array}$ & $\begin{array}{l}(0.000, \\
0.000, \\
0.110)\end{array}$ & $\begin{array}{c}(0.000, \\
0.250, \\
0.500)\end{array}$ & $\begin{array}{l}(0.000 \\
0.000 \\
0.220)\end{array}$ & $\begin{array}{l}(0.530 \\
0.710 \\
0.710)\end{array}$ & $\begin{array}{l}(0.250 \\
0.500 \\
0.750)\end{array}$ & $\begin{array}{l}(0.000 \\
0.000 \\
0.160)\end{array}$ & $\begin{array}{l}(0.000 \\
0.000 \\
0.250)\end{array}$ & $\begin{array}{l}(0.000, \\
0.000, \\
0.110)\end{array}$ & $\begin{array}{c}(0.000 \\
0.000 \\
0.220)\end{array}$ \\
\hline$E 4$ & $\begin{array}{l}(0.190, \\
0.390, \\
0.580)\end{array}$ & $\begin{array}{l}(0.000, \\
0.240, \\
0.470)\end{array}$ & $\begin{array}{c}(0.000, \\
0.220, \\
0.450)\end{array}$ & $\begin{array}{l}(0.000, \\
0.000, \\
0.000)\end{array}$ & $\begin{array}{l}(0.180, \\
0.350, \\
0.530)\end{array}$ & $\begin{array}{l}(0.000, \\
0.000, \\
0.160)\end{array}$ & $\begin{array}{l}(0.450, \\
0.670, \\
0.890)\end{array}$ & $\begin{array}{l}(0.240, \\
0.470, \\
0.710)\end{array}$ & $\begin{array}{c}(0.390, \\
0.580, \\
0.770)\end{array}$ & $\begin{array}{l}(0.500, \\
0.750, \\
1.000)\end{array}$ & $\begin{array}{l}(0.240, \\
0.470, \\
0.710)\end{array}$ & $\begin{array}{l}(0.220 \\
0.450 \\
0.670)\end{array}$ \\
\hline$S 1$ & $\begin{array}{l}(0.000, \\
0.000, \\
0.240)\end{array}$ & $\begin{array}{l}(0.000, \\
0.000, \\
0.250)\end{array}$ & $\begin{array}{c}(0.000, \\
0.000, \\
0.160)\end{array}$ & $\begin{array}{l}(0.000, \\
0.000, \\
0.180)\end{array}$ & $\begin{array}{l}(0.000, \\
0.000, \\
0.000)\end{array}$ & $\begin{array}{l}(0.340, \\
0.450, \\
0.450)\end{array}$ & $\begin{array}{l}(0.220, \\
0.340, \\
0.450)\end{array}$ & $\begin{array}{l}(0.320, \\
0.470, \\
0.630)\end{array}$ & $\begin{array}{l}(0.000, \\
0.000, \\
0.220)\end{array}$ & $\begin{array}{l}(0.000, \\
0.000, \\
0.220)\end{array}$ & $\begin{array}{l}(0.000, \\
0.000, \\
0.110)\end{array}$ & $\begin{array}{c}(0.000 \\
0.000 \\
0.180)\end{array}$ \\
\hline$S 2$ & $\begin{array}{c}(0.000, \\
0.180, \\
0.350)\end{array}$ & $\begin{array}{l}(0.0 \\
0.00 \\
0.18\end{array}$ & $\begin{array}{l}(0.000, \\
0.000, \\
0.240)\end{array}$ & $\begin{array}{l}(0.000 \\
0.000 \\
0.190)\end{array}$ & $\begin{array}{c}(0.000, \\
0.240, \\
0.470)\end{array}$ & $\begin{array}{l}(0.000 \\
0.000 \\
0.000)\end{array}$ & $\begin{array}{l}(0.670, \\
0.890, \\
0.890)\end{array}$ & $\begin{array}{l}(0.350 \\
0.530 \\
0.710)\end{array}$ & $\begin{array}{c}(0.000, \\
0.000, \\
0.180)\end{array}$ & $\begin{array}{l}(0.000, \\
0.000, \\
0.250)\end{array}$ & $\begin{array}{l}(0.000, \\
0.000, \\
0.240)\end{array}$ & $\begin{array}{c}(0.000 \\
0.000 \\
0.180)\end{array}$ \\
\hline S3 & & $\begin{array}{l}(0.110, \\
0.220 \\
0.340)\end{array}$ & $\begin{array}{l}(0.320, \\
0.470, \\
0.630)\end{array}$ & $\begin{array}{l}(0.000 \\
0.000 \\
0.190)\end{array}$ & $\begin{array}{l}(0.320, \\
0.470, \\
0.630)\end{array}$ & $\begin{array}{l}(0.350, \\
0.530 \\
0.710)\end{array}$ & $\begin{array}{l}(0.000, \\
0.000, \\
0.000)\end{array}$ & $\begin{array}{l}(0.320, \\
0.470, \\
0.630)\end{array}$ & $\begin{array}{l}(0.500, \\
0.750, \\
1.000)\end{array}$ & $\begin{array}{l}(0.180, \\
0.350, \\
0.530)\end{array}$ & $\begin{array}{l}(0.390, \\
0.580, \\
0.770)\end{array}$ & $\begin{array}{c}(0.190 \\
0.390 \\
0.580)\end{array}$ \\
\hline$S 4$ & $\begin{array}{c}(0.000, \\
0.180, \\
0.350)\end{array}$ & $\begin{array}{l}(0.000, \\
0.190, \\
0.390)\end{array}$ & $\begin{array}{l}(0.000, \\
0.000, \\
0.220)\end{array}$ & $\begin{array}{l}(0.000 \\
0.000 \\
0.180)\end{array}$ & $\begin{array}{l}(0.000 \\
0.190, \\
0.390)\end{array}$ & $\begin{array}{c}(0.000, \\
0.180, \\
0.350)\end{array}$ & $\begin{array}{l}(0.180, \\
0.350, \\
0.530)\end{array}$ & $\begin{array}{l}(0.000, \\
0.000, \\
0.000)\end{array}$ & $\begin{array}{l}(0.000, \\
0.000, \\
0.190)\end{array}$ & $\begin{array}{l}(0.000 \\
0.000 \\
0.240)\end{array}$ & $\begin{array}{l}(0.000, \\
0.000, \\
0.220)\end{array}$ & $\begin{array}{c}(0.000 \\
0.000, \\
0.160)\end{array}$ \\
\hline$G 1$ & $\begin{array}{c}(0.000, \\
0.190, \\
0.390)\end{array}$ & $\begin{array}{l}(0.000, \\
0.000, \\
0.220)\end{array}$ & $\begin{array}{l}(0.000, \\
0.000, \\
0.250)\end{array}$ & $\begin{array}{l}(0.000, \\
0.000, \\
0.240)\end{array}$ & $\begin{array}{c}(0.000, \\
0.190, \\
0.390)\end{array}$ & $\begin{array}{c}(0.470, \\
0.710, \\
0.950)\end{array}$ & $\begin{array}{l}(0.500, \\
0.750, \\
1.000)\end{array}$ & $\begin{array}{l}(0.000, \\
0.000, \\
0.240)\end{array}$ & $\begin{array}{c}(0.000, \\
0.000, \\
0.000)\end{array}$ & $\begin{array}{c}(0.000, \\
0.160, \\
0.320)\end{array}$ & $\begin{array}{c}(0.000, \\
0.250, \\
0.500)\end{array}$ & $\begin{array}{c}(0.000 \\
0.110 \\
0.220)\end{array}$ \\
\hline G2 & $\begin{array}{c}(0.220, \\
0.450, \\
0.670)\end{array}$ & $\begin{array}{l}(0.220, \\
0.450, \\
0.670)\end{array}$ & $\begin{array}{l}(0.000 \\
0.000 \\
0.190)\end{array}$ & $\begin{array}{l}(0.000, \\
0.240, \\
0.470)\end{array}$ & $\begin{array}{c}(0.000, \\
0.000, \\
0.220)\end{array}$ & $\begin{array}{l}(0.750, \\
1.000, \\
1.000)\end{array}$ & $\begin{array}{l}(0.470, \\
0.630, \\
0.630)\end{array}$ & $\begin{array}{l}(0.160 \\
0.320 \\
0.470)\end{array}$ & $\begin{array}{c}(0.470, \\
0.710, \\
0.950)\end{array}$ & $\begin{array}{l}(0.000, \\
0.000, \\
0.000)\end{array}$ & $\begin{array}{l}(0.160, \\
0.320, \\
0.470)\end{array}$ & $\begin{array}{c}(0.190 \\
0.390 \\
0.580)\end{array}$ \\
\hline G3 & $\begin{array}{l}(0.250, \\
0.500, \\
0.750)\end{array}$ & $\begin{array}{l}(0.110, \\
0.220, \\
0.340)\end{array}$ & $\begin{array}{c}(0.000, \\
0.000, \\
0.250)\end{array}$ & $\begin{array}{l}(0.000, \\
0.000, \\
0.250)\end{array}$ & $\begin{array}{l}(0.000, \\
0.000, \\
0.110)\end{array}$ & $\begin{array}{l}(0.470, \\
0.630, \\
0.630)\end{array}$ & $\begin{array}{c}(0.710 \\
0.950 \\
0.950)\end{array}$ & $\begin{array}{l}(0.180, \\
0.350, \\
0.530)\end{array}$ & $\begin{array}{l}(0.250, \\
0.500, \\
0.750)\end{array}$ & $\begin{array}{l}(0.000, \\
0.000, \\
0.110)\end{array}$ & $\begin{array}{c}(0.000, \\
0.000, \\
0.000)\end{array}$ & $\begin{array}{c}(0.000 \\
0.000 \\
0.220)\end{array}$ \\
\hline G4 & $\begin{array}{c}(0.000, \\
0.180, \\
0.350)\end{array}$ & $\begin{array}{l}(0.000, \\
0.000, \\
0.190)\end{array}$ & $\begin{array}{c}(0.000, \\
0.000, \\
0.250)\end{array}$ & $\begin{array}{l}(0.000, \\
0.000, \\
0.220)\end{array}$ & $\begin{array}{c}(0.000, \\
0.000, \\
0.180)\end{array}$ & $\begin{array}{l}(0.390, \\
0.580, \\
0.770)\end{array}$ & $\begin{array}{l}(0.220, \\
0.340, \\
0.450)\end{array}$ & $\begin{array}{l}(0.000, \\
0.250, \\
0.500)\end{array}$ & $\begin{array}{c}(0.000, \\
0.000, \\
0.250)\end{array}$ & $\begin{array}{l}(0.000, \\
0.000, \\
0.190)\end{array}$ & $\begin{array}{c}(0.000, \\
0.000, \\
0.220)\end{array}$ & $\begin{array}{c}(0.000 \\
0.000 \\
0.000)\end{array}$ \\
\hline
\end{tabular}

complicated interactive relationships among the sustainable criteria. Z-VIKOR, which is capable of tackling data uncertainties, has also been suggested so as to rank sustainable project portfolio under uncertain conditions. By and large, the Z-number multicriteria decision-making method proposed for prioritizing the alternative project portfolio has the following advantages:

(1) Using Z-DEMATEL to specify the weights of the sustainable criteria, independent relationships between the criteria can be taken into account

(2) Any ambiguity and obscurity caused by human judgments can be effectively dealt with after the weights of the sustainable criteria are determined

(3) The method is able to carry out group decisionmaking when ranking the alternative project portfolio given that the opinions of experts can be considered simultaneously

(4) Uncertainties can be taken into account via the proposed Z-VIKOR method when ranking the alternative project portfolio
(5) Given that Z-number considers probabilities and reliability of fuzzy numbers, results obtained from Znumber are closer to real-world assumptions, leading to more reliable results in terms of data reliability

2.4. DEMATEL and VIKOR Approach Application. A systematic FMEA has been developed by Liu et al. in 2019 in which a hybrid multiple criteria decision-making approach (AHP-DEMATEL) has been utilized to help improve risk assessment of the traditional FMEA, taking the interrelations of failure modes into consideration. A comparative numerical study has been carried out to validate their model [58]. Xu et al. have investigated barriers to the development of hydrogen refuelling stations (HRSs) in China using a DEMATEL method that first identifies a list of barriers via a literature survey, followed by implementing a fuzzy DEMATEL approach to prioritize the barriers. As a result, the main barriers have been identified, after which some policy measures have been suggested to alleviate the problem [59]. In 2019, Jiang et al. presented a large-group evaluation approach by means of linguistic Z-numbers and 
TABLE 10: Normalized matrix of sustainability criteria in Z-number condition.

\begin{tabular}{|c|c|c|c|c|c|c|c|c|c|c|c|c|}
\hline & $E 1$ & $E 2$ & E3 & $E 4$ & $S 1$ & $S 2$ & S3 & $S 4$ & $G 1$ & G2 & G3 & G4 \\
\hline$E 1$ & $\begin{array}{l}(0.000, \\
0.000, \\
0.000)\end{array}$ & $\begin{array}{l}(0.108, \\
0.144, \\
0.144)\end{array}$ & $\begin{array}{l}(0.000 \\
0.000 \\
0.016)\end{array}$ & $\begin{array}{l}(0.000 \\
0.000 \\
0.035)\end{array}$ & $\begin{array}{l}(0.000, \\
0.036, \\
0.072)\end{array}$ & $\begin{array}{l}(0.000, \\
0.032, \\
0.065)\end{array}$ & $\begin{array}{l}(0.102, \\
0.137 \\
0.137)\end{array}$ & $\begin{array}{l}(0.032, \\
0.065 \\
0.097)\end{array}$ & $\begin{array}{c}(0.000, \\
0.016, \\
0.032)\end{array}$ & $\begin{array}{l}(0.026 \\
0.050 \\
0.076)\end{array}$ & $\begin{array}{l}(0.000 \\
0.026 \\
0.050)\end{array}$ & $\begin{array}{l}(0.016 \\
0.032, \\
0.049)\end{array}$ \\
\hline$E 2$ & $\begin{array}{c}(0.097 \\
0.128 \\
0.128)\end{array}$ & $\begin{array}{l}(0.000 \\
0.000 \\
0.000)\end{array}$ & $\begin{array}{c}(0.000, \\
0.000, \\
0.026)\end{array}$ & $\begin{array}{l}(0.000 \\
0.000 \\
0.032)\end{array}$ & $\begin{array}{l}(0.000 \\
0.026 \\
0.050)\end{array}$ & $\begin{array}{l}(0.000 \\
0.026 \\
0.050)\end{array}$ & $\begin{array}{l}(0.068, \\
0.091 \\
0.091)\end{array}$ & $\begin{array}{l}(0.035 \\
0.068 \\
0.102)\end{array}$ & $\begin{array}{c}(0.000 \\
0.035 \\
0.068)\end{array}$ & $\begin{array}{l}(0.036 \\
0.072, \\
0.108)\end{array}$ & $\begin{array}{l}(0.000, \\
0.027, \\
0.056)\end{array}$ & $\begin{array}{l}(0.027 \\
0.056, \\
0.084)\end{array}$ \\
\hline E3 & $\begin{array}{l}(0.00 \\
0.00 \\
0.02\end{array}$ & $\begin{array}{l}(0.000 \\
0.000 \\
0.027)\end{array}$ & $\begin{array}{l}(0.000 \\
0.000 \\
0.000)\end{array}$ & $\begin{array}{l}(0.000 \\
0.000 \\
0.016)\end{array}$ & $\begin{array}{l}(0.000 \\
0.036 \\
0.072)\end{array}$ & $\begin{array}{l}(0.000, \\
0.000 \\
0.032)\end{array}$ & $\begin{array}{l}(0.076, \\
0.102, \\
0.102)\end{array}$ & $\begin{array}{l}(0.036 \\
0.072, \\
0.108)\end{array}$ & $\begin{array}{l}(0.000 \\
0.000 \\
0.023)\end{array}$ & $\begin{array}{l}(0.000 \\
0.000 \\
0.036)\end{array}$ & $\begin{array}{l}(0.000 \\
0.000 \\
0.016)\end{array}$ & $\begin{array}{l}(0.000, \\
0.000, \\
0.032)\end{array}$ \\
\hline$E 4$ & $\begin{array}{c}(0.027, \\
0.056, \\
0.084)\end{array}$ & $\begin{array}{l}(0.000, \\
0.035, \\
0.068)\end{array}$ & $\begin{array}{c}(0.000, \\
0.032, \\
0.065)\end{array}$ & $\begin{array}{l}(0.000, \\
0.000, \\
0.000)\end{array}$ & $\begin{array}{c}(0.026, \\
0.050, \\
0.076)\end{array}$ & $\begin{array}{l}(0.000, \\
0.000 \\
0.023)\end{array}$ & $\begin{array}{l}(0.065, \\
0.097 \\
0.128)\end{array}$ & $\begin{array}{l}(0.035 \\
0.068 \\
0.102)\end{array}$ & $\begin{array}{l}(0.056 \\
0.084, \\
0.111)\end{array}$ & $\begin{array}{l}(0.072, \\
0.108, \\
0.144)\end{array}$ & $\begin{array}{l}(0.035, \\
0.068, \\
0.102)\end{array}$ & $\begin{array}{l}(0.032, \\
0.065, \\
0.097)\end{array}$ \\
\hline$S 1$ & $\begin{array}{l}(0.000, \\
0.000, \\
0.035)\end{array}$ & $\begin{array}{l}(0.000, \\
0.000, \\
0.036)\end{array}$ & $\begin{array}{c}(0.000, \\
0.000 \\
0.023)\end{array}$ & $\begin{array}{l}(0.000, \\
0.000, \\
0.026)\end{array}$ & $\begin{array}{c}(0.000, \\
0.000, \\
0.000)\end{array}$ & $\begin{array}{l}(0.049 \\
0.065 \\
0.065)\end{array}$ & $\begin{array}{l}(0.032, \\
0.049 \\
0.065)\end{array}$ & $\begin{array}{l}(0.046 \\
0.068 \\
0.091)\end{array}$ & $\begin{array}{c}(0.000, \\
0.000, \\
0.032)\end{array}$ & $\begin{array}{l}(0.000, \\
0.000, \\
0.032)\end{array}$ & $\begin{array}{c}(0.000, \\
0.000, \\
0.016)\end{array}$ & $\begin{array}{l}(0.000, \\
0.000, \\
0.026)\end{array}$ \\
\hline$S 2$ & $\begin{array}{l}(0.00 \\
0.02 \\
0.05\end{array}$ & $\begin{array}{l}(0.000 \\
0.000 \\
0.026)\end{array}$ & $\begin{array}{l}(0.00 \\
0.00 \\
0.03\end{array}$ & $\begin{array}{l}(0.000 \\
0.000 \\
0.027)\end{array}$ & $\begin{array}{l}(0.000, \\
0.035 \\
0.068)\end{array}$ & $\begin{array}{l}(0.000, \\
0.000, \\
0.000)\end{array}$ & $\begin{array}{c}(0.097 \\
0.128 \\
0.128)\end{array}$ & $\begin{array}{l}(0.050, \\
0.076, \\
0.102)\end{array}$ & $\begin{array}{l}(0.000, \\
0.000, \\
0.026)\end{array}$ & $\begin{array}{l}(0.000, \\
0.000, \\
0.036)\end{array}$ & $\begin{array}{l}(0.000, \\
0.000, \\
0.035)\end{array}$ & $\begin{array}{l}(0.000, \\
0.000, \\
0.026)\end{array}$ \\
\hline S3 & & $\begin{array}{l}(0.016 \\
0.032 \\
0.049\end{array}$ & $\begin{array}{l}(0.046 \\
0.068 \\
0.091)\end{array}$ & $\begin{array}{l}(0.000 \\
0.000 \\
0.027)\end{array}$ & $\begin{array}{c}(0.046 \\
0.068 \\
0.091)\end{array}$ & $\begin{array}{l}(0.050, \\
0.076, \\
0.102)\end{array}$ & $\begin{array}{l}(0.000, \\
0.000, \\
0.000)\end{array}$ & $\begin{array}{l}(0.046 \\
0.068 \\
0.091)\end{array}$ & $\begin{array}{l}(0.072, \\
0.108, \\
0.144)\end{array}$ & $\begin{array}{l}(0.026, \\
0.050 \\
0.076)\end{array}$ & $\begin{array}{l}(0.056, \\
0.084, \\
0.111)\end{array}$ & $\begin{array}{l}(0.027, \\
0.056, \\
0.084)\end{array}$ \\
\hline$S 4$ & $\begin{array}{l}(0.000, \\
0.026, \\
0.050)\end{array}$ & $\begin{array}{l}(0.000 \\
0.027 \\
0.056)\end{array}$ & $\begin{array}{l}(0.000, \\
0.000, \\
0.032)\end{array}$ & $\begin{array}{l}(0.000 \\
0.000 \\
0.026)\end{array}$ & $\begin{array}{l}(0.000 \\
0.027 \\
0.056)\end{array}$ & $\begin{array}{l}(0.000, \\
0.026, \\
0.050)\end{array}$ & $\begin{array}{l}(0.026 \\
0.050 \\
0.076)\end{array}$ & $\begin{array}{l}(0.000, \\
0.000, \\
0.000)\end{array}$ & $\begin{array}{l}(0.000 \\
0.000 \\
0.027)\end{array}$ & $\begin{array}{l}(0.000 \\
0.000 \\
0.035)\end{array}$ & $\begin{array}{l}(0.000, \\
0.000, \\
0.032)\end{array}$ & $\begin{array}{l}(0.000, \\
0.000, \\
0.023)\end{array}$ \\
\hline$G 1$ & $\begin{array}{c}(0.000, \\
0.027, \\
0.056)\end{array}$ & $\begin{array}{l}(0.000, \\
0.000, \\
0.032)\end{array}$ & $\begin{array}{l}(0.000, \\
0.000 \\
0.036)\end{array}$ & $\begin{array}{l}(0.000, \\
0.000, \\
0.035)\end{array}$ & $\begin{array}{c}(0.000, \\
0.027, \\
0.056)\end{array}$ & $\begin{array}{l}(0.068, \\
0.102, \\
0.137)\end{array}$ & $\begin{array}{l}(0.072, \\
0.108, \\
0.144)\end{array}$ & $\begin{array}{l}(0.000, \\
0.000, \\
0.035)\end{array}$ & $\begin{array}{c}(0.000, \\
0.000, \\
0.000)\end{array}$ & $\begin{array}{l}(0.000 \\
0.023, \\
0.046)\end{array}$ & $\begin{array}{c}(0.000, \\
0.036, \\
0.072)\end{array}$ & $\begin{array}{c}(0.000, \\
0.016, \\
0.032)\end{array}$ \\
\hline G2 & $\begin{array}{l}(0.032, \\
0.065, \\
0.097)\end{array}$ & $\begin{array}{l}(0.032, \\
0.065, \\
0.097)\end{array}$ & $\begin{array}{l}(0.000 \\
0.000 \\
0.027)\end{array}$ & $\begin{array}{l}(0.000, \\
0.035, \\
0.068)\end{array}$ & $\begin{array}{c}(0.000, \\
0.000, \\
0.032)\end{array}$ & $\begin{array}{l}(0.108, \\
0.144, \\
0.144)\end{array}$ & $\begin{array}{c}(0.068, \\
0.091 \\
0.091)\end{array}$ & $\begin{array}{l}(0.023, \\
0.046, \\
0.068)\end{array}$ & $\begin{array}{c}(0.068, \\
0.102, \\
0.137)\end{array}$ & $\begin{array}{l}(0.000, \\
0.000, \\
0.000)\end{array}$ & $\begin{array}{c}(0.023, \\
0.046, \\
0.068)\end{array}$ & $\begin{array}{c}(0.027, \\
0.056, \\
0.084)\end{array}$ \\
\hline G3 & $\begin{array}{l}(0.036, \\
0.072, \\
0.108)\end{array}$ & $\begin{array}{l}(0.016 \\
0.032, \\
0.049)\end{array}$ & $\begin{array}{c}(0.000, \\
0.000, \\
0.036)\end{array}$ & $\begin{array}{l}(0.000 \\
0.000 \\
0.036)\end{array}$ & $\begin{array}{c}(0.000, \\
0.000, \\
0.016)\end{array}$ & $\begin{array}{l}(0.068, \\
0.091, \\
0.091)\end{array}$ & $\begin{array}{l}(0.102, \\
0.137 \\
0.137)\end{array}$ & $\begin{array}{l}(0.026, \\
0.050, \\
0.076)\end{array}$ & $\begin{array}{l}(0.036, \\
0.072, \\
0.108)\end{array}$ & $\begin{array}{l}(0.000, \\
0.000, \\
0.016)\end{array}$ & $\begin{array}{l}(0.000, \\
0.000, \\
0.000)\end{array}$ & $\begin{array}{c}(0.000, \\
0.000, \\
0.032)\end{array}$ \\
\hline G4 & $\begin{array}{l}(0.000, \\
0.026, \\
0.050)\end{array}$ & $\begin{array}{l}(0.000, \\
0.000 \\
0.027)\end{array}$ & $\begin{array}{l}(0.000, \\
0.000, \\
0.036)\end{array}$ & $\begin{array}{l}(0.000, \\
0.000, \\
0.032)\end{array}$ & $\begin{array}{l}(0.000, \\
0.000, \\
0.026)\end{array}$ & $\begin{array}{l}(0.056 \\
0.084, \\
0.111)\end{array}$ & $\begin{array}{l}(0.032, \\
0.049 \\
0.065)\end{array}$ & $\begin{array}{l}(0.000, \\
0.036, \\
0.072)\end{array}$ & $\begin{array}{c}(0.000, \\
0.000, \\
0.036)\end{array}$ & $\begin{array}{l}(0.000, \\
0.000, \\
0.027)\end{array}$ & $\begin{array}{l}(0.000, \\
0.000, \\
0.032)\end{array}$ & $\begin{array}{l}(0.000, \\
0.000, \\
0.000)\end{array}$ \\
\hline
\end{tabular}

TABLE 11: Total relationship matrix of sustainability criteria in Z-number condition.

\begin{tabular}{|c|c|c|c|c|c|c|c|c|c|c|c|c|}
\hline & $E 1$ & $E 2$ & E3 & $E 4$ & $S 1$ & $S 2$ & S3 & $S 4$ & $G 1$ & G2 & G3 & G4 \\
\hline$E 1$ & $\begin{array}{l}(0.015 \\
0.048, \\
0.165)\end{array}$ & $\begin{array}{c}(0.113, \\
0.167, \\
0.266)\end{array}$ & $\begin{array}{l}(0.005 \\
0.014 \\
0.114)\end{array}$ & $\begin{array}{l}(0.000 \\
0.003 \\
0.113)\end{array}$ & $\begin{array}{l}(0.005, \\
0.064, \\
0.205)\end{array}$ & $\begin{array}{l}(0.012, \\
0.087 \\
0.253)\end{array}$ & $\begin{array}{l}(0.119, \\
0.204, \\
0.361)\end{array}$ & $\begin{array}{c}(0.044, \\
0.114, \\
0.288)\end{array}$ & $\begin{array}{l}(0.011, \\
0.056 \\
0.197)\end{array}$ & $\begin{array}{l}(0.033, \\
0.077 \\
0.208)\end{array}$ & $\begin{array}{l}(0.007 \\
0.055 \\
0.182)\end{array}$ & $\begin{array}{c}(0.023, \\
0.059, \\
0.172)\end{array}$ \\
\hline$E 2$ & $\begin{array}{l}(0.101, \\
0.160, \\
0.281)\end{array}$ & $\begin{array}{l}(0.014, \\
0.039, \\
0.142)\end{array}$ & $\begin{array}{l}(0.004, \\
0.011, \\
0.122)\end{array}$ & $\begin{array}{l}(0.000 \\
0.003, \\
0.113)\end{array}$ & $\begin{array}{l}(0.004, \\
0.052, \\
0.187)\end{array}$ & $\begin{array}{l}(0.012 \\
0.083 \\
0.248)\end{array}$ & $\begin{array}{c}(0.087 \\
0.164, \\
0.329)\end{array}$ & $\begin{array}{l}(0.044, \\
0.113 \\
0.294)\end{array}$ & $\begin{array}{l}(0.009, \\
0.070, \\
0.229)\end{array}$ & $\begin{array}{l}(0.041 \\
0.093 \\
0.235)\end{array}$ & $\begin{array}{l}(0.006 \\
0.053 \\
0.188)\end{array}$ & $\begin{array}{c}(0.033, \\
0.079, \\
0.202)\end{array}$ \\
\hline E3 & $\begin{array}{l}(0.002 \\
0.011, \\
0.121)\end{array}$ & $\begin{array}{l}(0.002, \\
0.009, \\
0.106)\end{array}$ & $\begin{array}{l}(0.004, \\
0.008 \\
0.060)\end{array}$ & $\begin{array}{l}(0.000 \\
0.000 \\
0.063)\end{array}$ & $\begin{array}{l}(0.004, \\
0.049, \\
0.154)\end{array}$ & $\begin{array}{c}(0.005 \\
0.019 \\
0.147)\end{array}$ & $\begin{array}{c}(0.080 \\
0.119, \\
0.236)\end{array}$ & $\begin{array}{l}(0.041, \\
0.088, \\
0.221)\end{array}$ & $\begin{array}{l}(0.006 \\
0.015 \\
0.121)\end{array}$ & $\begin{array}{l}(0.002 \\
0.008 \\
0.114)\end{array}$ & $\begin{array}{c}(0.005 \\
0.011 \\
0.096)\end{array}$ & $\begin{array}{l}(0.002, \\
0.008, \\
0.104)\end{array}$ \\
\hline$E 4$ & $\begin{array}{c}(0.034, \\
0.101, \\
0.283)\end{array}$ & $\begin{array}{l}(0.008, \\
0.070, \\
0.235)\end{array}$ & $\begin{array}{l}(0.004 \\
0.044 \\
0.184)\end{array}$ & $\begin{array}{l}(0.000 \\
0.005, \\
0.103)\end{array}$ & $\begin{array}{l}(0.030 \\
0.080 \\
0.244)\end{array}$ & $\begin{array}{l}(0.024, \\
0.076 \\
0.276)\end{array}$ & $\begin{array}{c}(0.089, \\
0.185, \\
0.423)\end{array}$ & $\begin{array}{c}(0.046, \\
0.121, \\
0.342)\end{array}$ & $\begin{array}{c}(0.069 \\
0.128 \\
0.315)\end{array}$ & $\begin{array}{c}(0.076, \\
0.131, \\
0.300)\end{array}$ & $\begin{array}{l}(0.041 \\
0.099 \\
0.265)\end{array}$ & $\begin{array}{c}(0.037 \\
0.092 \\
0.246)\end{array}$ \\
\hline$S 1$ & $\begin{array}{l}(0.001 \\
0.009 \\
0.122)\end{array}$ & $\begin{array}{l}(0.001, \\
0.006, \\
0.109)\end{array}$ & $\begin{array}{l}(0.002 \\
0.005 \\
0.078)\end{array}$ & $\begin{array}{l}(0.000 \\
0.000 \\
0.069)\end{array}$ & $\begin{array}{c}(0.002, \\
0.010 \\
0.079)\end{array}$ & $\begin{array}{l}(0.052, \\
0.076, \\
0.167)\end{array}$ & $\begin{array}{l}(0.039 \\
0.068 \\
0.195)\end{array}$ & $\begin{array}{l}(0.051, \\
0.081, \\
0.196)\end{array}$ & $\begin{array}{l}(0.003, \\
0.009 \\
0.120)\end{array}$ & $\begin{array}{l}(0.001 \\
0.005 \\
0.105)\end{array}$ & $\begin{array}{l}(0.002, \\
0.007 \\
0.090)\end{array}$ & $\begin{array}{c}(0.001 \\
0.005 \\
0.093)\end{array}$ \\
\hline$S 2$ & $\begin{array}{c}(0.003 \\
0.041 \\
0.159)\end{array}$ & $\begin{array}{l}(0.002, \\
0.015, \\
0.120)\end{array}$ & $\begin{array}{l}(0.005 \\
0.010 \\
0.105)\end{array}$ & $\begin{array}{l}(0.000 \\
0.000 \\
0.082)\end{array}$ & $\begin{array}{c}(0.005, \\
0.051, \\
0.164)\end{array}$ & $\begin{array}{l}(0.007 \\
0.025 \\
0.135)\end{array}$ & $\begin{array}{l}(0.101 \\
0.153 \\
0.285)\end{array}$ & $\begin{array}{l}(0.056 \\
0.098 \\
0.236)\end{array}$ & $\begin{array}{l}(0.008, \\
0.020 \\
0.142)\end{array}$ & $\begin{array}{c}(0.003, \\
0.011, \\
0.128)\end{array}$ & $\begin{array}{c}(0.006 \\
0.016 \\
0.128)\end{array}$ & $\begin{array}{c}(0.003, \\
0.012, \\
0.111)\end{array}$ \\
\hline$S 3$ & $\begin{array}{l}(0.029, \\
0.082, \\
0.240)\end{array}$ & $\begin{array}{l}(0.021, \\
0.057, \\
0.192)\end{array}$ & $\begin{array}{l}(0.048, \\
0.074, \\
0.191)\end{array}$ & $\begin{array}{l}(0.000 \\
0.002, \\
0.116)\end{array}$ & $\begin{array}{l}(0.048 \\
0.093 \\
0.237)\end{array}$ & $\begin{array}{l}(0.068, \\
0.135, \\
0.314)\end{array}$ & $\begin{array}{l}(0.032, \\
0.090 \\
0.275)\end{array}$ & $\begin{array}{l}(0.059, \\
0.116, \\
0.304)\end{array}$ & $\begin{array}{c}(0.078, \\
0.136, \\
0.309)\end{array}$ & $\begin{array}{l}(0.028, \\
0.067 \\
0.213)\end{array}$ & $\begin{array}{c}(0.059 \\
0.103 \\
0.247)\end{array}$ & $\begin{array}{l}(0.030, \\
0.073, \\
0.208)\end{array}$ \\
\hline
\end{tabular}


TABle 11: Continued.

\begin{tabular}{ccccccccccccccc}
\hline & $E 1$ & $E 2$ & $E 3$ & $E 4$ & $S 1$ & $S 2$ & $S 3$ & $S 4$ & $G 1$ & $G 2$ & $G 3$ \\
\hline \multirow{4}{*}{$S 4$} & $(0.001$, & $(0.001$, & $(0.001$, & $(0.000$, & $(0.001$, & $(0.002$, & $(0.027$, & $(0.002$, & $(0.002$, & $(0.001$, & $(0.002$, & $(0.001$, \\
& 0.037, & 0.036, & 0.005, & 0.000, & 0.037, & 0.040, & 0.071, & 0.017, & 0.011, & 0.008, & 0.009, & 0.008, \\
& $0.145)$ & $0.134)$ & $0.090)$ & $0.073)$ & $0.138)$ & $0.163)$ & $0.216)$ & $0.121)$ & $0.125)$ & $0.115)$ & $0.111)$ & $0.097)$ \\
& $(0.002$, & $(0.002$, & $(0.004$, & $(0.000$, & $(0.004$, & $(0.073$, & $(0.081$, & $(0.008$, & $(0.006$, & $(0.002$, & $(0.005$, & $(0.002$, \\
G1 & 0.049, & 0.017, & 0.010, & 0.001, & 0.047, & 0.135, & 0.153, & 0.034, & 0.025, & 0.035, & 0.053, & 0.029, \\
& $0.192)$ & $0.145)$ & $0.123)$ & $0.102)$ & $0.177)$ & $0.295)$ & $0.344)$ & $0.209)$ & $0.145)$ & $0.159)$ & $0.186)$ & $0.136)$ \\
& $(0.039$, & $(0.038$, & $(0.004$, & $(0.000$, & $(0.004$, & $(0.123$, & $(0.097$, & $(0.037$, & $(0.076$, & $(0.005$, & $(0.029$, & $(0.032$, \\
$G 2$ & 0.108, & 0.095, & 0.014, & 0.036, & 0.035, & 0.201, & 0.183, & 0.101, & 0.138, & 0.029, & 0.076, & 0.081, \\
& $0.276)$ & $0.243)$ & $0.139)$ & $0.156)$ & $0.191)$ & $0.356)$ & $0.368)$ & $0.291)$ & $0.312)$ & $0.156)$ & $0.219)$ & $0.218)$ \\
& $(0.041$, & $(0.023$, & $(0.006$, & $(0.000$, & $(0.006$, & $(0.078$, & $(0.122$, & $(0.038$, & $(0.045$, & $(0.005$, & $(0.007$, & $(0.005$, \\
$G 3$ & 0.101, & 0.057, & 0.013, & 0.001, & 0.029, & 0.132, & 0.198, & 0.090, & 0.101, & 0.022, & 0.025, & 0.020, \\
& $0.244)$ & $0.169)$ & $0.125)$ & $0.106)$ & $0.146)$ & $0.260)$ & $0.349)$ & $0.251)$ & $0.248)$ & $0.139)$ & $0.124)$ & $0.141)$ \\
& $(0.001$, & $(0.001$, & $(0.002$, & $(0.000$, & $(0.002$, & $(0.059$, & $(0.038$, & $(0.005$, & $(0.003$, & $(0.001$, & $(0.002$, & $(0.001$, \\
$G 4$ & 0.036, & 0.010, & 0.005, & 0.000, & 0.012, & 0.096, & 0.074, & 0.053, & 0.010, & 0.007, & 0.008, & 0.006, \\
& $0.149)$ & $0.112)$ & $0.098)$ & $0.081)$ & $0.116)$ & $0.225)$ & $0.218)$ & $0.197)$ & $0.136)$ & $0.111)$ & $0.115)$ & $0.076)$ \\
\hline
\end{tabular}

TABLE 12: Quantitated linguistic scoring matrix of sustainability criteria by considering probability for proposed projects.

\begin{tabular}{|c|c|c|c|c|c|c|c|c|c|c|c|c|}
\hline W & $\begin{array}{c}0.062 \\
E 1\end{array}$ & $\begin{array}{c}0.087 \\
E 2\end{array}$ & $\begin{array}{c}0.106 \\
E 3\end{array}$ & $\begin{array}{c}0.09 \\
E 4\end{array}$ & $\begin{array}{c}0.067 \\
S 1\end{array}$ & $\begin{array}{c}0.109 \\
S 2\end{array}$ & $\begin{array}{c}0.072 \\
S 3\end{array}$ & $\begin{array}{c}0.045 \\
S 4\end{array}$ & $\begin{array}{c}0.103 \\
G 1\end{array}$ & $\begin{array}{c}0.052 \\
G 2\end{array}$ & $\begin{array}{c}0.099 \\
G 3\end{array}$ & $\begin{array}{c}0.109 \\
G 4\end{array}$ \\
\hline$P 1$ & $\begin{array}{c}(1.235,1.3, \\
1.365)\end{array}$ & $\begin{array}{c}(19.627, \\
20.66 \\
21.693)\end{array}$ & $\begin{array}{c}(26 \\
29,33)\end{array}$ & $\begin{array}{l}0.45, \\
0.67 \\
0.89)\end{array}$ & $\begin{array}{l}(0,0 \\
0.11)\end{array}$ & $\begin{array}{l}0.22, \\
0.45, \\
0.67)\end{array}$ & $\begin{array}{c}(0.75,1 \\
1)\end{array}$ & $\begin{array}{l}(0,0 \\
0.22)\end{array}$ & $\begin{array}{l}(0,0, \\
0.22)\end{array}$ & $\begin{array}{l}0.22 \\
0.34 \\
0.45)\end{array}$ & $\begin{array}{l}0.22, \\
0.34 \\
0.45)\end{array}$ & $\begin{array}{l}0.67, \\
0.89, \\
0.89)\end{array}$ \\
\hline$P 2$ & $\begin{array}{c}(1.615,1.7 \\
1.785)\end{array}$ & $\begin{array}{c}(23.902, \\
25.16 \\
26.418)\end{array}$ & $\begin{array}{c}(32, \\
35,39)\end{array}$ & $\begin{array}{c}(0.47 \\
0.71 \\
0.95)\end{array}$ & $\begin{array}{c}(0,0.22 \\
0.45)\end{array}$ & $\begin{array}{l}(0,0 \\
0.18)\end{array}$ & $\begin{array}{l}(0.39 \\
0.58 \\
0.77)\end{array}$ & $\begin{array}{c}(0.25, \\
0.5,0.75)\end{array}$ & $\begin{array}{l}(0.47, \\
0.63, \\
0.63)\end{array}$ & $\begin{array}{l}(0.58 \\
0.77 \\
0.77)\end{array}$ & $\begin{array}{l}(0.47 \\
0.71 \\
0.95)\end{array}$ & $\begin{array}{l}(0,0 \\
0.22)\end{array}$ \\
\hline$P 3$ & $\begin{array}{c}(0.827, \\
0.87 \\
0.914)\end{array}$ & $\begin{array}{c}(14.44,15.2 \\
15.96)\end{array}$ & $\begin{array}{c}(14, \\
17,21)\end{array}$ & $\begin{array}{l}(0,0, \\
0.22)\end{array}$ & $\begin{array}{l}(0,0 \\
0.11)\end{array}$ & $\begin{array}{c}(0,0.16 \\
0.32)\end{array}$ & $\begin{array}{l}(0.71 \\
0.95 \\
0.95)\end{array}$ & $\begin{array}{l}(0.53 \\
0.71 \\
0.71)\end{array}$ & $\begin{array}{c}(0.75,1 \\
1)\end{array}$ & $\begin{array}{l}(0.22 \\
0.45 \\
0.67)\end{array}$ & $\begin{array}{l}(0,0 \\
0.24)\end{array}$ & $\begin{array}{l}(0.24, \\
0.47, \\
0.71)\end{array}$ \\
\hline$P 4$ & $\begin{array}{c}(1.881, \\
1.98, \\
2.079)\end{array}$ & $\begin{array}{c}(25.365 \\
26.7,28.035)\end{array}$ & $\begin{array}{c}(43, \\
46,50)\end{array}$ & $\begin{array}{l}(0.47, \\
0.71 \\
0.95)\end{array}$ & $\begin{array}{c}(0,0.25 \\
0.5)\end{array}$ & $\begin{array}{l}(0,0 \\
0.22)\end{array}$ & $\begin{array}{l}(0.34 \\
0.45 \\
0.45)\end{array}$ & $\begin{array}{c}(0,0.16 \\
0.32)\end{array}$ & $\begin{array}{l}(0.11 \\
0.22 \\
0.34)\end{array}$ & $\begin{array}{c}(0,0.16 \\
0.32)\end{array}$ & $\begin{array}{l}(0.58 \\
0.77 \\
0.77)\end{array}$ & $\begin{array}{l}(0.45, \\
0.67, \\
0.89)\end{array}$ \\
\hline P5 & $\begin{array}{c}(1.064, \\
1.12, \\
1.176)\end{array}$ & $\begin{array}{c}(18.459 \\
19.43 \\
20.402)\end{array}$ & $\begin{array}{c}(21, \\
24,28)\end{array}$ & $\begin{array}{l}(0,0, \\
0.18)\end{array}$ & $\begin{array}{l}(0,0 \\
0.19)\end{array}$ & $\begin{array}{c}(0,0.18, \\
0.35)\end{array}$ & $\begin{array}{c}(0,0.24 \\
0.47)\end{array}$ & $\begin{array}{l}(0,0 \\
0.24)\end{array}$ & $\begin{array}{l}(0,0, \\
0.24)\end{array}$ & $\begin{array}{c}(0.25 \\
0.5,0.75)\end{array}$ & $\begin{array}{l}(0,0 \\
0.22)\end{array}$ & $\begin{array}{l}(0,0, \\
0.25)\end{array}$ \\
\hline$P 6$ & $\begin{array}{c}(2.014, \\
2.12, \\
2.226)\end{array}$ & $\begin{array}{c}(28.595 \\
30.1,31.605)\end{array}$ & $\begin{array}{c}(48, \\
51,55)\end{array}$ & $\begin{array}{l}(0.47, \\
0.71 \\
0.95)\end{array}$ & $\begin{array}{l}(0.24, \\
0.47 \\
0.71)\end{array}$ & $\begin{array}{l}(0.24, \\
0.47 \\
0.71)\end{array}$ & $\begin{array}{l}(0.24 \\
0.47 \\
0.71)\end{array}$ & $\begin{array}{l}(0.11 \\
0.22 \\
0.34)\end{array}$ & $\begin{array}{l}(0.39 \\
0.58 \\
0.77)\end{array}$ & $\begin{array}{l}(0,0 \\
0.11)\end{array}$ & $\begin{array}{c}(0,0.19 \\
0.39)\end{array}$ & $\begin{array}{l}(0.18, \\
0.35, \\
0.53)\end{array}$ \\
\hline$P 7$ & $\begin{array}{c}(1.777, \\
1.87, \\
1.964)\end{array}$ & $\begin{array}{c}(22.582 \\
23.77 \\
24.959)\end{array}$ & $\begin{array}{c}(39, \\
42,46)\end{array}$ & $\begin{array}{l}(0,0, \\
0.22)\end{array}$ & $\begin{array}{c}(0,0.19 \\
0.39)\end{array}$ & $\begin{array}{l}(0,0 \\
0.16)\end{array}$ & $\begin{array}{c}(0,0.22, \\
0.45)\end{array}$ & $\begin{array}{l}(0.71 \\
0.95 \\
0.95)\end{array}$ & $\begin{array}{l}(0,0 \\
0.18)\end{array}$ & $\begin{array}{l}(0.18 \\
0.35 \\
0.53)\end{array}$ & $\begin{array}{l}(0.22 \\
0.34 \\
0.45)\end{array}$ & $\begin{array}{l}(0.45, \\
0.67, \\
0.89)\end{array}$ \\
\hline$P 8$ & $\begin{array}{c}(0.931, \\
0.98 \\
1.029)\end{array}$ & $\begin{array}{c}(16.084 \\
16.93 \\
17.777)\end{array}$ & $\begin{array}{c}(16 \\
19,23)\end{array}$ & $\begin{array}{l}(0.39 \\
0.58 \\
0.77)\end{array}$ & $\begin{array}{c}(0.75,1 \\
1)\end{array}$ & $\begin{array}{c}(0.34, \\
0.45 \\
0.45)\end{array}$ & $\begin{array}{l}(0.47 \\
0.63 \\
0.63)\end{array}$ & $\begin{array}{c}(0.5, \\
0.75,1)\end{array}$ & $\begin{array}{c}(0,0.19 \\
0.39)\end{array}$ & $\begin{array}{l}(0.47 \\
0.71 \\
0.95)\end{array}$ & $\begin{array}{l}(0,0 \\
0.24)\end{array}$ & $\begin{array}{l}(0.47, \\
0.71 \\
0.95)\end{array}$ \\
\hline
\end{tabular}

DEMATEL so as to specify key performance indicators (KPIs) for hospital management. Through their research, complex uncertain interrelation evaluations are determined by experts using Z-numbers, after which a DEMATEL approach is utilized to determine KPIs. They have applied their strategy to the case study of a rehabilitation hospital for which KPIs have been determined [60]. In another study, Ikram et al. have assessed a number of management and standardization criteria which have been prioritized using the AHP method, followed by prioritizing the alternatives via a fuzzy VIKOR approach. The outcomes of their study indicate that systematic management and standardization are the most influential criteria among other criteria assessed by their approach, the consideration of which is claimed by the authors of this research to be a great help to improve sustainable business practices [26]. Given ambiguity and uncertainty in human decisions, $\mathrm{Ma}$ et al. have developed a complex Pythagorean fuzzy VIKOR (CPF-VIKOR) method, which is capable of solving multicriteria decisionmaking problems by allowing linguistic terms that represent the opinions of experts. Their method has been tested on two numerical examples, the results of which have been compared with those from the CPF-TOPSIS, only to show the superiority of their presented approach [20]. In 2021, Akram et al. presented a complex spherical fuzzy model which is capable of dealing with two-dimensional information that suffers from ambiguity. The model is solved via complex spherical fuzzy VIKOR (CSF-VIKOR), and their proposed 
TABLE 13: Normalized scoring matrix.

\begin{tabular}{|c|c|c|c|c|c|c|c|c|c|c|c|c|}
\hline & $E 1$ & E2 & E3 & $E 4$ & $S 1$ & $S 2$ & S3 & $S 4$ & $G 1$ & $G 2$ & G3 & G4 \\
\hline$P 1$ & $\begin{array}{l}(0.464, \\
0.586, \\
0.708)\end{array}$ & $\begin{array}{c}(0.402, \\
0.55, \\
0.698)\end{array}$ & $\begin{array}{l}(0.122, \\
0.293 \\
0.463)\end{array}$ & $\begin{array}{c}(-0.442 \\
0.042 \\
0.526)\end{array}$ & $\begin{array}{c}(0.64,1 \\
1)\end{array}$ & $\begin{array}{c}(-0.465 \\
0.028 \\
0.69)\end{array}$ & $\begin{array}{l}(-0.25 \\
0,0.25)\end{array}$ & $\begin{array}{c}(0.49 \\
0.95,1)\end{array}$ & $\begin{array}{l}(-0.18 \\
0,0.22)\end{array}$ & $\begin{array}{l}(0.137, \\
0.453, \\
0.768)\end{array}$ & $\begin{array}{c}(0,0.358 \\
0.474)\end{array}$ & $\begin{array}{l}(-0.232 \\
0,0.295)\end{array}$ \\
\hline$P 2$ & $\begin{array}{c}(0.164, \\
0.3, \\
0.437)\end{array}$ & $\begin{array}{l}(0.127 \\
0.288 \\
0.449)\end{array}$ & $\begin{array}{c}(0.268 \\
0.439 \\
0.61)\end{array}$ & $\begin{array}{l}(-0.505 \\
0,0.505)\end{array}$ & $\begin{array}{c}(0.3, \\
0.78,1)\end{array}$ & $\begin{array}{c}(0.225, \\
0.662,1)\end{array}$ & $\begin{array}{c}(-0.02 \\
0.42 \\
0.61)\end{array}$ & $\begin{array}{c}(-0.04 \\
0.45 \\
0.75)\end{array}$ & $\begin{array}{l}(0.29 \\
0.63 \\
0.63)\end{array}$ & $\begin{array}{c}(-0.2,0 \\
0.389)\end{array}$ & $\begin{array}{c}(0.263, \\
0.747,1)\end{array}$ & $\begin{array}{c}(0.474, \\
0.937,1)\end{array}$ \\
\hline$P 3$ & $\begin{array}{c}(0.786 \\
0.893,1)\end{array}$ & $\begin{array}{c}(0.736, \\
0.868,1)\end{array}$ & $\begin{array}{l}(-0.171 \\
0,0.171)\end{array}$ & $\begin{array}{c}(0.263, \\
0.747,1)\end{array}$ & $\begin{array}{c}(0.64,1 \\
1)\end{array}$ & $\begin{array}{c}(0.028, \\
0.437,1)\end{array}$ & $\begin{array}{l}(-0.2 \\
0.05 \\
0.29)\end{array}$ & $\begin{array}{c}(0,0.24 \\
0.47)\end{array}$ & $\begin{array}{c}(0.57,1 \\
1)\end{array}$ & $\begin{array}{c}(-0.095 \\
0.337 \\
0.768)\end{array}$ & $\begin{array}{l}(-0.232 \\
0,0.253)\end{array}$ & $\begin{array}{c}(-0.042 \\
0.442 \\
0.747)\end{array}$ \\
\hline P4 & $\begin{array}{c}(-0.046 \\
0.1 \\
0.247)\end{array}$ & $\begin{array}{l}(0.033, \\
0.198, \\
0.364)\end{array}$ & $\begin{array}{l}(0.537, \\
0.707, \\
0.878)\end{array}$ & $\begin{array}{l}(-0.505 \\
0,0.505)\end{array}$ & $\begin{array}{c}(0.25 \\
0.75,1)\end{array}$ & $\begin{array}{c}(0.169, \\
0.662,1)\end{array}$ & $\begin{array}{l}(0.3 \\
0.55 \\
0.66)\end{array}$ & $\begin{array}{c}(0.39, \\
0.79,1)\end{array}$ & $\begin{array}{c}(-0.07 \\
0.22 \\
0.34)\end{array}$ & $\begin{array}{c}(0.274, \\
0.642,1)\end{array}$ & $\begin{array}{l}(0.379, \\
0.811, \\
0.811)\end{array}$ & $\begin{array}{c}(-0.232 \\
0.232 \\
0.526)\end{array}$ \\
\hline P5 & $\begin{array}{c}(0.599 \\
0.715 \\
0.831)\end{array}$ & $\begin{array}{l}(0.477, \\
0.622, \\
0.766)\end{array}$ & $\begin{array}{c}(0,0.171 \\
0.341)\end{array}$ & $\begin{array}{c}(0.305, \\
0.747,1)\end{array}$ & $\begin{array}{c}(0.56,1 \\
1)\end{array}$ & $\begin{array}{c}(-0.014 \\
0.408,1)\end{array}$ & $\begin{array}{c}(0.28, \\
0.76,1)\end{array}$ & $\begin{array}{c}(0.47 \\
0.95,1)\end{array}$ & $\begin{array}{c}(-0.18 \\
0,0.24)\end{array}$ & $\begin{array}{c}(-0.179 \\
0.284 \\
0.737)\end{array}$ & $\begin{array}{l}(-0.232 \\
0,0.232)\end{array}$ & $\begin{array}{c}(0.442, \\
0.937,1)\end{array}$ \\
\hline P6 & $\begin{array}{l}(-0.152 \\
0,0.152)\end{array}$ & $\begin{array}{l}(-0.175 \\
0,0.175)\end{array}$ & $\begin{array}{c}(0.659, \\
0.829,1)\end{array}$ & $\begin{array}{l}(-0.505 \\
0,0.505)\end{array}$ & $\begin{array}{l}(0.04 \\
0.53 \\
0.76)\end{array}$ & $\begin{array}{c}(-0.521 \\
0,0.662)\end{array}$ & $\begin{array}{l}(0.04 \\
0.53 \\
0.76)\end{array}$ & $\begin{array}{l}(0.37 \\
0.73 \\
0.89)\end{array}$ & $\begin{array}{l}(0.21 \\
0.58 \\
0.77)\end{array}$ & $\begin{array}{c}(0.495, \\
0.811,1)\end{array}$ & $\begin{array}{c}(-0.232 \\
0.2 \\
0.411)\end{array}$ & $\begin{array}{c}(0.147 \\
0.568 \\
0.811)\end{array}$ \\
\hline P7 & $\begin{array}{l}(0.036 \\
0.179 \\
0.321)\end{array}$ & $\begin{array}{l}(0.212, \\
0.369, \\
0.526)\end{array}$ & $\begin{array}{c}(0.439 \\
0.61 \\
0.78)\end{array}$ & $\begin{array}{c}(0.263, \\
0.747,1)\end{array}$ & $\begin{array}{c}(0.36 \\
0.81,1)\end{array}$ & $\begin{array}{c}(0.254, \\
0.662,1)\end{array}$ & $\begin{array}{c}(0.3, \\
0.78,1)\end{array}$ & $\begin{array}{l}(-0.24 \\
0,0.29)\end{array}$ & $\begin{array}{l}(-0.18 \\
0,0.18)\end{array}$ & $\begin{array}{c}(0.053 \\
0.442 \\
0.811)\end{array}$ & $\begin{array}{c}(0,0.358 \\
0.474)\end{array}$ & $\begin{array}{c}(-0.232 \\
0.232 \\
0.526)\end{array}$ \\
\hline$P 8$ & $\begin{array}{l}(0.704, \\
0.815 \\
0.926) \\
\end{array}$ & $\begin{array}{l}(0.63, \\
0.767, \\
0.904)\end{array}$ & $\begin{array}{c}(-0.122 \\
0.049 \\
0.22)\end{array}$ & $\begin{array}{c}(-0.316 \\
0.137 \\
0.589)\end{array}$ & $\begin{array}{l}(-0.25 \\
0,0.25)\end{array}$ & $\begin{array}{c}(-0.155 \\
0.028 \\
0.521)\end{array}$ & $\begin{array}{l}(0.12, \\
0.37 \\
0.53)\end{array}$ & $\begin{array}{c}(-0.29 \\
0.2,0.5)\end{array}$ & $\begin{array}{c}(-0.18 \\
0.19 \\
0.39)\end{array}$ & $\begin{array}{c}(-0.389 \\
0.063 \\
0.505)\end{array}$ & $\begin{array}{l}(-0.232 \\
0,0.253)\end{array}$ & $\begin{array}{c}(-0.295 \\
0.189 \\
0.505)\end{array}$ \\
\hline
\end{tabular}

strategy has been tested on a numerical study associated with business, which ranks the objectives of an advertisement on Facebook. The results have been compared with those from the spherical fuzzy VIKOR (SF-VIKOR) method, leading to the fact that the CSF-VIKOR has been superior in performance [61].

2.5. Fuzzy and Z-Number Information. First, fuzzy sets were introduced in $[29,62]$. Fuzzy sets introduce the concept of membership functionality, which deals with different linguistic variables and has many applications such as the field of medicine [63], measuring similarity for uncertain information [64], multidisciplinary group decision-making [65], selection of backup options [64], and review of supply chains under uncertainty data [66]. The fuzzy sets are summarized as follows.

Definition 1. Fuzzy sets $A$ are defined on a primary set $X$ and are shown in the following equation [29]:

$$
A=\left\{\left\langle x, \mu_{A}(x)\right\rangle \mid x \in X\right\}
$$

where $\mu_{A}: X \longrightarrow[0,1]$ is the membership function of $A$. The value of $\mu_{A}(x)$ describes the degree of $x \in X$ affiliation in $A$. Sometimes, a special fuzzy set, for example, a triangular fuzzy number and a trapezoidal fuzzy number are used to express the main idea of the experts, we define them as follows.

$\widetilde{A}$ is called a triangular fuzzy number in $R$ whenever the membership function is defined as follows:

$$
\mu_{\tilde{A}}(x)= \begin{cases}\frac{x-a_{1}}{a_{2}-a_{1}}, & a_{1} \leq x \leq a_{2}, \\ 1, & x=a_{2}, \\ \frac{a_{3}-x}{a_{3}-a_{2}}, & a_{2} \leq x \leq a_{3}, \\ 0, & \text { o.w. }\end{cases}
$$

We display triangular fuzzy numbers as $\widetilde{A}=\left(a_{1}, a_{2}, a_{3}\right)$ and use $F(\mathbb{R})$ to represent the set of fuzzy triangular numbers. It should be noted that the upper bound represented by $a_{3}$ is the maximum value that $\widetilde{A}$ can take and the lower bound shown by $a_{1}$ is the minimum value that $\widetilde{A}$ takes, and $a_{2}$ is the most probable value of a fuzzy number. If we set $m=a_{2}$, then $m$ represents the value of the viewpoint or the midpoint, and $\alpha=a_{2}-a_{1}$ shows the left extension and $\beta=$ $a_{3}-a_{2}$ shows the right extension of the triangular fuzzy number $\widetilde{A}=\left(a_{1}, a_{2}, a_{3}\right)$. Therefore, the fuzzy triangular number $\widetilde{A}$ can also be represented by the triple $\widetilde{A}=(m, \alpha, \beta)$. The fuzzy triangular number $\widetilde{A}=\left(a_{1}, a_{2}, a_{3}\right)$ is displayed in geometric space as follows (Figure 1 ).

Given the triangular membership function, it is clear that if $x$ is between $a_{1}$ and $a_{2}$, then the larger it gets, the greater the degree of its membership, so that for $x=a_{2}$, the degree of membership is equal to one. If $x$ is between $a_{2}$ and $a_{3}$, then the larger it gets, the smaller the membership degree will be, and finally, when $x=a_{3}$, the membership degree will be zero. In fact, a fuzzy triangular number indicates the boundary among large, low, and medium values. 
TABLE 14: Quantified probabilistic linguistic scoring variable for sustainability criteria for proposed projects.

\begin{tabular}{|c|c|c|c|c|c|c|c|c|c|c|c|c|}
\hline & $E 1$ & $E 2$ & E3 & $E 4$ & $S 1$ & $S 2$ & S3 & $S 4$ & $G 1$ & G2 & G3 & G4 \\
\hline$P 1$ & $\begin{array}{l}(0.238, \\
0.475, \\
0.713)\end{array}$ & $\begin{array}{c}(0.475 \\
0.713 \\
0.95)\end{array}$ & $\begin{array}{l}(0.223, \\
0.445, \\
0.668)\end{array}$ & $\begin{array}{l}(0.223, \\
0.445, \\
0.668)\end{array}$ & $\begin{array}{c}(0.668, \\
0.89 \\
0.89)\end{array}$ & $\begin{array}{l}(0.223, \\
0.445, \\
0.668)\end{array}$ & $\begin{array}{c}(0.5 \\
0.75,1)\end{array}$ & $\begin{array}{c}(0.5 \\
0.75,1)\end{array}$ & $\begin{array}{c}(0.75,1 \\
1)\end{array}$ & $\begin{array}{c}(0.475 \\
0.713 \\
0.95)\end{array}$ & $\begin{array}{c}(0.75,1 \\
1)\end{array}$ & $\begin{array}{l}(0.238, \\
0.475, \\
0.713)\end{array}$ \\
\hline$P 2$ & $\begin{array}{c}(0,0.223 \\
0.445)\end{array}$ & $\begin{array}{c}(0.385 \\
0.578 \\
0.77)\end{array}$ & $\begin{array}{c}(0,0.223 \\
0.445)\end{array}$ & $\begin{array}{c}(0,0, \\
0.193)\end{array}$ & $\begin{array}{c}(0,0.193 \\
0.385)\end{array}$ & $\begin{array}{l}(0.238, \\
0.475, \\
0.713)\end{array}$ & $\begin{array}{c}(0.445, \\
0.668 \\
0.89)\end{array}$ & $\begin{array}{l}(0.178, \\
0.355, \\
0.533)\end{array}$ & $\begin{array}{c}(0,0, \\
0.223)\end{array}$ & $\begin{array}{c}(0.475 \\
0.713 \\
0.95)\end{array}$ & $\begin{array}{c}(0,0, \\
0.223)\end{array}$ & $\begin{array}{c}(0,0, \\
0.193)\end{array}$ \\
\hline$P 3$ & $\begin{array}{c}(0.578, \\
0.77 \\
0.77)\end{array}$ & $\begin{array}{c}(0,0.158 \\
0.315)\end{array}$ & $\begin{array}{c}(0.355 \\
0.533 \\
0.71)\end{array}$ & $\begin{array}{c}(0.578, \\
0.77 \\
0.77)\end{array}$ & $\begin{array}{c}(0.355 \\
0.533 \\
0.71)\end{array}$ & $\begin{array}{c}(0.385 \\
0.578, \\
0.77)\end{array}$ & $\begin{array}{c}(0,0.223 \\
0.445)\end{array}$ & $\begin{array}{c}(0,0.178 \\
0.355)\end{array}$ & $\begin{array}{c}(0,0.193 \\
0.385)\end{array}$ & $\begin{array}{c}(0.473, \\
0.63 \\
0.63)\end{array}$ & $\begin{array}{c}(0,0.193 \\
0.385)\end{array}$ & $\begin{array}{c}(0.445, \\
0.668, \\
0.89)\end{array}$ \\
\hline$P 4$ & $\begin{array}{c}(0.475, \\
0.713, \\
0.95)\end{array}$ & $\begin{array}{c}(0,0.223 \\
0.445)\end{array}$ & $\begin{array}{c}(0.445, \\
0.668 \\
0.89)\end{array}$ & $\begin{array}{l}(0.223, \\
0.445, \\
0.668)\end{array}$ & $\begin{array}{l}(0,0 \\
0.25)\end{array}$ & $\begin{array}{c}(0.5, \\
0.75,1)\end{array}$ & $\begin{array}{l}(0.238 \\
0.475 \\
0.713)\end{array}$ & $\begin{array}{c}(0.25 \\
0.5,0.75)\end{array}$ & $\begin{array}{c}(0,0.223 \\
0.445)\end{array}$ & $\begin{array}{c}(0.445, \\
0.668, \\
0.89)\end{array}$ & $\begin{array}{l}(0.238 \\
0.475 \\
0.713)\end{array}$ & $\begin{array}{c}(0.475, \\
0.713, \\
0.95)\end{array}$ \\
\hline P5 & $\begin{array}{l}(0.238, \\
0.475 \\
0.713)\end{array}$ & $\begin{array}{l}(0.158, \\
0.315, \\
0.473)\end{array}$ & $\begin{array}{c}(0,0 \\
0.158)\end{array}$ & $\begin{array}{l}(0.193, \\
0.385, \\
0.578)\end{array}$ & $\begin{array}{c}(0,0.158 \\
0.315)\end{array}$ & $\begin{array}{c}(0,0, \\
0.238)\end{array}$ & $\begin{array}{c}(0.355 \\
0.533 \\
0.71)\end{array}$ & $\begin{array}{c}(0.5, \\
0.75,1)\end{array}$ & $\begin{array}{c}(0,0.193 \\
0.385)\end{array}$ & $\begin{array}{l}(0.238, \\
0.475 \\
0.713)\end{array}$ & $\begin{array}{c}(0,0.193 \\
0.385)\end{array}$ & $\begin{array}{c}(0,0, \\
0.223)\end{array}$ \\
\hline$P 6$ & $\begin{array}{c}(0.533, \\
0.71 \\
0.71)\end{array}$ & $\begin{array}{c}(0.578, \\
0.77 \\
0.77)\end{array}$ & $\begin{array}{c}(0.445, \\
0.668, \\
0.89)\end{array}$ & $\begin{array}{c}(0,0, \\
0.238)\end{array}$ & $\begin{array}{l}(0.178, \\
0.355 \\
0.533)\end{array}$ & $\begin{array}{c}(0.385, \\
0.578, \\
0.77)\end{array}$ & $\begin{array}{c}(0,0.178, \\
0.355)\end{array}$ & $\begin{array}{c}(0.475 \\
0.713 \\
0.95)\end{array}$ & $\begin{array}{c}(0.355 \\
0.533 \\
0.71)\end{array}$ & $\begin{array}{c}(0,0.178 \\
0.355)\end{array}$ & $\begin{array}{c}(0.475 \\
0.713 \\
0.95)\end{array}$ & $\begin{array}{l}(0.193, \\
0.385, \\
0.578)\end{array}$ \\
\hline$P 7$ & $\begin{array}{c}(0,0 \\
0.193)\end{array}$ & $\begin{array}{l}(0.193, \\
0.385, \\
0.578)\end{array}$ & $\begin{array}{c}(0.5, \\
0.75,1)\end{array}$ & $\begin{array}{l}(0.238, \\
0.475, \\
0.713)\end{array}$ & $\begin{array}{l}(0.223, \\
0.445 \\
0.668)\end{array}$ & $\begin{array}{c}(0,0.193 \\
0.385)\end{array}$ & $\begin{array}{c}(0,0.193 \\
0.385)\end{array}$ & $\begin{array}{c}(0.5, \\
0.75,1)\end{array}$ & $\begin{array}{c}(0,0 \\
0.158)\end{array}$ & $\begin{array}{l}(0.158, \\
0.315, \\
0.473)\end{array}$ & $\begin{array}{c}(0,0.193 \\
0.385)\end{array}$ & $\begin{array}{c}(0,0.178 \\
0.355)\end{array}$ \\
\hline P8 & $\begin{array}{c}(0.445, \\
0.668, \\
0.89)\end{array}$ & $\begin{array}{c}(0.475, \\
0.713, \\
0.95)\end{array}$ & $\begin{array}{c}(0.75,1 \\
1)\end{array}$ & $\begin{array}{c}(0.668 \\
0.89 \\
0.89)\end{array}$ & $\begin{array}{c}(0.5 \\
0.75,1)\end{array}$ & $\begin{array}{c}(0.475, \\
0.713, \\
0.95)\end{array}$ & $\begin{array}{c}(0.475 \\
0.713 \\
0.95)\end{array}$ & $\begin{array}{c}(0.713 \\
0.95 \\
0.95)\end{array}$ & $\begin{array}{c}(0.475 \\
0.713 \\
0.95)\end{array}$ & $\begin{array}{c}(0.475 \\
0.713 \\
0.95)\end{array}$ & $\begin{array}{c}(0.475 \\
0.713 \\
0.95)\end{array}$ & $\begin{array}{c}(0.445, \\
0.668, \\
0.89)\end{array}$ \\
\hline$P 9$ & $\begin{array}{l}(0 . \\
0.3 \\
0.5\end{array}$ & $\begin{array}{l}(0.223, \\
0.445, \\
0.668)\end{array}$ & $\begin{array}{c}(0.385 \\
0.578 \\
0.77)\end{array}$ & $\begin{array}{c}(0.475, \\
0.713 \\
0.95)\end{array}$ & $\begin{array}{l}(0.223, \\
0.445, \\
0.668)\end{array}$ & $\begin{array}{c}(0.315, \\
0.473, \\
0.63)\end{array}$ & $\begin{array}{c}(0.385 \\
0.578 \\
0.77)\end{array}$ & $\begin{array}{c}(0.315, \\
0.473, \\
0.63)\end{array}$ & $\begin{array}{c}(0.475 \\
0.713 \\
0.95)\end{array}$ & $\begin{array}{c}(0.668 \\
0.89 \\
0.89)\end{array}$ & $\begin{array}{c}(0.473 \\
0.63 \\
0.63)\end{array}$ & $\begin{array}{c}(0.315, \\
0.473, \\
0.63)\end{array}$ \\
\hline$P 10$ & $\begin{array}{l}(0.2 \\
0.4 \\
0.7\end{array}$ & $\begin{array}{l}(0,0, \\
0.25)\end{array}$ & $\begin{array}{c}(0.25 \\
0.5,0.75)\end{array}$ & $\begin{array}{l}(0.223, \\
0.445, \\
0.668)\end{array}$ & $\begin{array}{c}(0,0.223 \\
0.445)\end{array}$ & $\begin{array}{l}(0.223, \\
0.445, \\
0.668)\end{array}$ & $\begin{array}{c}(0.25 \\
0.5,0.75)\end{array}$ & $\begin{array}{c}(0,0.238 \\
0.475)\end{array}$ & $\begin{array}{c}(0.25 \\
0.5,0.75)\end{array}$ & $\begin{array}{c}(0,0.223 \\
0.445)\end{array}$ & $\begin{array}{c}(0,0, \\
0.238)\end{array}$ & $\begin{array}{c}(0,0, \\
0.238)\end{array}$ \\
\hline$P 11$ & $\begin{array}{c}(0.713 \\
0.95 \\
0.95)\end{array}$ & $\begin{array}{c}(0,0.25 \\
0.5)\end{array}$ & $\begin{array}{c}(0.445, \\
0.668, \\
0.89)\end{array}$ & $\begin{array}{c}(0,0.238 \\
0.475)\end{array}$ & $\begin{array}{c}(0.533 \\
0.71 \\
0.71)\end{array}$ & $\begin{array}{c}(0,0.178 \\
0.355)\end{array}$ & $\begin{array}{l}(0.238 \\
0.475 \\
0.713)\end{array}$ & $\begin{array}{c}(0,0.223 \\
0.445)\end{array}$ & $\begin{array}{c}(0.385 \\
0.578 \\
0.77)\end{array}$ & $\begin{array}{c}(0.385 \\
0.578 \\
0.77)\end{array}$ & & $\begin{array}{c}(0,0.25 \\
0.5)\end{array}$ \\
\hline$P 12$ & $\begin{array}{l}(0.178, \\
0.355, \\
0.533)\end{array}$ & $\begin{array}{c}(0,0.223 \\
0.445)\end{array}$ & $\begin{array}{l}(0.223, \\
0.445, \\
0.668)\end{array}$ & $\begin{array}{l}(0.223, \\
0.445, \\
0.668)\end{array}$ & $\begin{array}{c}(0.385 \\
0.578 \\
0.77)\end{array}$ & $\begin{array}{c}(0,0.223 \\
0.445)\end{array}$ & $\begin{array}{c}(0.445, \\
0.668 \\
0.89)\end{array}$ & $\begin{array}{l}(0.223, \\
0.445, \\
0.668)\end{array}$ & $\begin{array}{c}(0,0.223 \\
0.445)\end{array}$ & $\begin{array}{c}(0,0.223 \\
0.445)\end{array}$ & $\begin{array}{c}(0.445, \\
0.668 \\
0.89)\end{array}$ & $\begin{array}{l}(0.193, \\
0.385, \\
0.578)\end{array}$ \\
\hline$P 13$ & $\begin{array}{c}(0,0, \\
0.223)\end{array}$ & $\begin{array}{c}(0,0, \\
0.178)\end{array}$ & $\begin{array}{c}(0.385 \\
0.578 \\
0.77)\end{array}$ & $\begin{array}{c}(0,0.238 \\
0.475)\end{array}$ & $\begin{array}{c}(0.445 \\
0.668 \\
0.89)\end{array}$ & $\begin{array}{c}(0,0, \\
0.193)\end{array}$ & $\begin{array}{c}(0,0.238 \\
0.475)\end{array}$ & $\begin{array}{l}(0.223, \\
0.445, \\
0.668)\end{array}$ & $\begin{array}{c}(0,0, \\
0.223)\end{array}$ & $\begin{array}{c}(0.385 \\
0.578 \\
0.77)\end{array}$ & $\begin{array}{c}(0,0.193 \\
0.385)\end{array}$ & $\begin{array}{c}(0.355 \\
0.533 \\
0.71)\end{array}$ \\
\hline P14 & & $\begin{array}{l}(0.178, \\
0.355, \\
0.533)\end{array}$ & $\begin{array}{c}(0.5, \\
0.75,1)\end{array}$ & $\begin{array}{l}(0.223, \\
0.445, \\
0.668)\end{array}$ & $\begin{array}{c}(0.445, \\
0.668 \\
0.89)\end{array}$ & $\begin{array}{c}(0,0.238 \\
0.475)\end{array}$ & $\begin{array}{l}(0,0 \\
0.25)\end{array}$ & $\begin{array}{c}(0,0.25 \\
0.5)\end{array}$ & $\begin{array}{c}(0,0 \\
0.178)\end{array}$ & $\begin{array}{l}(0.223, \\
0.445, \\
0.668)\end{array}$ & $\begin{array}{c}(0.385 \\
0.578 \\
0.77)\end{array}$ & $\begin{array}{l}(0.193, \\
0.385, \\
0.578)\end{array}$ \\
\hline$P 15$ & $\begin{array}{l}(0.238 \\
0.475 \\
0.713)\end{array}$ & $\begin{array}{l}(0,0 \\
0.25)\end{array}$ & $\begin{array}{c}(0.25 \\
0.5,0.75)\end{array}$ & $\begin{array}{c}(0,0, \\
0.223)\end{array}$ & $\begin{array}{c}(0,0.238 \\
0.475)\end{array}$ & $\begin{array}{c}(0.5, \\
0.75,1)\end{array}$ & $\begin{array}{c}(0,0.223 \\
0.445)\end{array}$ & $\begin{array}{c}(0.713, \\
0.95 \\
0.95)\end{array}$ & $\begin{array}{c}(0.445, \\
0.668, \\
0.89)\end{array}$ & $\begin{array}{c}(0,0.223 \\
0.445)\end{array}$ & $\begin{array}{c}(0,0.223 \\
0.445)\end{array}$ & $\begin{array}{c}(0.668 \\
0.89 \\
0.89)\end{array}$ \\
\hline$P 16$ & $\begin{array}{c}(0.445, \\
0.668, \\
0.89)\end{array}$ & $\begin{array}{l}(0.238, \\
0.475, \\
0.713)\end{array}$ & $\begin{array}{c}(0.475 \\
0.713 \\
0.95)\end{array}$ & $\begin{array}{l}(0.193, \\
0.385, \\
0.578)\end{array}$ & $\begin{array}{c}(0.315, \\
0.473 \\
0.63)\end{array}$ & $\begin{array}{l}(0.178, \\
0.355, \\
0.533)\end{array}$ & $\begin{array}{c}(0.578, \\
0.77 \\
0.77)\end{array}$ & $\begin{array}{l}(0.238, \\
0.475, \\
0.713)\end{array}$ & $\begin{array}{c}(0.25 \\
0.5,0.75)\end{array}$ & $\begin{array}{c}(0.533 \\
0.71 \\
0.71)\end{array}$ & $\begin{array}{l}(0.193, \\
0.385, \\
0.578)\end{array}$ & $\begin{array}{l}(0.178, \\
0.355, \\
0.533)\end{array}$ \\
\hline$P 17$ & $\begin{array}{c}(0,0.193 \\
0.385)\end{array}$ & $\begin{array}{c}(0.385, \\
0.578, \\
0.77)\end{array}$ & $\begin{array}{c}(0,0.178 \\
0.355)\end{array}$ & $\begin{array}{l}(0.178, \\
0.355, \\
0.533)\end{array}$ & $\begin{array}{c}(0.475 \\
0.713 \\
0.95)\end{array}$ & $\begin{array}{l}(0.238, \\
0.475, \\
0.713)\end{array}$ & $\begin{array}{l}(0.193, \\
0.385 \\
0.578)\end{array}$ & $\begin{array}{c}(0,0.238 \\
0.475)\end{array}$ & $\begin{array}{c}(0.475 \\
0.713 \\
0.95)\end{array}$ & $\begin{array}{c}(0,0.193 \\
0.385)\end{array}$ & $\begin{array}{l}(0.178, \\
0.355, \\
0.533)\end{array}$ & $\begin{array}{c}(0,0.178 \\
0.355)\end{array}$ \\
\hline$P 18$ & $\begin{array}{c}(0.25 \\
0.5,0.75)\end{array}$ & $\begin{array}{c}(0.475 \\
0.713, \\
0.95)\end{array}$ & $\begin{array}{c}(0.713 \\
0.95 \\
0.95)\end{array}$ & $\begin{array}{c}(0.355, \\
0.533, \\
0.71)\end{array}$ & $\begin{array}{c}(0.315 \\
0.473 \\
0.63)\end{array}$ & $\begin{array}{c}(0.25 \\
0.5,0.75)\end{array}$ & $\begin{array}{c}(0,0.25 \\
0.5)\end{array}$ & $\begin{array}{l}(0.178, \\
0.355, \\
0.533)\end{array}$ & $\begin{array}{c}(0,0.193 \\
0.385)\end{array}$ & $\begin{array}{c}(0.445, \\
0.668, \\
0.89)\end{array}$ & $\begin{array}{l}(0.158, \\
0.315, \\
0.473)\end{array}$ & $\begin{array}{c}(0.355 \\
0.533 \\
0.71)\end{array}$ \\
\hline P19 & $\begin{array}{l}(0.193, \\
0.385, \\
0.578)\end{array}$ & $\begin{array}{c}(0,0, \\
0.178)\end{array}$ & $\begin{array}{c}(0,0, \\
0.178)\end{array}$ & $\begin{array}{c}(0.475, \\
0.713 \\
0.95)\end{array}$ & $\begin{array}{c}(0,0.25 \\
0.5)\end{array}$ & $\begin{array}{l}(0.178, \\
0.355, \\
0.533)\end{array}$ & $\begin{array}{c}(0.385, \\
0.578 \\
0.77)\end{array}$ & $\begin{array}{c}(0,0.193 \\
0.385)\end{array}$ & $\begin{array}{c}(0.475 \\
0.713, \\
0.95)\end{array}$ & $\begin{array}{c}(0,0, \\
0.223)\end{array}$ & $\begin{array}{l}(0.238, \\
0.475, \\
0.713)\end{array}$ & $\begin{array}{c}(0,0.238 \\
0.475)\end{array}$ \\
\hline$P 20$ & $\begin{array}{c}(0.75,1 \\
1)\end{array}$ & $\begin{array}{l}(0,0 \\
0.25)\end{array}$ & $\begin{array}{c}(0.445, \\
0.668, \\
0.89)\end{array}$ & $\begin{array}{l}(0.223, \\
0.445, \\
0.668)\end{array}$ & $\begin{array}{c}(0.445, \\
0.668, \\
0.89)\end{array}$ & $\begin{array}{c}(0.75,1 \\
1)\end{array}$ & $\begin{array}{l}(0.223 \\
0.445 \\
0.668)\end{array}$ & $\begin{array}{c}(0.475 \\
0.713 \\
0.95)\end{array}$ & $\begin{array}{c}(0,0, \\
0.238)\end{array}$ & $\begin{array}{c}(0,0.238, \\
0.475)\end{array}$ & $\begin{array}{l}(0.223, \\
0.445, \\
0.668)\end{array}$ & $\begin{array}{c}(0.25 \\
0.5,0.75)\end{array}$ \\
\hline
\end{tabular}


TABLE 15: Comparison matrix of linguistic variable in Z-number condition.

\begin{tabular}{lccc}
\hline & Economic & Social & Environment \\
\hline Economic & & H-W & VH-L \\
Social & VL-W & & H-W \\
Environment & H-FI & N-ML & \\
\hline
\end{tabular}

TABLE 16: Comparison matrix of the linguistic variable of sustainability dimensions in Z-number condition.

\begin{tabular}{lccr}
\hline & Economic & Social & \multicolumn{1}{c}{ Environment } \\
\hline Economic & $(0.000,0.000,0.000)$ & $(0.350,0.530,0.710)$ & $(0.670,0.890,0.890)$ \\
Social & $(0.000,0.190,0.390)$ & $(0.000,0.000,0.000)$ & $(0.350,0.530,0.710)$ \\
Environment & $(0.320,0.470,0.630)$ & $(0.000,0.000,0.240)$ & $(0.000,0.000,0.000)$ \\
\hline
\end{tabular}

TABLE 17: Normalized matrix of sustainability dimensions in Z-number condition.

\begin{tabular}{lcrr}
\hline & Economic & Social & \multicolumn{1}{c}{ Environment } \\
\hline Economic & $(0.000,0.000,0.000)$ & $(0.219,0.331,0.444)$ & $(0.419,0.556,0.556)$ \\
Social & $(0.000,0.119,0.244)$ & $(0.000,0.000,0.000)$ & $(0.219,0.331,0.444)$ \\
Environment & $(0.200,0.294,0.394)$ & $(0.000,0.000,0.150)$ & $(0.000,0.000,0.000)$ \\
\hline
\end{tabular}

TABLE 18: Total relationship matrix of sustainability dimensions in Z-number condition.

\begin{tabular}{lcrr}
\hline & Economic & Social & \multicolumn{1}{c}{ Environment } \\
\hline Economic & $(0.103,0.307,0.836)$ & $(0.241,0.433,1.037)$ & $(0.515,0.871,1.482)$ \\
Social & $(0.048,0.282,0.823)$ & $(0.011,0.094,0.536)$ & $(0.241,0.519,1.140)$ \\
Environment & $(0.221,0.384,0.846)$ & $(0.048,0.127,0.639)$ & $(0.103,0.256,0.754)$ \\
\hline
\end{tabular}

TABLE 19: Importance weight of sustainability dimensions in Z-number condition.

\begin{tabular}{|c|c|c|c|c|c|c|}
\hline Dimension & $\widetilde{D}_{\mathbf{i}}+\widetilde{R}_{\mathbf{i}}$ & $\left(\widetilde{D}_{\mathbf{i}}+\widetilde{R}_{\mathbf{i}}\right)^{\text {def }}$ & $\widetilde{D}_{\mathbf{i}}-\widetilde{R}_{\mathbf{i}}$ & $\left(\widetilde{D}_{\mathbf{i}}-\widetilde{R}_{\mathbf{i}}\right)^{\text {def }}$ & Influence & $\mathrm{W}_{\mathrm{i}}^{\text {goal }}$ \\
\hline Economic & $(1.231,2.584,5.860)$ & 3.065 & $(-1.647,0.637,2.983)$ & 0.653 & Influence & 0.378 \\
\hline Social & $(0.600,1.549,4.711)$ & 2.102 & $(-1.912,0.242,2.199)$ & 0.193 & Influence & 0.255 \\
\hline Environment & $(1.231,2.412,5.615)$ & 2.918 & $(-3.004,-0.879,1.381)$ & -0.845 & Be-influence & 0.367 \\
\hline
\end{tabular}

Definition 2. mathematical operations on triangular fuzzy numbers). For fuzzy triangular numbers, $\widetilde{A}=\left(a_{1}, a_{2}\right.$, $\left.a_{3}\right)=\left(m_{1}, \alpha_{1}, \beta_{1}\right)$ and $\widetilde{B}=\left(b_{1}, b_{2}, b_{3}\right)=\left(m_{2}, \alpha_{2}, \beta_{2}\right)$, the mathematical operations on these numbers are defined as follows:

$$
\begin{aligned}
(1) \widetilde{A}+\widetilde{B} & =\left(a_{1}, a_{2}, a_{3}\right)+\left(b_{1}, b_{2}, b_{3}\right) \\
& =\left(m_{1}, \alpha_{1}, \beta_{1}\right)+\left(m_{2}, \alpha_{2}, \beta_{2}\right) \\
& =\left(m_{1}+m_{2}, \max \left\{\alpha_{1}, \alpha_{2}\right\}, \max \left\{\beta_{1}, \beta_{2}\right\}\right), \\
\text { (2) } \widetilde{A}-\widetilde{B} & =\left(a_{1}, a_{2}, a_{3}\right)-\left(b_{1}, b_{2}, b_{3}\right) \\
& =\left(m_{1}, \alpha_{1}, \beta_{1}\right)-\left(m_{2}, \alpha_{2}, \beta_{2}\right) \\
& =\left(m_{1}-m_{2}, \max \left\{\alpha_{1}, \alpha_{2}\right\}, \max \left\{\beta_{1}, \beta_{2}\right\}\right), \\
\text { (3) } \widetilde{A} \widetilde{B}= & \left(a_{1}, a_{2}, a_{3}\right)\left(b_{1}, b_{2}, b_{3}\right) \\
= & \left(m_{1}, \alpha_{1}, \beta_{1}\right)\left(m_{2}, \alpha_{2}, \beta_{2}\right) \\
= & \left(m_{1} m_{2}, \max \left\{\alpha_{1}, \alpha_{2}\right\}, \max \left\{\beta_{1}, \beta_{2}\right\}\right),
\end{aligned}
$$

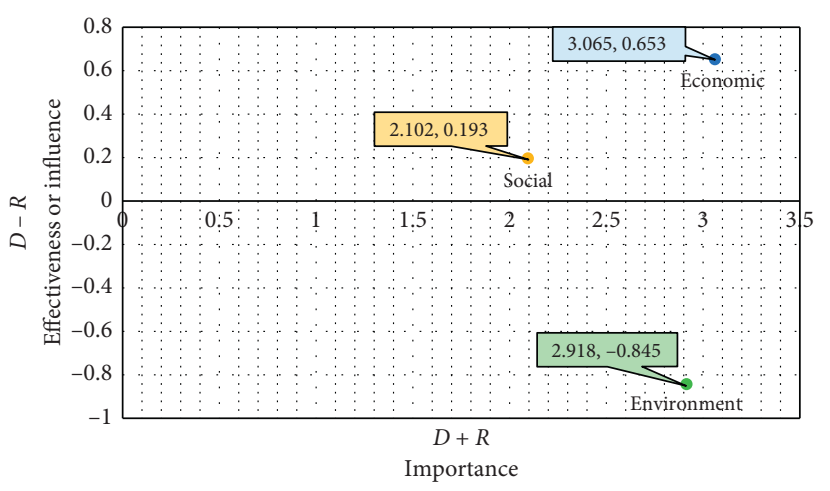

FIgURE 7: Effectiveness or influence and importance weight of sustainability dimensions.

$$
\text { (4) } \begin{aligned}
\frac{\tilde{A}}{\widetilde{B}} & =\frac{\left(a_{1}, a_{2}, a_{3}\right)}{\left(b_{1}, b_{2}, b_{3}\right)} \\
& =\frac{\left(m_{1}, \alpha_{1}, \beta_{1}\right)}{\left(m_{2}, \alpha_{2}, \beta_{2}\right)}=\left(\frac{m_{1}}{m_{2}}, \max \left\{\alpha_{1}, \alpha_{2}\right\}, \max \left\{\beta_{1}, \beta_{2}\right\}\right),
\end{aligned}
$$


TABLE 20: Comparison matrix of the linguistic variable of sustainability criteria in Z-number condition.

\begin{tabular}{|c|c|c|c|c|c|c|c|c|c|c|c|c|}
\hline & $E 1$ & E2 & E3 & $E 4$ & $S 1$ & $S 2$ & S3 & $S 4$ & G1 & G2 & G3 & G4 \\
\hline$E 1$ & & VH-C & $\mathrm{N}-\mathrm{U}$ & N-ML & VL-C & VL-L & VH-ML & L-L & VL-U & L-W & VL-W & $\mathrm{L}-\mathrm{U}$ \\
\hline$E 2$ & VH-L & & N-W & N-L & VL-W & VL-W & VH-FI & L-ML & VL-ML & $\mathrm{L}-\mathrm{C}$ & VL-M & L-M \\
\hline E3 & $\mathrm{N}-\mathrm{M}$ & N-M & & $\mathrm{N}-\mathrm{U}$ & VL-C & N-L & VH-W & L-C & N-FI & $\mathrm{N}-\mathrm{C}$ & $\mathrm{N}-\mathrm{U}$ & N-L \\
\hline$E 4$ & L-M & VL-ML & VL-L & & $\mathrm{L}-\mathrm{W}$ & N-FI & H-L & L-ML & H-M & $\mathrm{H}-\mathrm{C}$ & L-ML & L-L \\
\hline$S 1$ & N-ML & N-C & N-FI & $\mathrm{N}-\mathrm{W}$ & & VH-U & $\mathrm{H}-\mathrm{U}$ & H-FI & N-L & N-L & $\mathrm{N}-\mathrm{U}$ & $\mathrm{N}-\mathrm{W}$ \\
\hline$S 2$ & VL-W & N-W & N-ML & N-M & VL-ML & & VH-L & H-W & N-W & $\mathrm{N}-\mathrm{C}$ & N-ML & N-W \\
\hline S3 & L-FI & $\mathrm{L}-\mathrm{U}$ & H-FI & N-M & H-FI & H-W & & H-FI & $\mathrm{H}-\mathrm{C}$ & $\mathrm{L}-\mathrm{W}$ & H-M & L-M \\
\hline$S 4$ & VL-W & VL-M & N-L & $\mathrm{N}-\mathrm{W}$ & VL-M & VL-W & L-W & & N-M & N-ML & N-L & N-FI \\
\hline G1 & VL-M & N-L & $\mathrm{N}-\mathrm{C}$ & N-ML & VL-M & $\mathrm{H}-\mathrm{ML}$ & $\mathrm{H}-\mathrm{C}$ & N-ML & & VL-FI & VL-C & VL-U \\
\hline$G 2$ & L-L & L-L & $\mathrm{N}-\mathrm{M}$ & VL-ML & N-L & VH-C & VH-FI & L-FI & $\mathrm{H}-\mathrm{ML}$ & & L-FI & L-M \\
\hline G3 & L-C & $\mathrm{L}-\mathrm{U}$ & $\mathrm{N}-\mathrm{C}$ & N-C & $\mathrm{N}-\mathrm{U}$ & VH-FI & VH-ML & L-W & L-C & $\mathrm{N}-\mathrm{U}$ & & $\mathrm{N}-\mathrm{L}$ \\
\hline G4 & VL-W & N-M & $\mathrm{N}-\mathrm{C}$ & N-L & $\mathrm{N}-\mathrm{W}$ & $\mathrm{H}-\mathrm{M}$ & H-U & VL-C & $\mathrm{N}-\mathrm{C}$ & N-M & N-L & \\
\hline
\end{tabular}

TABLE 21: Importance weight of sustainability criteria in Z-number condition.

\begin{tabular}{|c|c|c|c|c|c|c|c|}
\hline Criteria & $\widetilde{D}_{\mathbf{i}}+\widetilde{R}_{\mathbf{i}}$ & $\widetilde{D}_{\mathbf{i}}-\widetilde{R}_{\mathbf{i}}$ & $\left(\widetilde{D}_{\mathbf{i}}+\widetilde{R}_{\mathbf{i}}\right)^{\text {def }}$ & $\left(\widetilde{D}_{\mathbf{i}}-\widetilde{R}_{\mathbf{i}}\right)^{\text {def }}$ & Influence & $\mathrm{W}_{\mathrm{i}}$ & $\mathrm{W}_{\mathrm{i}}^{\text {goal }}$ \\
\hline$E 1$ & $(0.659,1.731,4.902)$ & $(-1.989,0.166,2.254)$ & 2.256 & 0.149 & Influence & 0.094 & 0.109 \\
\hline E2 & $(0.580,1.498,4.544)$ & $(-1.618,0.343,2.346)$ & 2.030 & 0.353 & Influence & 0.086 & 0.099 \\
\hline E3 & $(0.240,0.559,2.971)$ & $(-1.277,0.132,1.454)$ & 1.082 & 0.110 & Influence & 0.045 & 0.052 \\
\hline E4 & $(0.458,1.183,4.393)$ & $(-0.719,1.080,3.216)$ & 1.804 & 1.164 & Influence & 0.089 & 0.103 \\
\hline$S 1$ & $(0.268,0.838,3.464)$ & $(-1.885,-0.278,1.311)$ & 1.352 & -0.282 & Be-influence & 0.058 & 0.045 \\
\hline$S 2$ & $(0.712,1.558,4.634)$ & $(-2.640,-0.653,1.282)$ & 2.116 & -0.666 & Be-influence & 0.092 & 0.072 \\
\hline$S 3$ & $(1.412,2.689,6.442)$ & $(-3.097,-0.635,1.932)$ & 3.308 & -0.609 & Be-influence & 0.140 & 0.109 \\
\hline$S 4$ & $(0.469,1.304,4.477)$ & $(-2.909,-0.748,1.098)$ & 1.888 & -0.827 & Be-influence & 0.086 & 0.067 \\
\hline$G 1$ & $(0.506,1.310,4.612)$ & $(-2.212,-0.130,1.894)$ & 1.935 & -0.144 & Be-influence & 0.081 & 0.090 \\
\hline G2 & $(0.683,1.587,4.907)$ & $(-1.498,0.605,2.725)$ & 2.191 & 0.609 & Influence & 0.095 & 0.106 \\
\hline G3 & $(0.546,1.303,4.254)$ & $(-1.575,0.276,2.134)$ & 1.852 & 0.278 & Influence & 0.078 & 0.087 \\
\hline G4 & $(0.285,0.791,3.439)$ & $(-1.691,-0.157,1.463)$ & 1.327 & -0.135 & Be-influence & 0.056 & 0.062 \\
\hline
\end{tabular}

provided that $\widetilde{B}=\left(b_{1}, b_{2}, b_{3}\right)$ is opposed to the $(\widetilde{0})$ fuzzy number.

The concept of Z-numbers is mostly used to model uncertain information [67]. And it is different from what is presented [68] as the Z-numbers. Their meaning and definition are shown as follows:

$$
Z=(A, B)=Z^{+}\left(A, \mu_{A} \cdot p_{X_{A}} \text { is } B\right) .
$$

This definition states that, for an arbitrary variable $X_{A}$, if $Z^{+}$is a number of $Z^{+}$- number [67] $p_{X_{A}}$, the probability of the random variable $X_{A}$ for the fuzzy set of $A$ is a function of the membership. The fuzzy set $A$ is defined as $\mu_{A}(x)$, so that $x \in X_{A}$ and $x \in R$, where $R$ is the real value range, and the function of the membership of the fuzzy set $B$ is defined as $\mu_{B}(x)$, where $x \in X_{B}$ and $x \in R$, where $R$ is the real value range. In addition, $\mu_{A} \cdot p_{X_{A}}$ is $B$ indicates $\mu_{B}\left(\int \mu_{A}(x)\right.$ - $\left.p_{X_{A}}(x) \mathrm{d} x\right)$. If $X_{A}$ is selected arbitrarily, the concept of $Z-$ number is explained as follows:

$$
Z=Z^{+}\left(A, \mu_{A} \cdot p_{X_{A}} \text { is } B\right) \text {. }
$$

A simple Z-number is shown in Figure 2. A number of articles that rely on the above definition of $Z$-numbers, such as the ZBWM method for evaluating the supplier [69], a combination of $Z$-numbers and Bayesian decision theory [70], Z-VIKOR to make decisions [71], SMAA model for decision support [71], DEA and neural network to assess supply chain sustainability [72], sample selection of portfolio

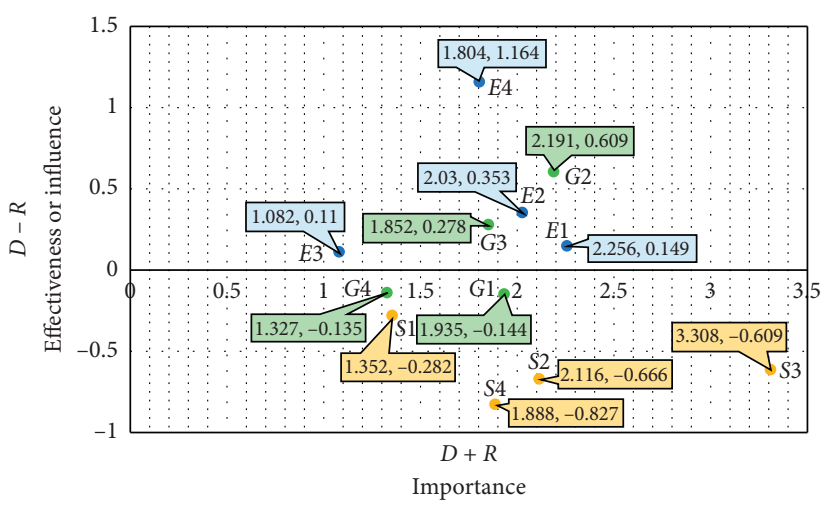

FIGURE 8: Effectiveness or influence and importance of sustainability criteria.

[73], and sustainable supplier selection by a possibilistic hierarchical model in the context of Z-information [74], have been studied.

Z-number is made up of three components with a $(X, A, B)$ structure. Obviously, due to the lack of accuracy and reliability of most data in various phenomena and problems in decision-making, data are associated with $Z$ information (i.e., there is a possibility of reliability), although they are not common in complex calculations. Therefore, to simplify the calculations and subsequently develop extensive applications of Z-number, a model for converting $Z$ - 
TABLE 22: Linguistic scoring variable matrix of sustainability criteria, considering the probability for proposed projects.

\begin{tabular}{lcccccccccccc}
\hline & $E 1$ & $E 2$ & $E 3$ & $E 4$ & $S 1$ & $S 2$ & $S 3$ & $S 4$ & $G 1$ & $G 2$ & $G 3$ & $G 4$ \\
\hline$P 1$ & $(1.235,1.3,1.365)$ & $(19.627,20.66,21.693)$ & $(26,29,33)$ & B-L & VS-U & M-L & VB-C & VS-L & VS-L & B-U & B-U & VB-L \\
$P 2$ & $(1.615,1.7,1.785)$ & $(23.902,25.16,26.418)$ & $(32,35,39)$ & B-ML & S-L & VS-W & B-M & M-C & VB-FI & VB- & B-ML & VS-L \\
P3 & $(0.827,0.87,0.914)$ & $(14.44,15.2,15.96)$ & $(14,17,21)$ & VS-L & VS-U & S-FI & VB- & VB-W & VB-C & M-L & VS- & M- \\
P4 & $(1.881,1.98,2.079)$ & $(25.365,26.7,28.035)$ & $(43,46,50)$ & B-ML & S-C & VS-L & VB-U & S-FI & M-U & S-FI & VB-M & B-L \\
P5 & $(1.064,1.12,1.176)$ & $(18.459,19.43,20.402)$ & $(21,24,28)$ & VS- & VS-M & S-W & S-ML & VS-ML & VS- & M-C & VS-L & VS-C \\
P6 & $(2.014,2.12,2.226)$ & $(28.595,30.1,31.605)$ & $(48,51,55)$ & B-ML & M- & M- & M-ML & M-U & B-M & VS-U & S-M & M-W \\
P7 & $(1.777,1.87,1.964)$ & $(22.582,23.77,24.959)$ & $(39,42,46)$ & VS-L & S-M & VS-FI & S-L & VB- & VS-W & M-W & B-U & B-L \\
P8 & $(0.931,0.98,1.029)$ & $(16.084,16.93,17.777)$ & $(16,19,23)$ & B-M & VB-C & VB-U & VB-FI & B-C & S-M & B-ML & VS- & B-ML \\
\hline
\end{tabular}

TABLE 23: Comparison matrix of linguistic variable of sustainability dimensions in deterministic condition.

\begin{tabular}{lccc}
\hline & Economic & Social & Environment \\
\hline Economic & 0.000 & 3.000 & 4.000 \\
Social & 1.000 & 0.000 & 2.000 \\
Environment & 3.000 & 0.000 & 0.000 \\
\hline
\end{tabular}

TABLE 24: Normalized matrix of sustainability dimensions in deterministic condition.

\begin{tabular}{lccc}
\hline & Economic & Social & Environment \\
\hline Economic & 0.000 & 0.429 & 0.571 \\
Social & 0.143 & 0.000 & 0.286 \\
Environment & 0.429 & 0.000 & 0.000 \\
\hline
\end{tabular}

numbers to conventional fuzzy sets has been provided [67]. For a pair of $Z$-number $(A, B)$, if $X$ is a random variable with a specific distribution, then $X$ becomes $A$, depicting a fuzzy event in $R$ with probability. This is shown as follows:

$$
p=\int_{R} \mu_{A}(u) p_{x}(u) d_{u}
$$

where $p_{x}$ is the probability distribution of $X$. As a constraint, the structure of $(X, A, B)$ in $X$ is expressed as follows [67]:

$$
\operatorname{Prob}(X \text { is } A) \text { is } B \text {. }
$$

Using equation (2), $\operatorname{Prob}(X$ is $A)$ is $B$ is replaced with $p=\int_{R} \mu_{A}(u) p_{x}(u) d_{u}$; then

$$
p=\int_{R} \mu_{A}(u) p_{x}(u) d_{u} \text { is } B .
$$

According to the probability rule, the complement of $Z$ is $Z^{\prime}$. For example, the complement of $(A, B)$ in $Z$-number is $\left(A^{\prime}, 1-B\right)$ (Figure 3).

The $\mathrm{Z}^{+}$-number concept, which is closely related to the concept of Z-numbers, is in fact a custom pair $(R, A)$ in which $A$ plays the same role as $Z$ and $R$ and is the same probability distribution. In addition, a $Z^{+}$-number can be defined as a pair $\left(\mu_{A}, p_{x}\right)$ in which $A$ plays the role of $A$ 's membership function and $p_{x}$ indicates the probability
TABLE 25: Total relationship matrix of sustainability dimensions in deterministic condition.

\begin{tabular}{lccc}
\hline & Economic & Social & Environment \\
\hline Economic & 0.559 & 0.668 & 1.082 \\
Social & 0.414 & 0.177 & 0.573 \\
Environment & 0.668 & 0.286 & 0.464 \\
\hline
\end{tabular}

TABLE 26: Importance weight of sustainability dimensions in deterministic condition.

\begin{tabular}{lcccc}
\hline Dimension & $\mathbf{D}_{\mathbf{i}}+\mathbf{R}_{\mathbf{i}}$ & $\mathbf{D}_{\mathbf{i}}-\mathbf{R}_{\mathbf{i}}$ & Influence & $\mathbf{W}_{\mathbf{i}}^{\text {goal }}$ \\
\hline Economic & 3.950 & 0.668 & Influence & 0.404 \\
Social & 2.295 & 0.032 & Influence & 0.232 \\
Environment & 3.536 & -0.700 & Be-influence & 0.364 \\
\hline
\end{tabular}

TABLE 27: Comparison matrix of linguistic variable of sustainability criteria in deterministic condition.

\begin{tabular}{ccccccccccccc}
\hline & $E 1$ & $E 2$ & $E 3$ & $E 4$ & $S 1$ & $S 2$ & $S 3$ & $S 4$ & $G 1$ & $G 2$ & $G 3$ & $G 4$ \\
\hline$C_{1}$ & 0 & 4 & 0 & 0 & 1 & 1 & 4 & 2 & 1 & 2 & 1 & 2 \\
$C_{2}$ & 4 & 0 & 0 & 0 & 1 & 1 & 4 & 2 & 1 & 2 & 1 & 2 \\
$C_{3}$ & 0 & 0 & 0 & 0 & 1 & 0 & 4 & 2 & 0 & 0 & 0 & 0 \\
$C_{4}$ & 2 & 1 & 1 & 0 & 2 & 0 & 3 & 2 & 3 & 3 & 2 & 2 \\
$C_{5}$ & 0 & 0 & 0 & 0 & 0 & 4 & 3 & 3 & 0 & 0 & 0 & 0 \\
$C_{6}$ & 1 & 0 & 0 & 0 & 1 & 0 & 4 & 3 & 0 & 0 & 0 & 0 \\
$C_{7}$ & 2 & 2 & 3 & 0 & 3 & 3 & 0 & 3 & 3 & 2 & 3 & 2 \\
$C_{8}$ & 1 & 1 & 0 & 0 & 1 & 1 & 2 & 0 & 0 & 0 & 0 & 0 \\
$C_{9}$ & 1 & 0 & 0 & 0 & 1 & 3 & 3 & 0 & 0 & 1 & 1 & 1 \\
$C_{10}$ & 2 & 2 & 0 & 1 & 0 & 4 & 4 & 2 & 3 & 0 & 2 & 2 \\
$C_{11}$ & 2 & 2 & 0 & 0 & 0 & 4 & 4 & 2 & 2 & 0 & 0 & 0 \\
$C_{12}$ & 1 & 0 & 0 & 0 & 0 & 3 & 3 & 1 & 0 & 0 & 0 & 0 \\
\hline
\end{tabular}

distribution of $X$. In fact, the difference between the $Z^{+}$number and $\mathrm{Z}$-number is the fact that in $\mathrm{Z}$-number, $p_{x}$ (probability distribution) is unknown. However, the probability value of $A$ is known [67]. The relationship between $\mathrm{Z}^{+}$-number and $\mathrm{Z}$-number is explained as follows:

$$
Z(A, B)=Z^{+}\left(A, \mu_{A} \cdot p_{X} \text { is } B\right)
$$

According to the DE fuzzy model provided by Kang et al., a specific Z-number with a pair 
TABLE 28: Normalized matrix of sustainability criteria in deterministic condition.

\begin{tabular}{lccccccccccccc}
\hline & $E 1$ & $E 2$ & $E 3$ & $E 4$ & $S 1$ & $S 2$ & $S 3$ & $S 4$ & $G 1$ & $G 2$ & $G 3$ \\
\hline$E 1$ & 0 & 0.154 & 0 & 0 & 0.038 & 0.038 & 0.154 & 0.077 & 0.038 & 0.077 & 0.038 & 0.077 \\
$E 2$ & 0.154 & 0 & 0 & 0 & 0.038 & 0.038 & 0.154 & 0.077 & 0.038 & 0.077 & 0.038 & 0.077 \\
$E 3$ & 0 & 0 & 0 & 0 & 0.038 & 0 & 0.154 & 0.077 & 0 & 0 & 0 & 0 \\
$E 4$ & 0.077 & 0.038 & 0.038 & 0 & 0.077 & 0 & 0.115 & 0.077 & 0.115 & 0.115 & 0.077 & 0.077 \\
$S 1$ & 0 & 0 & 0 & 0 & 0 & 0.154 & 0.115 & 0.115 & 0 & 0 & 0 & 0 \\
$S 2$ & 0.038 & 0 & 0 & 0 & 0.038 & 0 & 0.154 & 0.115 & 0 & 0 & 0 & 0 \\
$S 3$ & 0.077 & 0.077 & 0.115 & 0 & 0.115 & 0.115 & 0 & 0.115 & 0.115 & 0.077 & 0.115 & 0.077 \\
$S 4$ & 0.038 & 0.038 & 0 & 0 & 0.038 & 0.038 & 0.077 & 0 & 0 & 0 & 0 & 0 \\
$G 1$ & 0.038 & 0 & 0 & 0 & 0.038 & 0.115 & 0.115 & 0 & 0 & 0.038 & 0.038 & 0.038 \\
$G 2$ & 0.077 & 0.077 & 0 & 0.038 & 0 & 0.154 & 0.154 & 0.077 & 0.115 & 0 & 0.077 & 0.077 \\
$G 3$ & 0.077 & 0.077 & 0 & 0 & 0 & 0.154 & 0.154 & 0.077 & 0.077 & 0 & 0 & 0 \\
$G 4$ & 0.038 & 0 & 0 & 0 & 0 & 0.115 & 0.115 & 0.038 & 0 & 0 & 0 \\
\hline
\end{tabular}

TABLE 29: Total relationship matrix of sustainability criteria in deterministic condition.

\begin{tabular}{lcccccccccccc}
\hline & $E 1$ & $E 2$ & $E 3$ & $E 4$ & $S 1$ & $S 2$ & $S 3$ & $S 4$ & $G 1$ & $G 2$ & $G 3$ \\
\hline$E 1$ & 0.103 & 0.221 & 0.038 & 0.005 & 0.110 & 0.180 & 0.332 & 0.201 & 0.113 & 0.132 & 0.104 & 0.142 \\
$E 2$ & 0.236 & 0.088 & 0.038 & 0.005 & 0.110 & 0.180 & 0.332 & 0.201 & 0.113 & 0.132 & 0.104 & 0.142 \\
$E 3$ & 0.034 & 0.030 & 0.024 & 0.001 & 0.074 & 0.057 & 0.209 & 0.128 & 0.032 & 0.022 & 0.030 & 0.024 \\
$E 4$ & 0.173 & 0.123 & 0.076 & 0.007 & 0.151 & 0.168 & 0.321 & 0.210 & 0.196 & 0.171 & 0.146 & 0.146 \\
$S 1$ & 0.041 & 0.032 & 0.022 & 0.001 & 0.042 & 0.206 & 0.194 & 0.179 & 0.030 & 0.022 & 0.028 & 0.023 \\
$S 2$ & 0.079 & 0.041 & 0.026 & 0.001 & 0.081 & 0.068 & 0.227 & 0.176 & 0.037 & 0.028 & 0.035 & 0.030 \\
$S 3$ & 0.177 & 0.156 & 0.144 & 0.005 & 0.190 & 0.280 & 0.243 & 0.263 & 0.185 & 0.129 & 0.174 & 0.139 \\
$S 4$ & 0.07 & 0.065 & 0.016 & 0.001 & 0.066 & 0.084 & 0.137 & 0.049 & 0.026 & 0.022 & 0.024 & 0.024 \\
$G 1$ & 0.089 & 0.045 & 0.026 & 0.003 & 0.083 & 0.198 & 0.223 & 0.085 & 0.045 & 0.068 & 0.077 & 0.073 \\
$G 2$ & 0.182 & 0.159 & 0.044 & 0.041 & 0.087 & 0.303 & 0.364 & 0.219 & 0.194 & 0.066 & 0.148 & 0.147 \\
$G 3$ & 0.155 & 0.0 & 0.035 & 0.002 & 0.070 & 0.257 & 0.305 & 0.186 & 0.134 & 0.051 & 0.056 & 0.055 \\
$G 4$ & 0.075 & 0.034 & 0.022 & 0.001 & 0.038 & 0.166 & 0.188 & 0.099 & 0.031 & 0.024 & 0.029 & 0.026 \\
\hline
\end{tabular}

$Z=(A, B), \widetilde{B}=\left\{\left(x, \mu_{\widetilde{B}}(x) \mid x \varepsilon[0,1]\right\}\right.$ indicates the fuzzy reliability value and $\mu_{B}(x)$ indicates the membership function. The value of the center of gravity (central defuzzy) is obtained according to the following equation:

$$
\alpha=\frac{\int x \mu_{B}^{\sim}(x) \mathrm{d}_{x}}{\int \mu_{B}(x) \mathrm{d}_{x}} .
$$

If $\widetilde{B} \sim \operatorname{TrFS}[a, b, c]$, then the center of gravity of this set is $a+b+c / 3$. If $\bar{A} \sim \operatorname{TrFS}[d, e, f]$ converted into Z-number, the following equation will appear:

$$
A^{\prime}=[\sqrt{\alpha} d, \sqrt{\alpha} e, \sqrt{\alpha} f]=\left[d^{\prime}, e^{\prime}, f^{\prime}\right] .
$$

The following statement is inferred using the defuzzy method. Figure 4 (a) shows a situation where $A^{\prime}<A$. This situation indicates that some (or only one component) components are less likely than 1, for example, $([2,4,7,9.5],[0.8,0.8,0.9,1]) . \alpha$ obtained by equations (3)-(6) is less than $1.00(\alpha<1)$ and the number $Z$ is less than its fuzzy state. Unlike the previous case, there are conditions where all the components are deterministic and their probability is 1.00 . For example, $([2,4,7,9.5],[1,1,1,1])$. In such cases, the number $Z$ may be defined as a simple fuzzy set $(\alpha \leq 1)$ (Figure $4(b))$ [75].

In the present study, the probability is taken into consideration for each fuzzy number by $Z$-numbers. Since linguistic variables do not always come with certainty, possibilities like "unlikely" and "weak" have to be considered
TABLE 30: Importance weight of sustainability criteria in deterministic condition.

\begin{tabular}{lccccc}
\hline Criteria & $\left(\widetilde{D}_{\mathbf{i}}+\widetilde{R}_{\mathbf{i}}\right)^{\text {def }}$ & $\left(\widetilde{D}_{\mathbf{i}}-\widetilde{R}_{\mathbf{i}}\right)^{\text {def }}$ & Influence & $\mathbf{W}_{\mathbf{i}}$ & $\mathbf{W}_{\mathbf{i}}^{\text {goal }}$ \\
\hline E1 & 3.094 & 0.269 & Influence & 0.095 & 0.120 \\
$E 2$ & 2.816 & 0.546 & Influence & 0.088 & 0.111 \\
E3 & 1.178 & 0.155 & Influence & 0.036 & 0.046 \\
E4 & 1.958 & 1.814 & Influence & 0.082 & 0.103 \\
$S 1$ & 1.924 & -0.284 & Be-influence & 0.060 & 0.043 \\
S2 & 2.976 & -1.315 & Be-influence & 0.100 & 0.072 \\
$S 3$ & 5.158 & -0.991 & Be-influence & 0.161 & 0.116 \\
$S 4$ & 2.578 & -1.410 & Be-influence & 0.090 & 0.065 \\
$G 1$ & 2.149 & -0.120 & Be-influence & 0.066 & 0.075 \\
$G 2$ & 2.824 & 1.086 & Influence & 0.093 & 0.105 \\
$G 3$ & 2.399 & 0.492 & Influence & 0.075 & 0.085 \\
$G 4$ & 1.703 & -0.241 & Be-influence & 0.053 & 0.060 \\
\hline
\end{tabular}

Table 31: Decision matrix in deterministic condition.

\begin{tabular}{ccccccccccccc}
\hline & $E 1$ & $E 2$ & $E 3$ & $E 4$ & $S 1$ & $S 2$ & $S 3$ & $S 4$ & $G 1$ & $G 2$ & $G 3$ & $G 4$ \\
\hline$P 1$ & 1.3 & 20.66 & 29 & 4 & 1 & 3 & 5 & 1 & 1 & 4 & 4 & 5 \\
$P 2$ & 1.7 & 25.16 & 35 & 4 & 2 & 1 & 4 & 3 & 5 & 5 & 4 & 1 \\
$P 3$ & 0.87 & 15.2 & 17 & 1 & 1 & 2 & 5 & 5 & 5 & 3 & 1 & 3 \\
$P 4$ & 1.98 & 26.7 & 46 & 4 & 2 & 1 & 5 & 2 & 3 & 2 & 5 & 4 \\
$P 5$ & 1.12 & 19.43 & 24 & 1 & 1 & 2 & 2 & 1 & 1 & 3 & 1 & 1 \\
$P 6$ & 2.12 & 30.1 & 51 & 4 & 3 & 3 & 3 & 3 & 4 & 1 & 2 & 3 \\
$P 7$ & 1.87 & 23.77 & 42 & 1 & 2 & 1 & 2 & 5 & 1 & 3 & 4 & 4 \\
$P 8$ & 0.98 & 16.93 & 19 & 4 & 5 & 5 & 5 & 4 & 2 & 4 & 1 & 4 \\
\hline
\end{tabular}


TABLE 32: Normalized decision matrix in deterministic condition.

\begin{tabular}{lcccccccccccc}
\hline \multirow{2}{*}{ Weight } & 0.12 & 0.111 & 0.046 & 0.103 & 0.043 & 0.072 & 0.116 & 0.065 & 0.075 & 0.105 & 0.085 & 0.06 \\
& $E 1$ & $E 2$ & $E 3$ & $E 4$ & $S 1$ & $S 2$ & $S 3$ & $S 4$ & $G 1$ & $G 2$ \\
\hline$P 1$ & 0.656 & 0.634 & 0.353 & 0 & 1 & 0.5 & 0 & 1 & 0 & 0.25 & 0.75 \\
$P 2$ & 0.336 & 0.332 & 0.529 & 0 & 0.75 & 1 & 0.333 & 0.5 & 1 & 0 & 0.75 & 1 \\
$P 3$ & 1 & 1 & 0 & 1 & 1 & 0.75 & 0 & 0 & 1 & 0.5 & 0 & 0.5 \\
$P 4$ & 0.112 & 0.228 & 0.853 & 0 & 0.75 & 1 & 0 & 0.75 & 0.5 & 0.75 & 1 & 0.25 \\
$P 5$ & 0.8 & 0.716 & 0.206 & 1 & 1 & 0.75 & 1 & 1 & 0 & 0.5 & 0 & 1 \\
$P 6$ & 0 & 0 & 1 & 0 & 0.5 & 0.5 & 0.667 & 0.5 & 0.75 & 1 & 0.25 & 0.5 \\
$P 7$ & 0.2 & 0.425 & 0.735 & 1 & 0.75 & 1 & 1 & 0 & 0 & 0.5 & 0.75 & 0.25 \\
$P 8$ & 0.912 & 0.884 & 0.059 & 0 & 0 & 0 & 0 & 0.25 & 0.25 & 0.25 & 0 & 0.25 \\
\hline
\end{tabular}

TABLE 33: Project ranking in deterministic condition.

\begin{tabular}{|c|c|c|c|c|c|c|c|}
\hline \multirow{2}{*}{ Project } & \multirow{2}{*}{$S$} & \multirow{2}{*}{$R$} & \multirow{2}{*}{$Q$} & \multicolumn{3}{|c|}{ Rank by } & \multirow{2}{*}{ Final rank } \\
\hline & & & & $R$ & $S$ & $Q$ & \\
\hline$P 1$ & 0.399 & 0.079 & 0.185 & 2 & 2 & 1 & 1 \\
\hline$P 2$ & 0.476 & 0.075 & 0.241 & 1 & 5 & 2 & 2 \\
\hline$P 3$ & 0.589 & 0.12 & 0.885 & 8 & 7 & 7 & $x$ \\
\hline$P 4$ & 0.447 & 0.085 & 0.316 & 3 & 4 & 3 & $x$ \\
\hline$P 5$ & 0.678 & 0.116 & 0.956 & 6 & 8 & 8 & $x$ \\
\hline$P 6$ & 0.426 & 0.105 & 0.511 & 4 & 3 & 5 & $x$ \\
\hline P7 & 0.559 & 0.116 & 0.804 & 6 & 6 & 6 & $x$ \\
\hline$P 8$ & 0.287 & 0.109 & 0.383 & 5 & 1 & 4 & $x$ \\
\hline
\end{tabular}

TABLE 34: Comparison matrix of linguistic variable of sustainability dimensions in fuzzy condition.

\begin{tabular}{lcrr}
\hline & Economic & Social & Environment \\
\hline Economic & $(0.000,0.000,0.000)$ & $(0.500,0.750,1.000)$ & $(0.750,1.000,1.000)$ \\
Social & $(0.000,0.250,0.500)$ & $(0.000,0.000,0.000)$ & $(0.250,0.500,0.750)$ \\
Environment & $(0.500,0.750,1.000)$ & $(0.000,0.000,0.250)$ & $(0.000,0.000,0.000)$ \\
\hline
\end{tabular}

TABLE 35: Normalized matrix of sustainability dimensions in fuzzy condition.

\begin{tabular}{lccr}
\hline & Economic & Social & Environment \\
\hline Economic & $(0.000,0.000,0.000)$ & $(0.250,0.375,0.500)$ & $(0.375,0.500,0.500)$ \\
Social & $(0.000,0.125,0.250)$ & $(0.000,0.000,0.000)$ & $(0.125,0.250,0.375)$ \\
Environment & $(0.250,0.375,0.500)$ & $(0.000,0.000,0.125)$ & $(0.000,0.000,0.000)$ \\
\hline
\end{tabular}

TABLE 36: Total relationship matrix of sustainability dimensions in fuzzy condition.

\begin{tabular}{lccr}
\hline & Economic & Social & Environment \\
\hline Economic & $(0.113,0.369,1.033)$ & $(0.278,0.513,1.200)$ & $(0.452,0.813,1.467)$ \\
Social & $(0.035,0.299,0.933)$ & $(0.009,0.112,0.600)$ & $(0.139,0.428,1.067)$ \\
Environment & $(0.278,0.513,1.133)$ & $(0.070,0.193,0.800)$ & $(0.113,0.305,0.867)$ \\
\hline
\end{tabular}

TABLE 37: Importance weight of sustainability dimensions in fuzzy condition.

\begin{tabular}{|c|c|c|c|c|c|c|}
\hline Dimension & $\widetilde{D}_{\mathbf{i}}+\widetilde{R}_{\mathbf{i}}$ & $\left(\widetilde{D}_{\mathbf{i}}+\widetilde{R}_{\mathbf{i}}\right)^{\text {def }}$ & $\widetilde{D}_{\mathbf{i}}-\widetilde{R}_{\mathbf{i}}$ & $\left(\widetilde{D}_{\mathbf{i}}-\widetilde{R}_{\mathbf{i}}\right)^{\text {def }}$ & Influence & $\mathrm{W}_{\mathrm{i}}^{\text {goal }}$ \\
\hline Economic & $(1.270,2.877,6.800)$ & 3.456 & $(-2.257,0.513,3.274)$ & 0.511 & Influence & 0.392 \\
\hline Social & $(0.539,1.658,5.200)$ & 2.264 & $(-2.417,0.021,2.243)$ & -0.033 & Be-influence & 0.254 \\
\hline Environment & $(1.165,2.556,6.200)$ & 3.119 & $(-2.939,-0.535,2.096)$ & -0.478 & Be-influence & 0.354 \\
\hline
\end{tabular}

to obtain more precise results. These probabilities are also determined by the experts' opinions for the linguistic variables in Table 2. Subsequently, to be calculable, each linguistic probability is related to the corresponding possibility shown in Table 3. Table 3 has been presented by Hendiani and Bagherpour [76]. 
TABLE 38: Comparison matrix of linguistic variable of sustainability criteria in fuzzy condition.

\begin{tabular}{|c|c|c|c|c|c|c|c|c|c|c|c|c|}
\hline & $E 1$ & $E 2$ & E3 & $E 4$ & $S 1$ & $S 2$ & S3 & S4 & G1 & G2 & G3 & G4 \\
\hline$E 1$ & $\begin{array}{l}(0.000, \\
0.000, \\
0.000)\end{array}$ & $\begin{array}{l}(0.750, \\
1.000, \\
1.000)\end{array}$ & $\begin{array}{c}(0.000 \\
0.000 \\
0.250)\end{array}$ & $\begin{array}{l}(0.000 \\
0.000, \\
0.250)\end{array}$ & $\begin{array}{l}(0.000 \\
0.250 \\
0.500)\end{array}$ & $\begin{array}{l}(0.000, \\
0.250, \\
0.500)\end{array}$ & $\begin{array}{l}(0.750 \\
1.000 \\
1.000)\end{array}$ & $\begin{array}{l}(0.250 \\
0.500 \\
0.750)\end{array}$ & $\begin{array}{l}(0.000, \\
0.250, \\
0.500)\end{array}$ & $\begin{array}{c}(0.250 \\
0.500 \\
0.750)\end{array}$ & $\begin{array}{c}(0.000, \\
0.250, \\
0.500)\end{array}$ & $\begin{array}{l}(0.250 \\
0.500 \\
0.750)\end{array}$ \\
\hline$E 2$ & $\begin{array}{l}(0.750 \\
1.000 \\
1.000)\end{array}$ & $\begin{array}{l}(0.000 \\
0.000 \\
0.000)\end{array}$ & $\begin{array}{c}(0.000, \\
0.000, \\
0.250)\end{array}$ & $\begin{array}{l}(0.000 \\
0.000 \\
0.250)\end{array}$ & $\begin{array}{l}(0.000 \\
0.250 \\
0.500)\end{array}$ & $\begin{array}{l}(0.000 \\
0.250 \\
0.500)\end{array}$ & $\begin{array}{l}(0.750 \\
1.000 \\
1.000)\end{array}$ & $\begin{array}{l}(0.250 \\
0.500 \\
0.750)\end{array}$ & $\begin{array}{c}(0.000 \\
0.250 \\
0.500)\end{array}$ & $\begin{array}{l}(0.250 \\
0.500 \\
0.750)\end{array}$ & $\begin{array}{c}(0.000, \\
0.250, \\
0.500)\end{array}$ & $\begin{array}{c}(0.250 \\
0.500 \\
0.750)\end{array}$ \\
\hline$E 3$ & $\begin{array}{l}(0.000, \\
0.000, \\
0.250)\end{array}$ & $\begin{array}{l}(0.000 \\
0.000 \\
0.250)\end{array}$ & $\begin{array}{c}(0.000, \\
0.000, \\
0.000)\end{array}$ & $\begin{array}{l}(0.000 \\
0.000 \\
0.250)\end{array}$ & $\begin{array}{c}(0.000, \\
0.250, \\
0.500)\end{array}$ & $\begin{array}{l}(0.000, \\
0.000 \\
0.250)\end{array}$ & $\begin{array}{l}(0.750, \\
1.000 \\
1.000)\end{array}$ & $\begin{array}{l}(0.250 \\
0.500 \\
0.750)\end{array}$ & $\begin{array}{l}(0.000 \\
0.000 \\
0.250)\end{array}$ & $\begin{array}{l}(0.000 \\
0.000 \\
0.250)\end{array}$ & $\begin{array}{l}(0.000, \\
0.000, \\
0.250)\end{array}$ & $\begin{array}{l}(0.000 \\
0.000 \\
0.250)\end{array}$ \\
\hline$E 4$ & $\begin{array}{l}(0.250, \\
0.500 \\
0.750)\end{array}$ & $\begin{array}{l}(0.000 \\
0.250 \\
0.500)\end{array}$ & $\begin{array}{l}(0.000, \\
0.250, \\
0.500)\end{array}$ & $\begin{array}{l}(0.000 \\
0.000 \\
0.000)\end{array}$ & $\begin{array}{l}(0.250, \\
0.500, \\
0.750)\end{array}$ & $\begin{array}{l}(0.000, \\
0.000 \\
0.250)\end{array}$ & $\begin{array}{l}(0.500, \\
0.750, \\
1.000)\end{array}$ & $\begin{array}{l}(0.250 \\
0.500 \\
0.750)\end{array}$ & $\begin{array}{l}(0.500, \\
0.750, \\
1.000)\end{array}$ & $\begin{array}{l}(0.500, \\
0.750, \\
1.000)\end{array}$ & $\begin{array}{l}(0.250, \\
0.500, \\
0.750)\end{array}$ & $\begin{array}{l}(0.250, \\
0.500, \\
0.750)\end{array}$ \\
\hline$S 1$ & $\begin{array}{l}(0.000, \\
0.000, \\
0.250)\end{array}$ & $\begin{array}{l}(0.000 \\
0.000 \\
0.250)\end{array}$ & $\begin{array}{l}(0.000, \\
0.000, \\
0.250)\end{array}$ & $\begin{array}{l}(0.000 \\
0.000 \\
0.250)\end{array}$ & $\begin{array}{l}(0.000, \\
0.000, \\
0.000)\end{array}$ & $\begin{array}{l}(0.750, \\
1.000 \\
1.000)\end{array}$ & $\begin{array}{l}(0.500, \\
0.750 \\
1.000)\end{array}$ & $\begin{array}{l}(0.500 \\
0.750 \\
1.000)\end{array}$ & $\begin{array}{l}(0.000 \\
0.000 \\
0.250)\end{array}$ & $\begin{array}{c}(0.000 \\
0.000 \\
0.250)\end{array}$ & $\begin{array}{l}(0.000, \\
0.000, \\
0.250)\end{array}$ & $\begin{array}{l}(0.000, \\
0.000, \\
0.250)\end{array}$ \\
\hline$S 2$ & $\begin{array}{l}(0.000, \\
0.250, \\
0.500)\end{array}$ & $\begin{array}{l}(0.000 \\
0.000 \\
0.250)\end{array}$ & $\begin{array}{l}(0.000, \\
0.000, \\
0.250)\end{array}$ & $\begin{array}{l}(0.000 \\
0.000 \\
0.250)\end{array}$ & $\begin{array}{l}(0.000, \\
0.250, \\
0.500)\end{array}$ & $\begin{array}{l}(0.000, \\
0.000, \\
0.000)\end{array}$ & $\begin{array}{l}(0.750, \\
1.000, \\
1.000)\end{array}$ & $\begin{array}{l}(0.500, \\
0.750 \\
1.000)\end{array}$ & $\begin{array}{l}(0.000, \\
0.000, \\
0.250)\end{array}$ & $\begin{array}{l}(0.000, \\
0.000, \\
0.250)\end{array}$ & $\begin{array}{l}(0.000, \\
0.000, \\
0.250)\end{array}$ & $\begin{array}{l}(0.000, \\
0.000, \\
0.250)\end{array}$ \\
\hline$S 3$ & & $\begin{array}{l}(0.250 \\
0.500 \\
0.750)\end{array}$ & $\begin{array}{c}(0.500, \\
0.750, \\
1.000)\end{array}$ & $\begin{array}{l}(0.000 \\
0.000 \\
0.250)\end{array}$ & $\begin{array}{l}(0.500, \\
0.750, \\
1.000)\end{array}$ & $\begin{array}{l}(0.500, \\
0.750, \\
1.000)\end{array}$ & $\begin{array}{l}(0.000 \\
0.000 \\
0.000)\end{array}$ & $\begin{array}{l}(0.500 \\
0.750 \\
1.000)\end{array}$ & $\begin{array}{c}(0.500, \\
0.750, \\
1.000)\end{array}$ & $\begin{array}{l}(0.250 \\
0.500 \\
0.750)\end{array}$ & $\begin{array}{l}(0.500, \\
0.750, \\
1.000)\end{array}$ & $\begin{array}{c}(0.250, \\
0.500, \\
0.750)\end{array}$ \\
\hline$S 4$ & $\begin{array}{l}(0.000, \\
0.250 \\
0.500)\end{array}$ & $\begin{array}{l}(0.000 \\
0.250 \\
0.500)\end{array}$ & $\begin{array}{l}(0.000, \\
0.000, \\
0.250)\end{array}$ & $\begin{array}{l}(0.000 \\
0.000 \\
0.250)\end{array}$ & $\begin{array}{l}(0.000, \\
0.250, \\
0.500)\end{array}$ & $\begin{array}{l}(0.000, \\
0.250 \\
0.500)\end{array}$ & $\begin{array}{l}(0.250 \\
0.500 \\
0.750)\end{array}$ & $\begin{array}{l}(0.000 \\
0.000 \\
0.000)\end{array}$ & $\begin{array}{c}(0.000, \\
0.000, \\
0.250)\end{array}$ & $\begin{array}{l}(0.000, \\
0.000, \\
0.250)\end{array}$ & $\begin{array}{c}(0.000, \\
0.000, \\
0.250)\end{array}$ & $\begin{array}{c}(0.000, \\
0.000, \\
0.250)\end{array}$ \\
\hline$G 1$ & $\begin{array}{c}(0.000, \\
0.250, \\
0.500)\end{array}$ & $\begin{array}{l}(0.000, \\
0.000, \\
0.250)\end{array}$ & $\begin{array}{c}(0.000, \\
0.000, \\
0.250)\end{array}$ & $\begin{array}{l}(0.000, \\
0.000, \\
0.250)\end{array}$ & $\begin{array}{c}(0.000, \\
0.250, \\
0.500)\end{array}$ & $\begin{array}{l}(0.500, \\
0.750 \\
1.000)\end{array}$ & $\begin{array}{l}(0.500, \\
0.750 \\
1.000)\end{array}$ & $\begin{array}{l}(0.000 \\
0.000 \\
0.250)\end{array}$ & $\begin{array}{c}(0.000, \\
0.000, \\
0.000)\end{array}$ & $\begin{array}{l}(0.000, \\
0.250 \\
0.500)\end{array}$ & $\begin{array}{c}(0.000, \\
0.250, \\
0.500)\end{array}$ & $\begin{array}{c}(0.000, \\
0.250, \\
0.500)\end{array}$ \\
\hline G2 & $\begin{array}{c}(0.250, \\
0.500, \\
0.750)\end{array}$ & $\begin{array}{c}(0.250 \\
0.500 \\
0.750)\end{array}$ & $\begin{array}{c}(0.000, \\
0.000, \\
0.250)\end{array}$ & $\begin{array}{c}(0.000, \\
0.250, \\
0.500)\end{array}$ & $\begin{array}{c}(0.000, \\
0.000, \\
0.250)\end{array}$ & $\begin{array}{c}(0.750, \\
1.000, \\
1.000)\end{array}$ & $\begin{array}{l}(0.750, \\
1.000, \\
1.000)\end{array}$ & $\begin{array}{l}(0.250, \\
0.500, \\
0.750)\end{array}$ & $\begin{array}{c}(0.500, \\
0.750, \\
1.000)\end{array}$ & $\begin{array}{l}(0.000, \\
0.000, \\
0.000)\end{array}$ & $\begin{array}{c}(0.250, \\
0.500, \\
0.750)\end{array}$ & $\begin{array}{c}(0.250, \\
0.500, \\
0.750)\end{array}$ \\
\hline G3 & $\begin{array}{l}(0.250, \\
0.500, \\
0.750)\end{array}$ & $\begin{array}{l}(0.250, \\
0.500, \\
0.750)\end{array}$ & $\begin{array}{l}(0.000, \\
0.000, \\
0.250)\end{array}$ & $\begin{array}{l}(0.000, \\
0.000, \\
0.250)\end{array}$ & $\begin{array}{l}(0.000, \\
0.000, \\
0.250)\end{array}$ & $\begin{array}{l}(0.750, \\
1.000, \\
1.000)\end{array}$ & $\begin{array}{c}(0.750, \\
1.000, \\
1.000)\end{array}$ & $\begin{array}{l}(0.250, \\
0.500, \\
0.750)\end{array}$ & $\begin{array}{c}(0.250, \\
0.500, \\
0.750)\end{array}$ & $\begin{array}{l}(0.000, \\
0.000, \\
0.250)\end{array}$ & $\begin{array}{l}(0.000, \\
0.000, \\
0.000)\end{array}$ & $\begin{array}{l}(0.000, \\
0.000, \\
0.250)\end{array}$ \\
\hline G4 & $\begin{array}{l}(0.000, \\
0.250, \\
0.500)\end{array}$ & $\begin{array}{l}(0.000, \\
0.000, \\
0.250)\end{array}$ & $\begin{array}{l}(0.000, \\
0.000, \\
0.250)\end{array}$ & $\begin{array}{l}(0.000, \\
0.000, \\
0.250)\end{array}$ & $\begin{array}{l}(0.000, \\
0.000, \\
0.250)\end{array}$ & $\begin{array}{l}(0.500, \\
0.750, \\
1.000)\end{array}$ & $\begin{array}{c}(0.500, \\
0.750, \\
1.000)\end{array}$ & $\begin{array}{l}(0.000, \\
0.250, \\
0.500)\end{array}$ & $\begin{array}{l}(0.000, \\
0.000, \\
0.250)\end{array}$ & $\begin{array}{l}(0.000, \\
0.000 \\
0.250)\end{array}$ & $\begin{array}{c}(0.000, \\
0.000, \\
0.250)\end{array}$ & $\begin{array}{l}(0.000, \\
0.000, \\
0.000)\end{array}$ \\
\hline
\end{tabular}

\section{Methodology}

This research is divided into four main steps as follows:

(i) Step 1: identification, classification, and filtering sustainability criteria to select sustainable projects in the relevant field using the Fernandez Sanchez and Rodriguez Lopez approach

(ii) Step 2: introducing the Z-number approach and the probabilistic linguistic variable

(iii) Step 3: introducing the Z-DEMATEL approach to evaluate the impact of the criteria on each other and their weighting and defining a new range of words for Z-number to be used in the pairwise comparison matrix to examine the impact of the sustainability criteria on each other

(iv) Step 4: introducing the Z-VIKOR approach for ranking sustainable projects and defining a new linguistic variable of $\mathrm{Z}$-number to be used in the decision-making matrix for scoring projects based on sustainability criteria
Figure 5 describes the research method process.

3.1. Identifying Sustainability Criteria for Project Selection. Human behavior is often at odds with the principles of sustainability. Continuation of these behaviors has many adverse effects on ecosystems and endangers the lives of future generations. Leaders and managers of the world must be aware of the bitter realities of the environment and expand and deepen those insights into the heart of societies, manage resources properly, stop the endless thirst for consumption, and plunder the limited natural resources. Challenges facing humanity today, such as economic, environmental, and social issues, are among the major challenges of the new century. To solve these problems, sustainable development has been proposed by experts, and hence, the need for different sciences to pay attention to this category is felt [77]. As mentioned earlier, companies are integrating sustainability ideas into their marketing, organizational communications, annual reports, and actions. Many of these actions are organized in projects which are the tools of change in organizations 
TABLE 39: Normalized matrix of sustainability criteria in fuzzy condition.

\begin{tabular}{|c|c|c|c|c|c|c|c|c|c|c|c|c|}
\hline & $E 1$ & $E 2$ & E3 & $E 4$ & $S 1$ & $S 2$ & $S 3$ & $S 4$ & $G 1$ & G2 & G3 & G4 \\
\hline$E 1$ & $\begin{array}{c}(0.000 \\
0.000 \\
0.000)\end{array}$ & $\begin{array}{c}(0.081, \\
0.108, \\
0.108)\end{array}$ & $\begin{array}{c}(0.000 \\
0.000 \\
0.027)\end{array}$ & $\begin{array}{l}(0.000 \\
0.000 \\
0.027)\end{array}$ & $\begin{array}{l}(0.000 \\
0.027 \\
0.054)\end{array}$ & $\begin{array}{l}(0.000 \\
0.027 \\
0.054)\end{array}$ & $\begin{array}{l}(0.081 \\
0.108 \\
0.108)\end{array}$ & $\begin{array}{l}(0.027, \\
0.054, \\
0.081)\end{array}$ & $\begin{array}{l}(0.000 \\
0.027 \\
0.054)\end{array}$ & $\begin{array}{c}(0.027 \\
0.054 \\
0.081)\end{array}$ & $\begin{array}{l}(0.000, \\
0.027 \\
0.054)\end{array}$ & $\begin{array}{c}(0.027, \\
0.054, \\
0.081)\end{array}$ \\
\hline E2 & $\begin{array}{c}(0.081 \\
0.108 \\
0.108)\end{array}$ & $\begin{array}{l}(0.000, \\
0.000, \\
0.000)\end{array}$ & $\begin{array}{l}(0.000 \\
0.000 \\
0.027)\end{array}$ & $\begin{array}{l}(0.000 \\
0.000 \\
0.027)\end{array}$ & $\begin{array}{c}(0.000, \\
0.027 \\
0.054)\end{array}$ & $\begin{array}{l}(0.000 \\
0.027 \\
0.054)\end{array}$ & $\begin{array}{l}(0.081 \\
0.108, \\
0.108)\end{array}$ & $\begin{array}{l}(0.027 \\
0.054 \\
0.081)\end{array}$ & $\begin{array}{l}(0.000, \\
0.027 \\
0.054)\end{array}$ & $\begin{array}{l}(0.027 \\
0.054 \\
0.081)\end{array}$ & $\begin{array}{l}(0.000 \\
0.027 \\
0.054)\end{array}$ & $\begin{array}{c}(0.027, \\
0.054, \\
0.081)\end{array}$ \\
\hline E3 & $\begin{array}{l}(0.000 \\
0.000 \\
0.027)\end{array}$ & $\begin{array}{l}(0.000, \\
0.000, \\
0.027)\end{array}$ & $\begin{array}{l}(0.000 \\
0.000 \\
0.000)\end{array}$ & $\begin{array}{l}(0.000 \\
0.000 \\
0.027)\end{array}$ & $\begin{array}{l}(0.000 \\
0.027 \\
0.054)\end{array}$ & $\begin{array}{l}(0.000 \\
0.000 \\
0.027)\end{array}$ & $\begin{array}{l}(0.081 \\
0.108, \\
0.108)\end{array}$ & $\begin{array}{l}(0.027, \\
0.054, \\
0.081)\end{array}$ & $\begin{array}{l}(0.000 \\
0.000 \\
0.027)\end{array}$ & $\begin{array}{c}(0.000 \\
0.000 \\
0.027)\end{array}$ & $\begin{array}{l}(0.000 \\
0.000 \\
0.027)\end{array}$ & $\begin{array}{c}(0.000, \\
0.000, \\
0.027)\end{array}$ \\
\hline$E 4$ & $\begin{array}{l}(0.027 \\
0.054 \\
0.081)\end{array}$ & $\begin{array}{l}(0.000, \\
0.027, \\
0.054)\end{array}$ & $\begin{array}{l}(0.000 \\
0.027 \\
0.054)\end{array}$ & $\begin{array}{l}(0.000, \\
0.000, \\
0.000)\end{array}$ & $\begin{array}{c}(0.027, \\
0.054, \\
0.081)\end{array}$ & $\begin{array}{l}(0.000 \\
0.000 \\
0.027)\end{array}$ & $\begin{array}{c}(0.054, \\
0.081, \\
0.108)\end{array}$ & $\begin{array}{l}(0.027, \\
0.054, \\
0.081)\end{array}$ & $\begin{array}{c}(0.054, \\
0.081, \\
0.108)\end{array}$ & $\begin{array}{c}(0.054 \\
0.081, \\
0.108)\end{array}$ & $\begin{array}{l}(0.027 \\
0.054 \\
0.081)\end{array}$ & $\begin{array}{c}(0.027, \\
0.054, \\
0.081)\end{array}$ \\
\hline$S 1$ & $\begin{array}{l}(0.000 \\
0.000 \\
0.027)\end{array}$ & $\begin{array}{c}(0.000, \\
0.000, \\
0.027)\end{array}$ & $\begin{array}{l}(0.000 \\
0.000 \\
0.027)\end{array}$ & $\begin{array}{l}(0.000 \\
0.000 \\
0.027)\end{array}$ & $\begin{array}{c}(0.000, \\
0.000, \\
0.000)\end{array}$ & $\begin{array}{l}(0.081 \\
0.108, \\
0.108)\end{array}$ & $\begin{array}{l}(0.054, \\
0.081, \\
0.108)\end{array}$ & $\begin{array}{l}(0.054, \\
0.081, \\
0.108)\end{array}$ & $\begin{array}{l}(0.000 \\
0.000 \\
0.027)\end{array}$ & $\begin{array}{c}(0.000 \\
0.000 \\
0.027)\end{array}$ & $\begin{array}{l}(0.000 \\
0.000 \\
0.027)\end{array}$ & $\begin{array}{c}(0.000, \\
0.000, \\
0.027)\end{array}$ \\
\hline S2 & $\begin{array}{l}(0.000 \\
0.027 \\
0.054)\end{array}$ & $\begin{array}{c}(0.000 \\
0.000 \\
0.027)\end{array}$ & $\begin{array}{l}(0.000 \\
0.000 \\
0.027)\end{array}$ & $\begin{array}{l}(0.000 \\
0.000, \\
0.027)\end{array}$ & $\begin{array}{c}(0.000 \\
0.027 \\
0.054)\end{array}$ & $\begin{array}{c}(0.000 \\
0.000 \\
0.000)\end{array}$ & $\begin{array}{l}(0.081 \\
0.108 \\
0.108)\end{array}$ & $\begin{array}{l}(0.054, \\
0.081, \\
0.108)\end{array}$ & $\begin{array}{l}(0.000 \\
0.000 \\
0.027)\end{array}$ & $\begin{array}{l}(0.000 \\
0.000, \\
0.027)\end{array}$ & $\begin{array}{l}(0.000 \\
0.000 \\
0.027)\end{array}$ & $\begin{array}{l}(0.000, \\
0.000, \\
0.027)\end{array}$ \\
\hline S3 & $\begin{array}{c}(0.027 \\
0.054 \\
0.081)\end{array}$ & $\begin{array}{l}(0.027, \\
0.054, \\
0.081)\end{array}$ & $\begin{array}{c}(0.054 \\
0.081 \\
0.108)\end{array}$ & $\begin{array}{l}(0.000 \\
0.000 \\
0.027)\end{array}$ & $\begin{array}{c}(0.054, \\
0.081, \\
0.108)\end{array}$ & $\begin{array}{c}(0.054, \\
0.081, \\
0.108)\end{array}$ & $\begin{array}{c}(0.000, \\
0.000, \\
0.000)\end{array}$ & $\begin{array}{c}(0.054, \\
0.081, \\
0.108)\end{array}$ & $\begin{array}{l}(0.054, \\
0.081, \\
0.108)\end{array}$ & $\begin{array}{c}(0.027 \\
0.054 \\
0.081)\end{array}$ & $\begin{array}{l}(0.054, \\
0.081, \\
0.108)\end{array}$ & $\begin{array}{c}(0.027, \\
0.054, \\
0.081)\end{array}$ \\
\hline S4 & $\begin{array}{l}(0.000 \\
0.027 \\
0.054)\end{array}$ & $\begin{array}{l}(0.000, \\
0.027 \\
0.054)\end{array}$ & $\begin{array}{l}(0.000 \\
0.000 \\
0.027)\end{array}$ & $\begin{array}{l}(0.000, \\
0.000, \\
0.027)\end{array}$ & $\begin{array}{l}(0.000 \\
0.027 \\
0.054)\end{array}$ & $\begin{array}{l}(0.000 \\
0.027 \\
0.054)\end{array}$ & $\begin{array}{l}(0.027, \\
0.054, \\
0.081)\end{array}$ & $\begin{array}{l}(0.000 \\
0.000 \\
0.000)\end{array}$ & $\begin{array}{l}(0.000 \\
0.000 \\
0.027)\end{array}$ & $\begin{array}{c}(0.000 \\
0.000, \\
0.027)\end{array}$ & $\begin{array}{l}(0.000 \\
0.000 \\
0.027)\end{array}$ & $\begin{array}{c}(0.000, \\
0.000, \\
0.027)\end{array}$ \\
\hline$G 1$ & $\begin{array}{l}(0.000 \\
0.027 \\
0.054)\end{array}$ & $\begin{array}{l}(0.000, \\
0.000, \\
0.027)\end{array}$ & $\begin{array}{l}(0.000 \\
0.000 \\
0.027)\end{array}$ & $\begin{array}{l}(0.000 \\
0.000, \\
0.027)\end{array}$ & $\begin{array}{l}(0.000 \\
0.027 \\
0.054)\end{array}$ & $\begin{array}{l}(0.054, \\
0.081, \\
0.108)\end{array}$ & $\begin{array}{c}(0.054, \\
0.081, \\
0.108)\end{array}$ & $\begin{array}{l}(0.000 \\
0.000 \\
0.027)\end{array}$ & $\begin{array}{l}(0.000, \\
0.000 \\
0.000)\end{array}$ & $\begin{array}{c}(0.000 \\
0.027 \\
0.054)\end{array}$ & $\begin{array}{l}(0.000 \\
0.027 \\
0.054)\end{array}$ & $\begin{array}{c}(0.000, \\
0.027, \\
0.054)\end{array}$ \\
\hline G2 & $\begin{array}{c}(0.027 \\
0.054 \\
0.081)\end{array}$ & $\begin{array}{l}(0.027, \\
0.054, \\
0.081)\end{array}$ & $\begin{array}{l}(0.000 \\
0.000 \\
0.027)\end{array}$ & $\begin{array}{l}(0.000 \\
0.027 \\
0.054)\end{array}$ & $\begin{array}{c}(0.000 \\
0.000, \\
0.027)\end{array}$ & $\begin{array}{l}(0.081 \\
0.108, \\
0.108)\end{array}$ & $\begin{array}{l}(0.081 \\
0.108, \\
0.108)\end{array}$ & $\begin{array}{l}(0.027, \\
0.054, \\
0.081)\end{array}$ & $\begin{array}{l}(0.054, \\
0.081, \\
0.108)\end{array}$ & $\begin{array}{c}(0.000 \\
0.000 \\
0.000)\end{array}$ & $\begin{array}{l}(0.027 \\
0.054, \\
0.081)\end{array}$ & $\begin{array}{c}(0.027 \\
0.054 \\
0.081)\end{array}$ \\
\hline G3 & $\begin{array}{c}(0.027 \\
0.054 \\
0.081)\end{array}$ & $\begin{array}{c}(0.027, \\
0.054, \\
0.081)\end{array}$ & $\begin{array}{l}(0.000 \\
0.000 \\
0.027)\end{array}$ & $\begin{array}{l}(0.000 \\
0.000 \\
0.027)\end{array}$ & $\begin{array}{l}(0.000 \\
0.000 \\
0.027)\end{array}$ & $\begin{array}{l}(0.081 \\
0.108 \\
0.108)\end{array}$ & $\begin{array}{l}(0.081 \\
0.108 \\
0.108)\end{array}$ & $\begin{array}{l}(0.027, \\
0.054, \\
0.081)\end{array}$ & $\begin{array}{l}(0.027 \\
0.054 \\
0.081)\end{array}$ & $\begin{array}{c}(0.000 \\
0.000 \\
0.027)\end{array}$ & $\begin{array}{l}(0.000 \\
0.000 \\
0.000)\end{array}$ & $\begin{array}{c}(0.000 \\
0.000 \\
0.027)\end{array}$ \\
\hline G4 & $\begin{array}{l}(0.000 \\
0.027 \\
0.054)\end{array}$ & $\begin{array}{l}(0.000, \\
0.000, \\
0.027)\end{array}$ & $\begin{array}{l}(0.000 \\
0.000 \\
0.027)\end{array}$ & $\begin{array}{l}(0.000 \\
0.000, \\
0.027)\end{array}$ & $\begin{array}{l}(0.000 \\
0.000, \\
0.027)\end{array}$ & $\begin{array}{l}(0.054, \\
0.081, \\
0.108)\end{array}$ & $\begin{array}{l}(0.054, \\
0.081, \\
0.108)\end{array}$ & $\begin{array}{l}(0.000, \\
0.027 \\
0.054)\end{array}$ & $\begin{array}{l}(0.000 \\
0.000 \\
0.027)\end{array}$ & $\begin{array}{l}(0.000 \\
0.000 \\
0.027)\end{array}$ & $\begin{array}{l}(0.000 \\
0.000 \\
0.027)\end{array}$ & $\begin{array}{c}(0.000 \\
0.000 \\
0.000)\end{array}$ \\
\hline
\end{tabular}

and play an important role in the implementation of sustainable methods and processes. To achieve the goals of sustainable development, it is necessary to pay close attention to environmental and social issues along with functional and economic aspects by architects, researchers, engineers, project managers, and other groups that are responsible for fundamental decisions at various stages of projects [78]. This study used a methodology similar to that proposed by Fernandez Sanchez and Rodriguez Lopez [79] to determine the sustainability criteria for selecting a project portfolio, and its methodology consists of three stages. First, the initial list of criteria is identified. Some of the identified criteria increasing the cost and time of the project are not feasible or may not be physically and technically feasible. As a result, it is necessary to filter the sustainability criteria before analyzing projects. Also, if a large number of criteria are considered, the cost of analysis and solution becomes very high and it becomes difficult to understand. If very few criteria are considered, important criteria may not be considered, and hence, important developments may not be achieved. To prioritize the identified criteria, opinions of experts and specialists in the field of projects are used. In the following, the steps taken to identify criteria based on this method are described:

Step 1: criteria identification. To identify the criteria, researches and studies related to the selection criteria of the project portfolio and project sustainability evaluation were examined and an initial list of sustainability criteria was extracted. This list includes 49 criteria as listed in Table 4.

Step 2: criteria classification. As mentioned earlier, Fernandez Sanchez and Rodriguez Lopez have proposed a structure for classifying the identified criteria in construction projects, called the sustainable breakdown structure, based on the three pillars of sustainability (economic, social, and environmental).

Step 3: criteria filtering. Experts' opinions and views are used to filter each of the identified criteria in order to remove those which are not important or incapable of implementation. 
TABLE 40: Total relationship matrix of sustainability criteria in fuzzy condition.

\begin{tabular}{|c|c|c|c|c|c|c|c|c|c|c|c|c|}
\hline & $E 1$ & $E 2$ & E3 & $E 4$ & $S 1$ & $S 2$ & S3 & S4 & G1 & G2 & G3 & G4 \\
\hline$E 1$ & $\begin{array}{l}(0.011, \\
0.039, \\
0.136)\end{array}$ & $\begin{array}{l}(0.086, \\
0.131, \\
0.217)\end{array}$ & $\begin{array}{l}(0.005, \\
0.014, \\
0.111)\end{array}$ & $\begin{array}{l}(0.000, \\
0.002, \\
0.089)\end{array}$ & $\begin{array}{l}(0.005, \\
0.052, \\
0.169)\end{array}$ & $\begin{array}{l}(0.011, \\
0.078, \\
0.220)\end{array}$ & $\begin{array}{l}(0.097, \\
0.170, \\
0.307)\end{array}$ & $\begin{array}{l}(0.037, \\
0.097 \\
0.244)\end{array}$ & $\begin{array}{l}(0.007, \\
0.054, \\
0.174)\end{array}$ & $\begin{array}{l}(0.032, \\
0.074, \\
0.183)\end{array}$ & $\begin{array}{c}(0.006, \\
0.051, \\
0.163)\end{array}$ & $\begin{array}{l}(0.033, \\
0.078, \\
0.191)\end{array}$ \\
\hline$E 2$ & $\begin{array}{l}(0.086, \\
0.136, \\
0.234)\end{array}$ & $\begin{array}{l}(0.011, \\
0.033, \\
0.120)\end{array}$ & $\begin{array}{l}(0.005, \\
0.014, \\
0.111)\end{array}$ & $\begin{array}{l}(0.000, \\
0.002, \\
0.089)\end{array}$ & $\begin{array}{l}(0.005, \\
0.052, \\
0.169)\end{array}$ & $\begin{array}{l}(0.011, \\
0.078, \\
0.220)\end{array}$ & $\begin{array}{l}(0.097, \\
0.170, \\
0.307)\end{array}$ & $\begin{array}{l}(0.037, \\
0.097 \\
0.244)\end{array}$ & $\begin{array}{l}(0.007, \\
0.054 \\
0.174)\end{array}$ & $\begin{array}{l}(0.032, \\
0.074, \\
0.183)\end{array}$ & $\begin{array}{l}(0.006, \\
0.051, \\
0.163)\end{array}$ & $\begin{array}{l}(0.033, \\
0.078, \\
0.191)\end{array}$ \\
\hline E3 & $\begin{array}{l}(0.003, \\
0.012, \\
0.111)\end{array}$ & $\begin{array}{l}(0.003, \\
0.011, \\
0.101)\end{array}$ & $\begin{array}{l}(0.005, \\
0.010, \\
0.057)\end{array}$ & $\begin{array}{l}(0.000, \\
0.000, \\
0.066)\end{array}$ & $\begin{array}{l}(0.005, \\
0.041, \\
0.130)\end{array}$ & $\begin{array}{l}(0.006, \\
0.021, \\
0.134)\end{array}$ & $\begin{array}{l}(0.084, \\
0.125, \\
0.231)\end{array}$ & $\begin{array}{l}(0.033, \\
0.072, \\
0.185)\end{array}$ & $\begin{array}{c}(0.005 \\
0.012 \\
0.105)\end{array}$ & $\begin{array}{l}(0.002, \\
0.008, \\
0.095)\end{array}$ & $\begin{array}{l}(0 . \\
0.0 \\
0.0\end{array}$ & $\begin{array}{l}(0.002, \\
0.009, \\
0.098)\end{array}$ \\
\hline$E 4$ & $\begin{array}{l}(0.032, \\
0.088, \\
0.232)\end{array}$ & $\begin{array}{l}(0.007 \\
0.057 \\
0.189)\end{array}$ & $\begin{array}{l}(0.004, \\
0.040, \\
0.150)\end{array}$ & $\begin{array}{l}(0.000, \\
0.003, \\
0.074)\end{array}$ & $\begin{array}{l}(0.031, \\
0.079 \\
0.213)\end{array}$ & $\begin{array}{l}(0.019, \\
0.062, \\
0.227)\end{array}$ & $\begin{array}{l}(0.074, \\
0.155, \\
0.343)\end{array}$ & $\begin{array}{l}(0.037, \\
0.100, \\
0.271)\end{array}$ & $\begin{array}{c}(0.062 \\
0.110 \\
0.246)\end{array}$ & $\begin{array}{l}(0.057, \\
0.100, \\
0.226)\end{array}$ & $\begin{array}{l}(0.033, \\
0.079, \\
0.207)\end{array}$ & $\begin{array}{l}(0.032, \\
0.079, \\
0.210)\end{array}$ \\
\hline$S 1$ & $\begin{array}{l}(0.002, \\
0.015, \\
0.123)\end{array}$ & $\begin{array}{l}(0.002, \\
0.011, \\
0.110)\end{array}$ & $\begin{array}{l}(0.003, \\
0.009 \\
0.090)\end{array}$ & $\begin{array}{l}(0.000, \\
0.000, \\
0.071)\end{array}$ & $\begin{array}{l}(0.003, \\
0.016, \\
0.089)\end{array}$ & $\begin{array}{l}(0.086, \\
0.126, \\
0.220)\end{array}$ & $\begin{array}{l}(0.064, \\
0.109, \\
0.249)\end{array}$ & $\begin{array}{l}(0.063, \\
0.104, \\
0.226)\end{array}$ & $\begin{array}{c}(0.004 \\
0.011 \\
0.114)\end{array}$ & $\begin{array}{l}(0.002, \\
0.008, \\
0.103)\end{array}$ & $\begin{array}{l}(0.004, \\
0.010, \\
0.107)\end{array}$ & $\begin{array}{l}(0.002, \\
0.008, \\
0.106)\end{array}$ \\
\hline$S 2$ & $\begin{array}{l}(0.003, \\
0.041, \\
0.144)\end{array}$ & $\begin{array}{l}(0.003, \\
0.016, \\
0.110)\end{array}$ & $\begin{array}{l}(0.005, \\
0.011, \\
0.088)\end{array}$ & $\begin{array}{l}(0.000, \\
0.000, \\
0.070)\end{array}$ & $\begin{array}{l}(0.005, \\
0.043, \\
0.138)\end{array}$ & $\begin{array}{l}(0.006, \\
0.024, \\
0.117)\end{array}$ & $\begin{array}{l}(0.085, \\
0.132, \\
0.244)\end{array}$ & $\begin{array}{l}(0.060, \\
0.102, \\
0.221)\end{array}$ & $\begin{array}{l}(0.005 \\
0.014 \\
0.112)\end{array}$ & $\begin{array}{l}02, \\
11, \\
02)\end{array}$ & $\begin{array}{l}(0.0 \\
0.0 \\
0.1\end{array}$ & $\begin{array}{l}(0.003, \\
0.011, \\
0.106)\end{array}$ \\
\hline S3 & & $\begin{array}{l}(0.033, \\
0.082, \\
0.221)\end{array}$ & $\begin{array}{l}(0.056, \\
0.089, \\
0.203)\end{array}$ & $\begin{array}{l}(0.000, \\
0.002, \\
0.106)\end{array}$ & $\begin{array}{l}(0.056, \\
0.106, \\
0.247)\end{array}$ & $\begin{array}{l}(0.072, \\
0.141, \\
0.312)\end{array}$ & $\begin{array}{l}(0.032, \\
0.092, \\
0.267)\end{array}$ & $\begin{array}{l}(0.068, \\
0.134, \\
0.314)\end{array}$ & $\begin{array}{l}(0.0 \\
0.1 \\
0.2\end{array}$ & $\begin{array}{l}(0.030, \\
0.071, \\
0.207)\end{array}$ & $\begin{array}{l}(0.0 \\
0.1 \\
0.2\end{array}$ & $\begin{array}{l}(0.030, \\
0.075, \\
0.215)\end{array}$ \\
\hline$S 4$ & $\begin{array}{l}(0.00 \\
0.03 \\
0.13\end{array}$ & $\begin{array}{l}(0.001, \\
0.037 \\
0.127)\end{array}$ & $\begin{array}{l}(0.002, \\
0.006, \\
0.082)\end{array}$ & $\begin{array}{l}(0.000, \\
0.000, \\
0.066)\end{array}$ & $\begin{array}{l}(0.002, \\
0.037, \\
0.129)\end{array}$ & $\begin{array}{l}(0.002, \\
0.043, \\
0.158)\end{array}$ & $\begin{array}{l}(0.028, \\
0.075 \\
0.209)\end{array}$ & $\begin{array}{l}(0.002, \\
0.018, \\
0.111)\end{array}$ & $\begin{array}{l}(0.002, \\
0.009, \\
0.105)\end{array}$ & $\begin{array}{l}(0.001, \\
0.008, \\
0.097)\end{array}$ & $\begin{array}{l}(0.002, \\
0.009, \\
0.098)\end{array}$ & $\begin{array}{l}(0.001, \\
0.009, \\
0.100)\end{array}$ \\
\hline$G 1$ & $\begin{array}{l}(0.002, \\
0.045, \\
0.160)\end{array}$ & $\begin{array}{l}(0.002, \\
0.016, \\
0.122)\end{array}$ & $\begin{array}{l}(0.003, \\
0.010, \\
0.097)\end{array}$ & $\begin{array}{l}(0.000, \\
0.001, \\
0.078)\end{array}$ & $\begin{array}{l}(0.003, \\
0.043, \\
0.149)\end{array}$ & $\begin{array}{l}(0.058, \\
0.111, \\
0.239)\end{array}$ & $\begin{array}{l}(0.060, \\
0.119, \\
0.270)\end{array}$ & $\begin{array}{l}(0.007, \\
0.031 \\
0.168)\end{array}$ & $\begin{array}{c}(0.003 \\
0.017 \\
0.101)\end{array}$ & $\begin{array}{l}(0.002, \\
0.037 \\
0.138)\end{array}$ & $\begin{array}{l}(0.003, \\
0.041, \\
0.143)\end{array}$ & $\begin{array}{l}(0.002, \\
0.039, \\
0.143)\end{array}$ \\
\hline G2 & $\begin{array}{l}(0.034, \\
0.092, \\
0.228)\end{array}$ & $\begin{array}{l}(0.034, \\
0.083, \\
0.207)\end{array}$ & $\begin{array}{l}(0.006, \\
0.016, \\
0.121)\end{array}$ & $\begin{array}{l}(0.000, \\
0.028, \\
0.121)\end{array}$ & $\begin{array}{l}(0.006, \\
0.032, \\
0.160)\end{array}$ & $\begin{array}{l}(0.095, \\
0.160, \\
0.289)\end{array}$ & $\begin{array}{l}(0.104, \\
0.183, \\
0.334)\end{array}$ & $\begin{array}{l}(0.041, \\
0.104, \\
0.264)\end{array}$ & $\begin{array}{c}(0.061 \\
0.109 \\
0.238)\end{array}$ & $\begin{array}{l}(0.005, \\
0.025, \\
0.122)\end{array}$ & $\begin{array}{l}(0.033, \\
0.079, \\
0.201)\end{array}$ & $\begin{array}{l}(0.032, \\
0.079, \\
0.204)\end{array}$ \\
\hline G3 & $\begin{array}{l}(0.033, \\
0.082, \\
0.200)\end{array}$ & $\begin{array}{l}(0.033, \\
0.076, \\
0.184)\end{array}$ & $\begin{array}{l}(0.005, \\
0.013, \\
0.105)\end{array}$ & $\begin{array}{l}(0.000, \\
0.001, \\
0.083)\end{array}$ & $\begin{array}{l}(0.005, \\
0.026, \\
0.137)\end{array}$ & $\begin{array}{l}(0.090, \\
0.143, \\
0.252)\end{array}$ & $\begin{array}{l}(0.098, \\
0.161, \\
0.290)\end{array}$ & $\begin{array}{l}(0.040, \\
0.093, \\
0.232)\end{array}$ & $\begin{array}{l}(0.033 \\
0.074 \\
0.187)\end{array}$ & $\begin{array}{l}(0.004, \\
0.019, \\
0.127)\end{array}$ & $\begin{array}{l}(0.005, \\
0.020, \\
0.102)\end{array}$ & $\begin{array}{l}(0.005, \\
0.020, \\
0.131)\end{array}$ \\
\hline G4 & $\begin{array}{l}(0.002, \\
0.040, \\
0.145)\end{array}$ & $\begin{array}{l}(0.002, \\
0.012, \\
0.109)\end{array}$ & $\begin{array}{l}(0.003, \\
0.009 \\
0.089)\end{array}$ & $\begin{array}{l}(0.000, \\
0.000, \\
0.070)\end{array}$ & $\begin{array}{l}(0.003, \\
0.015, \\
0.113)\end{array}$ & $\begin{array}{l}(0.058, \\
0.098 \\
0.218)\end{array}$ & $\begin{array}{l}(0.060, \\
0.106, \\
0.246)\end{array}$ & $\begin{array}{l}(0.007, \\
0.049, \\
0.174)\end{array}$ & $\begin{array}{l}(0.003, \\
0.011 \\
0.113)\end{array}$ & $\begin{array}{l}(0.002, \\
0.009, \\
0.102)\end{array}$ & $\begin{array}{l}(0.003, \\
0.011, \\
0.106)\end{array}$ & $\begin{array}{l}(0.002, \\
0.009, \\
0.080)\end{array}$ \\
\hline
\end{tabular}

TABLE 41: Importance weight of sustainability criteria in fuzzy condition.

\begin{tabular}{|c|c|c|c|c|c|c|c|}
\hline Criteria & $\widetilde{D}_{\mathbf{i}}+\widetilde{R}_{\mathbf{i}}$ & $\left(\widetilde{D}_{\mathbf{i}}+\widetilde{R}_{\mathbf{i}}\right)^{\mathbf{d e f}}$ & $\widetilde{D}_{\mathbf{i}}-\widetilde{R}_{\mathbf{i}}$ & $\left(\widetilde{D}_{\mathbf{i}}-\widetilde{R}_{\mathbf{i}}\right)^{\text {def }}$ & Influence & $\mathrm{W}_{\mathrm{i}}$ & $\mathbf{W}_{\mathbf{i}}^{\text {goal }}$ \\
\hline E1 & $(0.569,1.558,4.295)$ & 1.995 & $(-1.762,0.123,1.964)$ & 0.112 & Influence & 0.091 & 0.109 \\
\hline E2 & $(0.544,1.406,4.020)$ & 1.844 & $(-1.487,0.274,1.989)$ & 0.262 & Influence & 0.085 & 0.102 \\
\hline E3 & $(0.254,0.572,2.715)$ & 1.029 & $(-1.153,0.095,1.309)$ & 0.087 & Influence & 0.047 & 0.056 \\
\hline E4 & $(0.388,0.992,3.571)$ & 1.486 & $(-0.596,0.913,2.588)$ & 0.955 & Influence & 0.080 & 0.097 \\
\hline$S 1$ & $(0.363,0.969,3.449)$ & 1.438 & $(-1.607,-0.115,1.479)$ & -0.089 & Be-influence & 0.066 & 0.051 \\
\hline$S 2$ & $(0.694,1.504,4.164)$ & 1.966 & $(-2.425,-0.667,1.045)$ & -0.679 & B-influence & 0.095 & 0.074 \\
\hline$S 3$ & $(1.411,2.684,6.116)$ & 3.224 & $(-2.771,-0.511,1.934)$ & -0.464 & Be-influence & 0.148 & 0.115 \\
\hline$S 4$ & $(0.472,1.293,4.075)$ & 1.783 & $(-2.615,-0.714,0.988)$ & -0.764 & Be-influence & 0.088 & 0.069 \\
\hline$G 1$ & $(0.397,1.091,3.729)$ & 1.577 & $(-1.774,-0.071,1.558)$ & -0.089 & Be-influence & 0.072 & 0.078 \\
\hline G2 & $(0.620,1.435,4.176)$ & 1.916 & $(-1.235,0.545,2.321)$ & 0.544 & Influence & 0.091 & 0.098 \\
\hline G3 & $(0.511,1.205,3.760)$ & 1.670 & $(-1.380,0.253,1.869)$ & 0.249 & Influence & 0.077 & 0.083 \\
\hline G4 & $(0.322,0.864,3.338)$ & 1.347 & $(-1.627,-0.126,1.389)$ & -0.123 & Be-influence & 0.062 & 0.067 \\
\hline
\end{tabular}

3.2.Z-Number DEMATEL Approach for Measuring Weights of Criteria and Their Impacts. This technique is based on diagrams (oriented graphs) that use the judgment of experts to identify the factors in a system and, by applying the principles of graph theory, to extract the effective or influential relationships of elements (causal and effect, reciprocal) and provides structural with systematic and orderly sequence, and ultimately, the weight of the criteria can be obtained. Companies can improve the effectiveness of specific criteria based on the diagram map (directional graph). The DEMATEL method converts the relationship between causal and effect criteria into a smart structural model of the system. This section examines the effectiveness or influence of filtered sustainability criteria and also 
TABLE 42: Decision matrix in fuzzy condition.

\begin{tabular}{|c|c|c|c|c|c|c|c|c|c|c|c|c|}
\hline & $E 1$ & $E 2$ & E3 & $E 4$ & $S 1$ & $S 2$ & $S 3$ & S4 & G1 & G2 & G3 & G4 \\
\hline$P 1$ & $\begin{array}{c}(1.235,1.3 \\
1.365)\end{array}$ & $\begin{array}{c}(19.627, \\
20.66, \\
21.693)\end{array}$ & $\begin{array}{c}(26, \\
29,33)\end{array}$ & $\begin{array}{c}(0.5, \\
0.75,1)\end{array}$ & $\begin{array}{l}(0,0 \\
0.25)\end{array}$ & $\begin{array}{c}(0.25 \\
0.5,0.75)\end{array}$ & $\begin{array}{c}(0.75,1 \\
1)\end{array}$ & $\begin{array}{l}(0,0, \\
0.25)\end{array}$ & $\begin{array}{l}(0,0 \\
0.25)\end{array}$ & $\begin{array}{c}(0.5, \\
0.75,1)\end{array}$ & $\begin{array}{c}(0.5, \\
0.75,1)\end{array}$ & $\begin{array}{c}(0.75,1, \\
1)\end{array}$ \\
\hline$P 2$ & $\begin{array}{c}(1.615,1.7 \\
1.785)\end{array}$ & $\begin{array}{c}(23.902, \\
25.16 \\
26.418)\end{array}$ & $\begin{array}{c}(32, \\
35,39)\end{array}$ & $\begin{array}{c}(0.5 \\
0.75,1)\end{array}$ & $\begin{array}{c}(0,0.25 \\
0.5)\end{array}$ & $\begin{array}{l}(0,0 \\
0.25)\end{array}$ & $\begin{array}{c}(0.5, \\
0.75,1)\end{array}$ & $\begin{array}{c}(0.25 \\
0.5,0.75)\end{array}$ & $\begin{array}{c}(0.75,1, \\
1)\end{array}$ & $\begin{array}{c}(0.75,1, \\
1)\end{array}$ & $\begin{array}{c}(0.5 \\
0.75,1)\end{array}$ & $\begin{array}{l}(0,0 \\
0.25)\end{array}$ \\
\hline$P 3$ & $\begin{array}{c}(0.827, \\
0.87,0.914)\end{array}$ & $\begin{array}{r}(14.44,1 \\
15.96\end{array}$ & $\begin{array}{c}(14, \\
17,21)\end{array}$ & $\begin{array}{l}(0,0 \\
0.25)\end{array}$ & $\begin{array}{l}(0,0 \\
0.25)\end{array}$ & $\begin{array}{c}(0,0.25 \\
0.5)\end{array}$ & $\begin{array}{c}(0.75,1, \\
1)\end{array}$ & $\begin{array}{c}(0.75,1, \\
1)\end{array}$ & $\begin{array}{c}(0.75,1 \\
1)\end{array}$ & $\begin{array}{c}(0.25, \\
0.5,0.75)\end{array}$ & $\begin{array}{l}(0,0 \\
0.25)\end{array}$ & $\begin{array}{c}(0.25, \\
0.5,0.75)\end{array}$ \\
\hline$P 4$ & $\begin{array}{c}(1.881 \\
1.98,2.079)\end{array}$ & $\begin{array}{r}(25.365 \\
28.03\end{array}$ & $\begin{array}{c}(43, \\
46,50)\end{array}$ & $\begin{array}{c}(0.5, \\
0.75,1)\end{array}$ & $\begin{array}{c}(0,0.25 \\
0.5)\end{array}$ & $\begin{array}{l}(0,0 \\
0.25)\end{array}$ & $\begin{array}{c}(0.75,1 \\
1)\end{array}$ & $\begin{array}{c}(0,0.25 \\
0.5)\end{array}$ & $\begin{array}{c}(0.25 \\
0.5,0.75)\end{array}$ & $\begin{array}{c}(0,0.25 \\
0.5)\end{array}$ & $\begin{array}{c}(0.75,1 \\
1)\end{array}$ & $\begin{array}{c}(0.5 \\
0.75,1)\end{array}$ \\
\hline P5 & $\begin{array}{c}(1.064, \\
1.12,1.176)\end{array}$ & $\begin{array}{c}(18.459, \\
19.43 \\
20.402)\end{array}$ & $\begin{array}{c}(21, \\
24,28)\end{array}$ & $\begin{array}{l}(0,0, \\
0.25)\end{array}$ & $\begin{array}{l}(0,0 \\
0.25)\end{array}$ & $\begin{array}{c}(0,0.25 \\
0.5)\end{array}$ & $\begin{array}{c}(0,0.25 \\
0.5)\end{array}$ & $\begin{array}{l}(0,0 \\
0.25)\end{array}$ & $\begin{array}{l}(0,0 \\
0.25)\end{array}$ & $\begin{array}{c}(0.25, \\
0.5,0.75)\end{array}$ & $\begin{array}{l}(0,0 \\
0.25)\end{array}$ & $\begin{array}{l}(0,0 \\
0.25)\end{array}$ \\
\hline P6 & $\begin{array}{c}(2.014 \\
2.12,2.226)\end{array}$ & $\begin{array}{c}(28.595,30.1 \\
31.605)\end{array}$ & $\begin{array}{c}(48, \\
51,55)\end{array}$ & $\begin{array}{c}(0.5 \\
0.75,1)\end{array}$ & $\begin{array}{c}(0.25, \\
0.5,0.75)\end{array}$ & $\begin{array}{c}(0.25 \\
0.5,0.75)\end{array}$ & $\begin{array}{c}(0.25 \\
0.5,0.75)\end{array}$ & $\begin{array}{c}(0.25 \\
0.5,0.75)\end{array}$ & $\begin{array}{c}(0.5 \\
0.75,1)\end{array}$ & & $\begin{array}{c}(0,0.25 \\
0.5)\end{array}$ & $\begin{array}{c}(0.25, \\
0.5,0.75)\end{array}$ \\
\hline$P 7$ & $\begin{array}{c}(1.777 \\
1.87,1.964)\end{array}$ & $\begin{array}{c}(22.582, \\
23.77 \\
24.959)\end{array}$ & $\begin{array}{c}(39, \\
42,46)\end{array}$ & $\begin{array}{l}(0,0, \\
0.25)\end{array}$ & $\begin{array}{c}(0,0.25 \\
0.5)\end{array}$ & $\begin{array}{l}(0,0, \\
0.25)\end{array}$ & $\begin{array}{c}(0,0.25 \\
0.5)\end{array}$ & $\begin{array}{c}(0.75,1, \\
1)\end{array}$ & $\begin{array}{l}(0,0, \\
0.25)\end{array}$ & $\begin{array}{c}(0.25, \\
0.5,0.75)\end{array}$ & $\begin{array}{c}(0.5 \\
0.75,1)\end{array}$ & $\begin{array}{c}(0.5 \\
0.75,1)\end{array}$ \\
\hline$P 8$ & $\begin{array}{c}(0.931, \\
0.98,1.029)\end{array}$ & $\begin{array}{c}(16.084, \\
16.93, \\
17.777)\end{array}$ & $\begin{array}{c}(16, \\
19,23)\end{array}$ & $\begin{array}{c}(0.5 \\
0.75,1)\end{array}$ & $\begin{array}{c}(0.75,1 \\
1)\end{array}$ & $\begin{array}{c}(0.75,1 \\
1)\end{array}$ & $\begin{array}{c}(0.75,1 \\
1)\end{array}$ & $\begin{array}{c}(0.5 \\
0.75,1)\end{array}$ & $\begin{array}{c}(0,0.25 \\
0.5)\end{array}$ & $\begin{array}{c}(0.5 \\
0.75,1)\end{array}$ & $\begin{array}{l}(0,0, \\
0.25)\end{array}$ & $\begin{array}{c}(0.5, \\
0.75,1)\end{array}$ \\
\hline
\end{tabular}

TABLe 43: Normalized decision matrix in fuzzy condition.

\begin{tabular}{|c|c|c|c|c|c|c|c|c|c|c|c|c|}
\hline Weight & $\begin{array}{c}0.109 \\
E 1\end{array}$ & $\begin{array}{c}0.102 \\
E 2\end{array}$ & $\begin{array}{c}0.056 \\
E 3\end{array}$ & $\begin{array}{c}0.097 \\
E 4\end{array}$ & $\begin{array}{c}0.051 \\
S 1\end{array}$ & $\begin{array}{c}0.074 \\
S 2\end{array}$ & $\begin{array}{c}0.115 \\
S 3\end{array}$ & $\begin{array}{c}0.069 \\
S 4\end{array}$ & $\begin{array}{c}0.078 \\
G 1\end{array}$ & $\begin{array}{c}0.098 \\
G 2\end{array}$ & $\begin{array}{c}0.083 \\
G 3\end{array}$ & $\begin{array}{c}0.067 \\
G 4\end{array}$ \\
\hline$P 1$ & $\begin{array}{l}(0.464, \\
0.586, \\
0.708)\end{array}$ & $\begin{array}{c}(0.402, \\
0.55, \\
0.698)\end{array}$ & $\begin{array}{l}(0.122, \\
0.293, \\
0.463)\end{array}$ & $\begin{array}{l}(-0.5 \\
0,0.5)\end{array}$ & $\begin{array}{c}(0.5,1, \\
1)\end{array}$ & $\begin{array}{c}(0,0.5 \\
0.75)\end{array}$ & $\begin{array}{l}(-0.25 \\
0,0.25)\end{array}$ & $\begin{array}{c}(0.5,1, \\
1)\end{array}$ & $\begin{array}{l}(-0.25 \\
0,0.25)\end{array}$ & $\begin{array}{c}-0.25, \\
0.25, \\
0.5)\end{array}$ & $\begin{array}{c}(0.25 \\
0.75,1)\end{array}$ & $\begin{array}{l}(-0.25 \text {, } \\
0,0.25)\end{array}$ \\
\hline$P 2$ & $\begin{array}{c}(0.164, \\
0.3,0.437)\end{array}$ & $\begin{array}{c}(0.127 \\
0.288 \\
0.449)\end{array}$ & $\begin{array}{c}(0.268 \\
0.439 \\
0.61)\end{array}$ & $\begin{array}{l}(-0.5 \\
0,0.5)\end{array}$ & $\begin{array}{c}(0.25, \\
0.75,1)\end{array}$ & $\begin{array}{c}(0.5,1 \\
1)\end{array}$ & $\begin{array}{c}(-0.25 \\
0.25 \\
0.5)\end{array}$ & $\begin{array}{c}(0,0.5 \\
0.75)\end{array}$ & $\begin{array}{c}(0.5,1 \\
1)\end{array}$ & $\begin{array}{l}(-0.25 \\
0,0.25)\end{array}$ & $\begin{array}{c}(0.25 \\
0.75,1)\end{array}$ & $\begin{array}{c}(0.5,1, \\
1)\end{array}$ \\
\hline$P 3$ & $\begin{array}{c}(0.786, \\
0.893,1)\end{array}$ & $\begin{array}{c}(0.736 \\
0.868,1)\end{array}$ & $\begin{array}{c}(-0.171,0 \\
0.171)\end{array}$ & $\begin{array}{c}(0.25, \\
0.75,1)\end{array}$ & $\begin{array}{c}(0.5,1, \\
1)\end{array}$ & $\begin{array}{c}(0.25 \\
0.75,1)\end{array}$ & $\begin{array}{l}(-0.25 \\
0,0.25)\end{array}$ & $\begin{array}{l}(-0.25 \\
0,0.25)\end{array}$ & $\begin{array}{c}(0.5,1 \\
1)\end{array}$ & $\begin{array}{c}(0,0.5, \\
0.75)\end{array}$ & $\begin{array}{l}(-0.25 \\
0,0.25)\end{array}$ & $\begin{array}{c}(0,0.5 \text {, } \\
0.75)\end{array}$ \\
\hline$P 4$ & $\begin{array}{c}(-0.046 \\
0.1,0.247)\end{array}$ & $\begin{array}{c}(0.033 \\
0.198 \\
0.364)\end{array}$ & $\begin{array}{l}(0.537 \\
0.707 \\
0.878)\end{array}$ & $\begin{array}{l}(-0.5 \\
0,0.5)\end{array}$ & $\begin{array}{c}(0.25, \\
0.75,1)\end{array}$ & $\begin{array}{c}(0.5,1 \\
1)\end{array}$ & $\begin{array}{l}(-0.25 \\
0,0.25)\end{array}$ & $\begin{array}{c}(0.25 \\
0.75,1)\end{array}$ & $\begin{array}{c}(0,0.5 \\
0.75)\end{array}$ & $\begin{array}{c}(0.25 \\
0.75,1)\end{array}$ & $\begin{array}{c}(0.5,1, \\
1)\end{array}$ & $\begin{array}{c}(-0.25, \\
0.25, \\
0.5)\end{array}$ \\
\hline P5 & $\begin{array}{l}(0.599, \\
0.715, \\
0.831)\end{array}$ & $\begin{array}{l}(0.477, \\
0.622, \\
0.766)\end{array}$ & $\begin{array}{c}(0,0.171 \\
0.341)\end{array}$ & $\begin{array}{c}(0.25 \\
0.75,1)\end{array}$ & $\begin{array}{c}(0.5,1, \\
1)\end{array}$ & $\begin{array}{c}(0.25 \\
0.75,1)\end{array}$ & $\begin{array}{c}(0.25 \\
0.75,1)\end{array}$ & $\begin{array}{c}(0.5,1 \\
1)\end{array}$ & $\begin{array}{c}(-0.25 \\
0,0.25)\end{array}$ & $\begin{array}{c}(0,0.5 \\
0.75)\end{array}$ & $\begin{array}{c}(-0.25 \\
0,0.25)\end{array}$ & $\begin{array}{c}(0.5,1, \\
1)\end{array}$ \\
\hline P6 & $\begin{array}{c}(-0.152,0 \\
0.152)\end{array}$ & $\begin{array}{l}(-0.175 \\
0,0.175)\end{array}$ & $\begin{array}{c}(0.659, \\
0.829,1)\end{array}$ & $\begin{array}{l}(-0.5 \\
0,0.5)\end{array}$ & $\begin{array}{c}(0,0.5 \\
0.75)\end{array}$ & $\begin{array}{c}(0,0.5 \\
0.75)\end{array}$ & $\begin{array}{c}(0,0.5 \\
0.75)\end{array}$ & $\begin{array}{c}(0,0.5 \\
0.75)\end{array}$ & $\begin{array}{c}(0.25 \\
0.75,1)\end{array}$ & $\begin{array}{c}(0.5,1, \\
1)\end{array}$ & $\begin{array}{c}(-0.25 \\
0.25 \\
0.5)\end{array}$ & $\begin{array}{c}(0,0.5, \\
0.75)\end{array}$ \\
\hline P7 & $\begin{array}{l}(0.036, \\
0.179 \\
0.321)\end{array}$ & $\begin{array}{l}(0.212, \\
0.369, \\
0.526)\end{array}$ & $\begin{array}{c}(0.439 \\
0.61,0.78)\end{array}$ & $\begin{array}{c}(0.25 \\
0.75,1)\end{array}$ & $\begin{array}{c}(0.25, \\
0.75,1)\end{array}$ & $\begin{array}{c}(0.5,1, \\
1)\end{array}$ & $\begin{array}{c}(0.25 \\
0.75,1)\end{array}$ & $\begin{array}{l}(-0.25 \\
0,0.25)\end{array}$ & $\begin{array}{l}(-0.25 \\
0,0.25)\end{array}$ & $\begin{array}{c}(0,0.5 \\
0.75)\end{array}$ & $\begin{array}{c}(0.25, \\
0.75,1)\end{array}$ & $\begin{array}{c}(-0.25 \\
0.25 \\
0.5)\end{array}$ \\
\hline P8 & $\begin{array}{l}(0.704, \\
0.815 \\
0.926)\end{array}$ & $\begin{array}{l}(0.63, \\
0.767, \\
0.904)\end{array}$ & $\begin{array}{c}(-0.122 \\
0.049 \\
0.22)\end{array}$ & $\begin{array}{l}(-0.5 \\
0,0.5)\end{array}$ & $\begin{array}{l}(-0.25 \\
0,0.25)\end{array}$ & $\begin{array}{l}(-0.25 \\
0,0.25)\end{array}$ & $\begin{array}{c}(-0.25 \\
0,0.25)\end{array}$ & $\begin{array}{c}(-0.25 \\
0.25 \\
0.5)\end{array}$ & $\begin{array}{c}(-0.25 \\
0.25 \\
0.5)\end{array}$ & $\begin{array}{c}(-0.25 \\
0.25 \\
0.5)\end{array}$ & $\begin{array}{l}(-0.25 \\
0,0.25)\end{array}$ & $\begin{array}{c}(-0.25 \\
0.25 \\
0.5)\end{array}$ \\
\hline
\end{tabular}

determines the importance of sustainability criteria by using $\mathrm{Z}$-number to rank projects. Lane and $\mathrm{Wu}$ [80] provided the following steps for performing the fuzzy DEMATEL method:

Step 1: form a group of experts to gather their knowledge to solve the problem.
Step 2: determine the criteria being evaluated as well as design the linguistic scales in fuzzy conditions and define a new linguistic variable for the Z-number.

In this step, the factors and criteria of the study are identified using the opinions of experts. The criteria to be evaluated will be selected according to the areas 
TABLE 44: Project ranking in fuzzy condition.

\begin{tabular}{|c|c|c|c|c|c|c|c|c|c|c|}
\hline \multirow{2}{*}{ Project } & \multirow{2}{*}{$S$} & \multirow{2}{*}{$S g$} & \multirow{2}{*}{$R$} & \multirow{2}{*}{$R g$} & \multirow{2}{*}{$Q$} & \multirow{2}{*}{$\mathrm{Qg}$} & \multicolumn{3}{|c|}{ Rank by } & \multirow{2}{*}{ Rank } \\
\hline & & & & & & & $\mathrm{R}$ & $S$ & Q & \\
\hline$P 1$ & $(0.049,0.389,0.598)$ & 0.356 & $(0.049,0.076,0.082)$ & 0.071 & $(-0.471,0.085,0.676)$ & 0.094 & 1 & 2 & 1 & 1 \\
\hline$P 2$ & $(0.1,0.486,0.675)$ & 0.437 & $(0.041,0.081,0.082)$ & 0.071 & $(-0.495,0.175,0.72)$ & 0.144 & 2 & 5 & 3 & 3 \\
\hline$P 3$ & $(0.185,0.52,0.704)$ & 0.483 & $(0.083,0.094,0.105)$ & 0.094 & $(-0.171,0.278,0.887)$ & 0.318 & 8 & 7 & 7 & 7 \\
\hline$P 4$ & $(0.077,0.458,0.666)$ & 0.415 & $(0.041,0.082,0.093)$ & 0.075 & $(-0.505,0.165,0.787)$ & 0.153 & 3 & 4 & 4 & 4 \\
\hline P5 & $(0.24,0.605,0.775)$ & 0.556 & $(0.063,0.087,0.116)$ & 0.088 & $(-0.267,0.284,1)$ & 0.325 & 7 & 8 & 8 & 8 \\
\hline P6 & $(0.009,0.421,0.647)$ & 0.375 & $(0.047,0.093,0.093)$ & 0.081 & $(-0.509,0.215,0.776)$ & 0.174 & 4 & 3 & 5 & 5 \\
\hline P7 & $(0.117,0.487,0.692)$ & 0.446 & $(0.039,0.087,0.116)$ & 0.082 & $(-0.494,0.215,0.952)$ & 0.222 & 5 & 6 & 6 & 6 \\
\hline P8 & $(-0.078,0.243,0.484)$ & 0.223 & $(0.074,0.086,0.097)$ & 0.086 & $(-0.382,0.062,0.707)$ & 0.112 & 6 & 1 & 2 & 2 \\
\hline
\end{tabular}

TABLe 45: Project ranking table (8 projects).

\begin{tabular}{|c|c|c|c|c|c|c|c|c|c|c|}
\hline Project & $S$ & $\widetilde{S}_{\text {def }}^{Z}$ & $R$ & $\widetilde{R}_{\mathrm{def}}^{Z}$ & $Q$ & $\widetilde{Q}_{\text {def }}^{Z}$ & $R$ & $S$ & Q & Rank \\
\hline$P 1$ & $(0.036,0.328,0.574)$ & 0.316 & $(0.051,0.064,0.081)$ & 0.065 & $(-0.517,0.035,0.708)$ & 0.065 & 2 & 2 & 1 & 1 \\
\hline$P 2$ & $(0.074,0.423,0.657)$ & 0.394 & $(0.029,0.065,0.087)$ & 0.062 & $(-0.626,0.102,0.795)$ & 0.093 & 1 & 4 & 2 & 2 \\
\hline$P 3$ & $(0.204,0.511,0.74)$ & 0.492 & $(0.086,0.097,0.109)$ & 0.097 & $(-0.19,0.362,0.986)$ & 0.38 & 8 & 7 & 8 & $\boldsymbol{x}$ \\
\hline P4 & $(0.098,0.434,0.656)$ & 0.406 & $(0.033,0.071,0.106)$ & 0.07 & $(-0.589,0.144,0.914)$ & 0.154 & 3 & 6 & 3 & $\boldsymbol{x}$ \\
\hline P5 & $(0.202,0.534,0.762)$ & 0.508 & $(0.065,0.083,0.109)$ & 0.085 & $(-0.319,0.285,1)$ & 0.313 & 6 & 8 & 7 & $\mathbf{x}$ \\
\hline P6 & $(0.002,0.364,0.624)$ & 0.339 & $(0.052,0.086,0.106)$ & 0.083 & $(-0.527,0.197,0.893)$ & 0.19 & 5 & 3 & 5 & $\boldsymbol{x}$ \\
\hline$P 7$ & $(0.101,0.426,0.652)$ & 0.401 & $(0.033,0.085,0.109)$ & 0.078 & $(-0.588,0.23,0.93)$ & 0.201 & 4 & 5 & 6 & $\mathbf{x}$ \\
\hline$P 8$ & $(-0.024,0.273,0.545)$ & 0.266 & $(0.077,0.089,0.101)$ & 0.089 & $(-0.391,0.157,0.811)$ & 0.183 & 7 & 1 & 4 & $x$ \\
\hline
\end{tabular}

TABLE 46: Probabilistic linguistic scoring matrix of sustainability criteria for proposed projects.

\begin{tabular}{|c|c|c|c|c|c|c|c|c|c|c|c|c|}
\hline & $E 1$ & $E 2$ & E3 & $E 4$ & $S 1$ & $S 2$ & S3 & $S 4$ & G1 & G2 & G3 & G4 \\
\hline$P 1$ & M-ML & B-ML & M-L & M-L & VB-L & M-L & B-C & B-C & VB-C & B-ML & VB-C & M-ML \\
\hline$P 2$ & S-L & B-M & S-L & VS-M & S-M & M-ML & B-L & $M-W$ & VS-L & B-ML & VS-L & VS-M \\
\hline$P 3$ & VB-M & S-FI & B-W & VB-M & B-W & B-M & S-L & S-W & S-M & VB-FI & S-M & B-L \\
\hline$P 4$ & B-ML & S-L & B-L & M-L & VS-C & B-C & M-ML & $\mathrm{M}-\mathrm{C}$ & S-L & B-L & M-ML & B-ML \\
\hline P5 & M-ML & M-FI & VS-FI & M-M & S-FI & VS-ML & B-W & B-C & S-M & M-ML & S-M & VS-L \\
\hline$P 6$ & VB-W & VB-M & B-L & VS-ML & M-W & B-M & S-W & B-ML & B-W & S-W & B-ML & M-M \\
\hline$P 7$ & VS-M & M-M & B-C & M-ML & M-L & S-M & S-M & B-C & VS-FI & M-FI & S-M & S-W \\
\hline$P 8$ & B-L & B-ML & VB-C & VB-L & B-C & B-ML & B-ML & VB-ML & B-ML & B-ML & B-ML & B-L \\
\hline$P 9$ & $\mathrm{M}-\mathrm{W}$ & M-L & B-M & B-ML & M-L & B-FI & B-M & B-FI & B-ML & VB-L & VB-FI & B-FI \\
\hline$P 10$ & M-ML & VS-C & M-C & M-L & S-L & M-L & $\mathrm{M}-\mathrm{C}$ & S-ML & M-C & S-L & VS-ML & VS-ML \\
\hline$P 11$ & VB-ML & $\mathrm{S}-\mathrm{C}$ & B-L & S-ML & VB-W & S-W & M-ML & S-L & B-M & B-M & M-W & S-C \\
\hline$P 12$ & M-W & S-L & M-L & M-L & B-M & S-L & B-L & M-L & S-L & S-L & B-L & M-M \\
\hline$P 13$ & VS-L & VS-W & B-M & S-ML & B-L & VS-M & S-ML & M-L & VS-L & B-M & S-M & B-W \\
\hline$P 14$ & M-L & $\mathrm{M}-\mathrm{W}$ & B-C & M-L & B-L & S-ML & VS-C & S-C & VS-W & M-L & B-M & M-M \\
\hline$P 15$ & M-ML & VS-C & M-C & VS-L & S-ML & B-C & S-L & VB-ML & B-L & S-L & S-L & VB-L \\
\hline$P 16$ & B-L & M-ML & B-ML & M-M & B-FI & M-W & VB-M & M-ML & $\mathrm{M}-\mathrm{C}$ & VB-W & M-M & M-W \\
\hline$P 17$ & S-M & B-M & S-W & M-W & B-ML & M-ML & M-M & S-ML & B-ML & S-M & M-W & S-W \\
\hline$P 18$ & M-C & B-ML & VB-ML & B-W & B-FI & $\mathrm{M}-\mathrm{C}$ & $\mathrm{S}-\mathrm{C}$ & M-W & S-M & B-L & M-FI & B-W \\
\hline$P 19$ & M-M & VS-W & VS-W & B-ML & S-C & $M-W$ & B-M & S-M & B-ML & VS-L & M-ML & S-ML \\
\hline$P 20$ & VB-C & VS-C & B-L & M-L & B-L & VB-C & M-L & B-ML & VS-ML & S-ML & M-L & M-C \\
\hline
\end{tabular}

studied. The linguistic scales used in this method and their corresponding values are given in Table 5. The fuzzy numbers used in this study are triangular fuzzy. As can be seen, this variable is the same as the DEMATEL variable, except that fuzzy numbers have been used.

In Z-number, the word variable is defined as follows. In Table 6, the used linguistic expression and its corresponding fuzzy numbers of Z-number are stated in the probabilistic condition.
To calculate the linguistic variable of Z-number, we use equation (14) and an example of its calculation is as follows:

$$
\begin{aligned}
& \text { high influence - fairly impossible } \\
& \begin{aligned}
A^{\prime} & =[\sqrt{\alpha} d, \sqrt{\alpha} e, \sqrt{\alpha} f]=\left[d^{\prime}, e^{\prime}, f^{\prime}\right] \\
& =0.63 \times[0.50,0.75,1.00]=[0.32,0.47,0.63] .
\end{aligned}
\end{aligned}
$$

In Table 6, the linguistic variable of $\mathrm{Z}$-number is prepared. As can be seen, this variable is similar to the 
TABLE 47: Quantified probabilistic linguistic scoring spectrum for sustainability criteria for proposed projects.

\begin{tabular}{|c|c|c|c|c|c|c|c|c|c|c|c|c|}
\hline & $E 1$ & $E 2$ & E3 & $E 4$ & $S 1$ & $S 2$ & S3 & S4 & G1 & G2 & G3 & G4 \\
\hline$P 1$ & $\begin{array}{l}0.238 \\
0.475, \\
0.713)\end{array}$ & $\begin{array}{c}(0.475 \\
0.713 \\
0.95)\end{array}$ & $\begin{array}{l}(0.223, \\
0.445, \\
0.668)\end{array}$ & $\begin{array}{l}(0.223, \\
0.445, \\
0.668)\end{array}$ & $\begin{array}{c}0.668 \\
0.89 \\
0.89)\end{array}$ & $\begin{array}{l}0.223, \\
0.445, \\
0.668)\end{array}$ & $\begin{array}{c}(0.5 \\
0.75,1)\end{array}$ & $\begin{array}{c}(0.5 \\
0.75,1)\end{array}$ & $\begin{array}{c}(0.75,1 \\
1)\end{array}$ & $\begin{array}{c}(0.475 \\
0.713 \\
0.95)\end{array}$ & $\begin{array}{c}(0.75,1 \\
1)\end{array}$ & $\begin{array}{c}(0.238 \\
0.475 \\
0.713)\end{array}$ \\
\hline$P 2$ & $\begin{array}{c}(0,0.223 \\
0.445)\end{array}$ & $\begin{array}{c}(0.385 \\
0.578 \\
0.77)\end{array}$ & $\begin{array}{c}(0,0.223 \\
0.445)\end{array}$ & $\begin{array}{l}(0,0 \\
0.193)\end{array}$ & $\begin{array}{c}(0,0.193 \\
0.385)\end{array}$ & $\begin{array}{l}(0.238, \\
0.475, \\
0.713)\end{array}$ & $\begin{array}{c}(0.445 \\
0.668 \\
0.89)\end{array}$ & $\begin{array}{l}(0.178, \\
0.355 \\
0.533)\end{array}$ & $\begin{array}{c}(0,0, \\
0.223)\end{array}$ & $\begin{array}{c}(0.475 \\
0.713 \\
0.95)\end{array}$ & $\begin{array}{c}(0,0, \\
0.223)\end{array}$ & $\begin{array}{c}(0,0, \\
0.193)\end{array}$ \\
\hline$P 3$ & $\begin{array}{c}(0.578 \\
0.77 \\
0.77)\end{array}$ & $\begin{array}{c}(0,0.158 \\
0.315)\end{array}$ & $\begin{array}{c}(0.355 \\
0.533 \\
0.71)\end{array}$ & $\begin{array}{c}(0.578 \\
0.77 \\
0.77)\end{array}$ & $\begin{array}{c}(0.355 \\
0.533 \\
0.71)\end{array}$ & $\begin{array}{c}(0.385 \\
0.578 \\
0.77)\end{array}$ & $\begin{array}{c}(0,0.223 \\
0.445)\end{array}$ & $\begin{array}{c}(0,0.178 \\
0.355)\end{array}$ & $\begin{array}{c}(0,0.193 \\
0.385)\end{array}$ & $\begin{array}{c}(0.473 \\
0.63 \\
0.63)\end{array}$ & $\begin{array}{c}(0,0.193 \\
0.385)\end{array}$ & $\begin{array}{c}(0.445 \\
0.668 \\
0.89)\end{array}$ \\
\hline$P 4$ & $\begin{array}{c}(0.475 \\
0.713 \\
0.95)\end{array}$ & $\begin{array}{c}(0,0.223 \\
0.445)\end{array}$ & $\begin{array}{c}(0.445 \\
0.668 \\
0.89)\end{array}$ & $\begin{array}{l}(0.223, \\
0.445, \\
0.668)\end{array}$ & $\begin{array}{l}(0,0, \\
0.25)\end{array}$ & $\begin{array}{c}(0.5, \\
0.75,1)\end{array}$ & $\begin{array}{l}(0.238 \\
0.475 \\
0.713)\end{array}$ & $\begin{array}{c}(0.25 \\
0.5,0.75)\end{array}$ & $\begin{array}{c}(0,0.223 \\
0.445)\end{array}$ & $\begin{array}{c}(0.445 \\
0.668 \\
0.89)\end{array}$ & $\begin{array}{l}(0.238 \\
0.475 \\
0.713)\end{array}$ & $\begin{array}{c}(0.475 \\
0.713 \\
0.95)\end{array}$ \\
\hline P5 & $\begin{array}{l}(0.238, \\
0.475 \\
0.713)\end{array}$ & $\begin{array}{l}(0.158 \\
0.315 \\
0.473)\end{array}$ & $\begin{array}{c}(0,0 \\
0.158)\end{array}$ & $\begin{array}{l}(0.193 \\
0.385 \\
0.578)\end{array}$ & $\begin{array}{c}(0,0.158 \\
0.315)\end{array}$ & $\begin{array}{c}(0,0, \\
0.238)\end{array}$ & $\begin{array}{c}(0.355 \\
0.533 \\
0.71)\end{array}$ & $\begin{array}{c}(0.5, \\
0.75,1)\end{array}$ & $\begin{array}{c}(0,0.193 \\
0.385)\end{array}$ & $\begin{array}{l}(0.238, \\
0.475 \\
0.713)\end{array}$ & $\begin{array}{c}(0,0.193 \\
0.385)\end{array}$ & $\begin{array}{c}(0,0, \\
0.223)\end{array}$ \\
\hline P6 & $\begin{array}{c}(0.533 \\
0.71 \\
0.71)\end{array}$ & $\begin{array}{c}(0.578 \\
0.77 \\
0.77)\end{array}$ & $\begin{array}{c}(0.445 \\
0.668 \\
0.89)\end{array}$ & $\begin{array}{c}(0,0, \\
0.238)\end{array}$ & $\begin{array}{l}(0.178 \\
0.355 \\
0.533)\end{array}$ & $\begin{array}{c}(0.385 \\
0.578 \\
0.77)\end{array}$ & $\begin{array}{c}(0,0.178 \\
0.355)\end{array}$ & $\begin{array}{c}(0.475 \\
0.713 \\
0.95)\end{array}$ & $\begin{array}{c}(0.355 \\
0.533 \\
0.71)\end{array}$ & $\begin{array}{c}(0,0.178 \\
0.355)\end{array}$ & $\begin{array}{c}(0.475 \\
0.713 \\
0.95)\end{array}$ & $\begin{array}{l}(0.193 \\
0.385 \\
0.578)\end{array}$ \\
\hline$P 7$ & $\begin{array}{c}(0,0, \\
0.193)\end{array}$ & $\begin{array}{l}(0.193 \\
0.385 \\
0.578)\end{array}$ & $\begin{array}{c}(0.5 \\
0.75,1)\end{array}$ & $\begin{array}{l}(0.238 \\
0.475 \\
0.713)\end{array}$ & $\begin{array}{l}(0.223 \\
0.445 \\
0.668)\end{array}$ & $\begin{array}{c}(0,0.193 \\
0.385)\end{array}$ & $\begin{array}{c}(0,0.193 \\
0.385)\end{array}$ & $\begin{array}{c}(0.5, \\
0.75,1)\end{array}$ & $\begin{array}{c}(0,0, \\
0.158)\end{array}$ & $\begin{array}{l}(0.158 \\
0.315 \\
0.473)\end{array}$ & $\begin{array}{c}(0,0.193 \\
0.385)\end{array}$ & $\begin{array}{c}(0,0.178 \\
0.355)\end{array}$ \\
\hline$P 8$ & $\begin{array}{c}(0.445 \\
0.668 \\
0.89)\end{array}$ & $\begin{array}{c}(0.475 \\
0.713 \\
0.95)\end{array}$ & $\begin{array}{c}(0.75,1 \\
1)\end{array}$ & $\begin{array}{c}(0.668 \\
0.89 \\
0.89)\end{array}$ & $\begin{array}{c}(0.5, \\
0.75,1)\end{array}$ & $\begin{array}{c}(0.475 \\
0.713 \\
0.95)\end{array}$ & $\begin{array}{c}(0.475 \\
0.713 \\
0.95)\end{array}$ & $\begin{array}{c}(0.713 \\
0.95 \\
0.95)\end{array}$ & $\begin{array}{c}(0.475 \\
0.713 \\
0.95)\end{array}$ & $\begin{array}{c}(0.475 \\
0.713 \\
0.95)\end{array}$ & $\begin{array}{c}(0.475 \\
0.713 \\
0.95)\end{array}$ & $\begin{array}{c}(0.445 \\
0.668 \\
0.89)\end{array}$ \\
\hline$P 9$ & $\begin{array}{l}(0.1 \\
0.3 \\
0.53\end{array}$ & $\begin{array}{l}(0.223, \\
0.445, \\
0.668)\end{array}$ & $\begin{array}{c}(0.385 \\
0.578 \\
0.77)\end{array}$ & $\begin{array}{c}(0.475, \\
0.713, \\
0.95)\end{array}$ & $\begin{array}{l}(0.223, \\
0.445, \\
0.668)\end{array}$ & $\begin{array}{c}(0.315, \\
0.473, \\
0.63)\end{array}$ & $\begin{array}{c}(0.385 \\
0.578 \\
0.77)\end{array}$ & $\begin{array}{c}(0.315, \\
0.473, \\
0.63)\end{array}$ & $\begin{array}{l}(0.475, \\
0.713, \\
0.95)\end{array}$ & $\begin{array}{c}(0.668, \\
0.89 \\
0.89)\end{array}$ & $\begin{array}{c}(0.473, \\
0.63 \\
0.63)\end{array}$ & $\begin{array}{c}(0.315, \\
0.473, \\
0.63)\end{array}$ \\
\hline$P 10$ & $\begin{array}{l}(0.238, \\
0.475, \\
0.713)\end{array}$ & $\begin{array}{l}(0,0, \\
0.25)\end{array}$ & $\begin{array}{c}(0.25 \\
0.5,0.75)\end{array}$ & $\begin{array}{l}(0.223, \\
0.445, \\
0.668)\end{array}$ & $\begin{array}{c}(0,0.223 \\
0.445)\end{array}$ & $\begin{array}{l}(0.223, \\
0.445, \\
0.668)\end{array}$ & $\begin{array}{c}(0.25 \\
0.5,0.75)\end{array}$ & $\begin{array}{c}(0,0.238 \\
0.475)\end{array}$ & $\begin{array}{c}(0.25 \\
0.5,0.75)\end{array}$ & $\begin{array}{c}(0,0.223 \\
0.445)\end{array}$ & $\begin{array}{c}(0,0, \\
0.238)\end{array}$ & $\begin{array}{c}(0,0, \\
0.238)\end{array}$ \\
\hline$P 11$ & $\begin{array}{c}(0.713, \\
0.95 \\
0.95)\end{array}$ & $\begin{array}{c}(0,0.25 \\
0.5)\end{array}$ & $\begin{array}{c}(0.445, \\
0.668 \\
0.89)\end{array}$ & $\begin{array}{c}(0,0.238 \\
0.475)\end{array}$ & $\begin{array}{c}(0.533, \\
0.71 \\
0.71)\end{array}$ & $\begin{array}{c}(0,0.178 \\
0.355)\end{array}$ & $\begin{array}{l}(0.238, \\
0.475, \\
0.713)\end{array}$ & $\begin{array}{c}(0,0.223 \\
0.445)\end{array}$ & $\begin{array}{c}(0.385, \\
0.578, \\
0.77)\end{array}$ & $\begin{array}{c}(0.385, \\
0.578, \\
0.77)\end{array}$ & $\begin{array}{l}(0.178, \\
0.355, \\
0.533)\end{array}$ & $\begin{array}{c}(0,0.25 \\
0.5)\end{array}$ \\
\hline$P 12$ & $\begin{array}{l}(0.178, \\
0.355, \\
0.533)\end{array}$ & $\begin{array}{c}(0,0.223 \\
0.445)\end{array}$ & $\begin{array}{c}(0.223 \\
0.445 \\
0.668)\end{array}$ & $\begin{array}{l}(0.223, \\
0.445, \\
0.668)\end{array}$ & $\begin{array}{c}(0.385, \\
0.578, \\
0.77)\end{array}$ & $\begin{array}{c}(0,0.223 \\
0.445)\end{array}$ & $\begin{array}{c}(0.445, \\
0.668, \\
0.89)\end{array}$ & $\begin{array}{l}(0.223, \\
0.445, \\
0.668)\end{array}$ & $\begin{array}{c}(0,0.223 \\
0.445)\end{array}$ & $\begin{array}{c}(0,0.223 \\
0.445)\end{array}$ & $\begin{array}{c}(0.445, \\
0.668, \\
0.89)\end{array}$ & $\begin{array}{l}(0.193, \\
0.385, \\
0.578)\end{array}$ \\
\hline$P 13$ & $\begin{array}{c}(0,0, \\
0.223)\end{array}$ & $\begin{array}{c}(0,0, \\
0.178)\end{array}$ & $\begin{array}{c}(0.385 \\
0.578 \\
0.77)\end{array}$ & $\begin{array}{c}(0,0.238 \\
0.475)\end{array}$ & $\begin{array}{c}(0.445, \\
0.668, \\
0.89)\end{array}$ & $\begin{array}{l}(0,0, \\
0.193)\end{array}$ & $\begin{array}{c}(0,0.238 \\
0.475)\end{array}$ & $\begin{array}{l}(0.223, \\
0.445 \\
0.668)\end{array}$ & $\begin{array}{c}(0,0, \\
0.223)\end{array}$ & $\begin{array}{c}(0.385, \\
0.578, \\
0.77)\end{array}$ & $\begin{array}{c}(0,0.193 \\
0.385)\end{array}$ & $\begin{array}{c}(0.355 \\
0.533 \\
0.71)\end{array}$ \\
\hline P14 & $\begin{array}{l}(0.223, \\
0.445, \\
0.668)\end{array}$ & $\begin{array}{l}(0.178, \\
0.355, \\
0.533)\end{array}$ & $\begin{array}{c}(0.5 \\
0.75,1)\end{array}$ & $\begin{array}{l}(0.223, \\
0.445, \\
0.668)\end{array}$ & $\begin{array}{c}(0.445 \\
0.668 \\
0.89)\end{array}$ & $\begin{array}{c}(0,0.238 \\
0.475)\end{array}$ & $\begin{array}{l}(0,0 \\
0.25)\end{array}$ & $\begin{array}{c}(0,0.25 \\
0.5)\end{array}$ & $\begin{array}{c}(0,0 \\
0.178)\end{array}$ & $\begin{array}{l}(0.223, \\
0.445, \\
0.668)\end{array}$ & $\begin{array}{c}(0.385, \\
0.578, \\
0.77)\end{array}$ & $\begin{array}{l}(0.193, \\
0.385, \\
0.578)\end{array}$ \\
\hline$P 15$ & $\begin{array}{l}(0.238, \\
0.475, \\
0.713)\end{array}$ & $\begin{array}{l}(0,0 \\
0.25)\end{array}$ & $\begin{array}{c}(0.25 \\
0.5,0.75)\end{array}$ & $\begin{array}{c}(0,0, \\
0.223)\end{array}$ & $\begin{array}{c}(0,0.238 \\
0.475)\end{array}$ & $\begin{array}{c}(0.5, \\
0.75,1)\end{array}$ & $\begin{array}{c}(0,0.223 \\
0.445)\end{array}$ & $\begin{array}{c}(0.713 \\
0.95 \\
0.95)\end{array}$ & $\begin{array}{c}(0.445, \\
0.668, \\
0.89)\end{array}$ & $\begin{array}{c}(0,0.223 \\
0.445)\end{array}$ & $\begin{array}{c}(0,0.223 \\
0.445)\end{array}$ & $\begin{array}{c}(0.668 \\
0.89 \\
0.89)\end{array}$ \\
\hline P16 & $\begin{array}{c}(0.445, \\
0.668, \\
0.89)\end{array}$ & $\begin{array}{l}(0.238, \\
0.475, \\
0.713)\end{array}$ & $\begin{array}{l}(0.475 \\
0.713 \\
0.95)\end{array}$ & $\begin{array}{l}(0.193, \\
0.385, \\
0.578)\end{array}$ & $\begin{array}{l}(0.315, \\
0.473, \\
0.63)\end{array}$ & $\begin{array}{l}(0.1 \\
0.3 \\
0.53\end{array}$ & $\begin{array}{c}(0.578, \\
0.77 \\
0.77)\end{array}$ & $\begin{array}{l}(0.238, \\
0.475, \\
0.713)\end{array}$ & $\begin{array}{c}(0.25 \\
0.5,0.75)\end{array}$ & $\begin{array}{c}(0.533 \\
0.71 \\
0.71)\end{array}$ & & $\begin{array}{l}(0.178, \\
0.355, \\
0.533)\end{array}$ \\
\hline$P 17$ & $\begin{array}{c}(0,0.193 \\
0.385)\end{array}$ & $\begin{array}{c}(0.385 \\
0.578 \\
0.77)\end{array}$ & $\begin{array}{c}(0,0.178 \\
0.355)\end{array}$ & $\begin{array}{l}(0.178 \\
0.355 \\
0.533)\end{array}$ & $\begin{array}{c}(0.475 \\
0.713 \\
0.95)\end{array}$ & $\begin{array}{l}(0.238 \\
0.475 \\
0.713)\end{array}$ & $\begin{array}{l}(0.193 \\
0.385 \\
0.578)\end{array}$ & $\begin{array}{c}(0,0.238 \\
0.475)\end{array}$ & $\begin{array}{c}(0.475 \\
0.713 \\
0.95)\end{array}$ & $\begin{array}{c}(0,0.193 \\
0.385)\end{array}$ & $\begin{array}{l}(0.178 \\
0.355 \\
0.533)\end{array}$ & $\begin{array}{c}(0,0.178 \\
0.355)\end{array}$ \\
\hline$P 18$ & $\begin{array}{c}(0.25 \\
0.5,0.75)\end{array}$ & $\begin{array}{c}(0.475 \\
0.713 \\
0.95)\end{array}$ & $\begin{array}{c}(0.713 \\
0.95 \\
0.95)\end{array}$ & $\begin{array}{c}(0.355 \\
0.533 \\
0.71)\end{array}$ & $\begin{array}{c}(0.315 \\
0.473 \\
0.63)\end{array}$ & $\begin{array}{c}(0.25 \\
0.5,0.75)\end{array}$ & $\begin{array}{c}(0,0.25 \\
0.5)\end{array}$ & $\begin{array}{l}(0.178 \\
0.355 \\
0.533)\end{array}$ & $\begin{array}{c}(0,0.193 \\
0.385)\end{array}$ & $\begin{array}{c}(0.445 \\
0.668 \\
0.89)\end{array}$ & $\begin{array}{c}(0.158 \\
0.315 \\
0.473)\end{array}$ & $\begin{array}{c}(0.355 \\
0.533 \\
0.71)\end{array}$ \\
\hline$P 19$ & $\begin{array}{l}(0.193 \\
0.385 \\
0.578)\end{array}$ & $\begin{array}{c}(0,0 \\
0.178)\end{array}$ & $\begin{array}{l}(0,0, \\
0.178)\end{array}$ & $\begin{array}{c}(0.475 \\
0.713 \\
0.95)\end{array}$ & $\begin{array}{c}(0,0.25 \\
0.5)\end{array}$ & $\begin{array}{l}(0.178 \\
0.355 \\
0.533)\end{array}$ & $\begin{array}{c}(0.385 \\
0.578 \\
0.77)\end{array}$ & $\begin{array}{c}(0,0.193 \\
0.385)\end{array}$ & $\begin{array}{c}(0.475 \\
0.713 \\
0.95)\end{array}$ & $\begin{array}{c}(0,0 \\
0.223)\end{array}$ & $\begin{array}{l}(0.238 \\
0.475 \\
0.713)\end{array}$ & $\begin{array}{c}(0,0.238 \\
0.475)\end{array}$ \\
\hline$P 20$ & $\begin{array}{c}(0.75,1 \\
1)\end{array}$ & $\begin{array}{l}(0,0, \\
0.25)\end{array}$ & $\begin{array}{c}(0.445 \\
0.668 \\
0.89)\end{array}$ & $\begin{array}{l}(0.223, \\
0.445, \\
0.668)\end{array}$ & $\begin{array}{c}(0.445 \\
0.668 \\
0.89)\end{array}$ & $\begin{array}{c}(0.75,1 \\
1)\end{array}$ & $\begin{array}{l}(0.223, \\
0.445, \\
0.668)\end{array}$ & $\begin{array}{c}(0.475 \\
0.713 \\
0.95)\end{array}$ & $\begin{array}{c}(0,0, \\
0.238)\end{array}$ & $\begin{array}{c}(0,0.238 \\
0.475)\end{array}$ & $\begin{array}{l}(0.223, \\
0.445 \\
0.668)\end{array}$ & $\begin{array}{c}(0.25, \\
0.5,0.75)\end{array}$ \\
\hline
\end{tabular}


TABLE 48: Normalized scoring matrix.

\begin{tabular}{|c|c|c|c|c|c|c|c|c|c|c|c|c|}
\hline & $E 1$ & 2 & E3 & $E 4$ & S1 & $S 2$ & S3 & $\$ 4$ & G1 & G2 & G3 & G4 \\
\hline$P 1$ & $\begin{array}{l}0.037, \\
0.525, \\
0.762)\end{array}$ & $\begin{array}{c}(-0.392 \\
0.06,0.5)\end{array}$ & $\begin{array}{c}(0.065 \\
0.445 \\
0.668)\end{array}$ & $\begin{array}{c}(0,0.468 \\
0.765)\end{array}$ & $\begin{array}{l}(-0.222 \\
0,0.332)\end{array}$ & $\begin{array}{l}0.082, \\
0.555, \\
0.777)\end{array}$ & $\begin{array}{c}(-0.422 \\
0.02,0.5)\end{array}$ & $\begin{array}{l}(-0.287 \\
0.2,0.5)\end{array}$ & $\begin{array}{c}(0.592,1 \\
1)\end{array}$ & $\begin{array}{c}(-0.297 \\
0.186 \\
0.5)\end{array}$ & $\begin{array}{c}0.527,1 \\
1)\end{array}$ & $\begin{array}{c}(-0.047 \\
0.437 \\
0.749)\end{array}$ \\
\hline$P 2$ & $\begin{array}{c}(0.305 \\
0.777,1)\end{array}$ & $\begin{array}{c}(-0.202 \\
0.202 \\
0.595)\end{array}$ & $\begin{array}{c}(-0.158 \\
0.223 \\
0.445)\end{array}$ & $\begin{array}{c}(0.5, \\
0.937,1)\end{array}$ & $\begin{array}{c}(0.283, \\
0.697,1)\end{array}$ & $\begin{array}{l}(0.037 \\
0.525 \\
0.762)\end{array}$ & $\begin{array}{c}(-0.312 \\
0.102 \\
0.555)\end{array}$ & $\begin{array}{l}(0.18, \\
0.595 \\
0.822)\end{array}$ & $\begin{array}{l}(-0.158 \\
0,0.223)\end{array}$ & $\begin{array}{c}(-0.297 \\
0.186 \\
0.5)\end{array}$ & $\begin{array}{l}(-0.223 \\
0,0.223)\end{array}$ & $\begin{array}{c}(0.5, \\
0.937,1)\end{array}$ \\
\hline P3 & $\begin{array}{c}(-0.02 \\
0.23 \\
0.422)\end{array}$ & $\begin{array}{c}(0.277, \\
0.644,1)\end{array}$ & $\begin{array}{c}(0.197 \\
0.533 \\
0.71)\end{array}$ & $\begin{array}{c}(-0.107 \\
0.126 \\
0.392)\end{array}$ & $\begin{array}{c}(-0.042 \\
0.357 \\
0.645)\end{array}$ & $\begin{array}{c}(-0.02 \\
0.422 \\
0.615)\end{array}$ & $\begin{array}{c}(0.133, \\
0.547,1)\end{array}$ & $\begin{array}{c}(0.358, \\
0.772,1)\end{array}$ & $\begin{array}{c}(-0.158 \\
0.193 \\
0.385)\end{array}$ & $\begin{array}{l}(0.04 \\
0.274 \\
0.502)\end{array}$ & $\begin{array}{c}(-0.223 \\
0.193 \\
0.385)\end{array}$ & $\begin{array}{c}(-0.234 \\
0.234 \\
0.532)\end{array}$ \\
\hline$P 4$ & $\begin{array}{l}(-0.2 \\
0.287 \\
0.525)\end{array}$ & $\begin{array}{c}(0.14, \\
0.576,1)\end{array}$ & $\begin{array}{c}(0.287 \\
0.668 \\
0.89)\end{array}$ & $\begin{array}{c}(0,0.468 \\
0.765)\end{array}$ & $\begin{array}{c}(0.418 \\
0.89,1)\end{array}$ & $\begin{array}{c}(-0.25 \\
0.25 \\
0.5)\end{array}$ & $\begin{array}{c}(-0.135 \\
0.295 \\
0.762)\end{array}$ & $\begin{array}{c}(-0.037 \\
0.45 \\
0.75)\end{array}$ & $\begin{array}{r}(-0 . \\
0.2 \\
0.4\end{array}$ & $\begin{array}{c}(-0.234 \\
0.234 \\
0.532)\end{array}$ & $\begin{array}{l}(0 . \\
0 . \\
0 .\end{array}$ & $\begin{array}{c}(-0.297 \\
0.186 \\
0.5)\end{array}$ \\
\hline P5 & $\begin{array}{l}(0.037, \\
0.525, \\
0.762)\end{array}$ & $\begin{array}{l}(0.111, \\
0.479 \\
0.834)\end{array}$ & $\begin{array}{l}(-0.158 \\
0,0.158)\end{array}$ & $\begin{array}{l}(0.095, \\
0.532, \\
0.797)\end{array}$ & $\begin{array}{c}(0.353, \\
0.732,1)\end{array}$ & $\begin{array}{c}(0.512, \\
1,1)\end{array}$ & $\begin{array}{c}(-0.132 \\
0.237 \\
0.645)\end{array}$ & $\begin{array}{l}(-0.287 \\
0.2,0.5)\end{array}$ & $\begin{array}{r}(-0 . \\
0.1 \\
0.3\end{array}$ & $\begin{array}{c}(-0.047 \\
0.437 \\
0.749)\end{array}$ & $\begin{array}{c}(-0 \\
0 . \\
0.3\end{array}$ & $\begin{array}{c}(0.468, \\
0.937,1)\end{array}$ \\
\hline P6 & $\begin{array}{c}(0.04, \\
0.29, \\
0.467)\end{array}$ & $\begin{array}{l}(-0.202 \\
0,0.392)\end{array}$ & $\begin{array}{c}(0.287, \\
0.668, \\
0.89)\end{array}$ & $\begin{array}{c}(0.453, \\
0.937,1)\end{array}$ & $\begin{array}{l}(0.135, \\
0.535, \\
0.822)\end{array}$ & $\begin{array}{l}(-0.02, \\
0.422 \\
0.615)\end{array}$ & $\begin{array}{c}(0.223, \\
0.592,1)\end{array}$ & $\begin{array}{c}(-0.237 \\
0.237 \\
0.525)\end{array}$ & $\begin{array}{c}(0.197 \\
0.533 \\
0.71)\end{array}$ & $\begin{array}{c}(0.329, \\
0.749,1)\end{array}$ & & $\begin{array}{l}(0.095, \\
0.532, \\
0.797)\end{array}$ \\
\hline$P 7$ & $\begin{array}{c}(0.557 \\
1,1)\end{array}$ & $\begin{array}{c}(0,0.405 \\
0.797)\end{array}$ & $\begin{array}{c}(0.342 \\
0.75,1)\end{array}$ & $\begin{array}{c}(-0.047 \\
0.437 \\
0.749)\end{array}$ & $\begin{array}{c}(0,0.445) \\
0.777)\end{array}$ & $\begin{array}{c}(0.365 \\
0.807,1)\end{array}$ & $\begin{array}{c}(0.193, \\
0.577,1)\end{array}$ & $\begin{array}{l}(-0.287 \\
0.2,0.5)\end{array}$ & & & $\begin{array}{l}23, \\
3, \\
5)\end{array}$ & $\begin{array}{c}(0.329, \\
0.749,1)\end{array}$ \\
\hline$P 8$ & $\begin{array}{l}(-0.14, \\
0.332, \\
0.555)\end{array}$ & $\begin{array}{c}(-0.392 \\
0.06,0.5)\end{array}$ & $\begin{array}{c}(0.592,1, \\
1)\end{array}$ & $\begin{array}{l}(-0.234 \\
0,0.297)\end{array}$ & $\begin{array}{c}(-0.332, \\
0.14,0.5)\end{array}$ & $\begin{array}{l}(-0.2 \\
0.287 \\
0.525)\end{array}$ & $\begin{array}{c}(-0.372 \\
0.057 \\
0.525)\end{array}$ & $\begin{array}{l}(-0.237 \\
0,0.287)\end{array}$ & & $\begin{array}{c}(-0.297 \\
0.186 \\
0.5)\end{array}$ & & $\begin{array}{c}(-0.234 \\
0.234 \\
0.532)\end{array}$ \\
\hline$P 9$ & $\begin{array}{l}(0.2 \\
0.6 \\
0.8\end{array}$ & $\begin{array}{c}(-0.095 \\
0.342 \\
0.765)\end{array}$ & $\begin{array}{c}(0.227, \\
0.578, \\
0.77)\end{array}$ & $\begin{array}{c}(-0.297 \\
0.186 \\
0.5)\end{array}$ & $\begin{array}{c}(0,0.445 \\
0.777)\end{array}$ & $\begin{array}{l}(0.12, \\
0.527 \\
0.685)\end{array}$ & $\begin{array}{c}(-0.192 \\
0.192 \\
0.615)\end{array}$ & $\begin{array}{l}(0.083, \\
0.477, \\
0.685)\end{array}$ & $\begin{array}{l}(0.317, \\
0.713, \\
0.95)\end{array}$ & $\begin{array}{l}(-0.234 \\
0,0.297)\end{array}$ & $\begin{array}{l}(0.25 \\
0.63 \\
0.63)\end{array}$ & $\begin{array}{l}(0.04, \\
0.439, \\
0.668)\end{array}$ \\
\hline$P 10$ & $\begin{array}{l}(0.0 \\
0.5 \\
0.7\end{array}$ & $\begin{array}{c}(0.345 \\
0.811,1)\end{array}$ & $\begin{array}{c}(0.092, \\
0.5,0.75)\end{array}$ & $\begin{array}{c}(0,0.468 \\
0.765)\end{array}$ & $\begin{array}{c}(0.223, \\
0.667,1)\end{array}$ & $\begin{array}{l}(0.082, \\
0.555, \\
0.777)\end{array}$ & $\begin{array}{c}(-0.172 \\
0.27 \\
0.75)\end{array}$ & $\begin{array}{c}(0.238, \\
0.712,1)\end{array}$ & $\begin{array}{c}(0.092 \\
0.5,0.75)\end{array}$ & $\begin{array}{c}(0.235, \\
0.702,1)\end{array}$ & & $\begin{array}{c}(0.453, \\
0.937,1)\end{array}$ \\
\hline$P 11$ & $\begin{array}{c}(-0.2, \\
0.05, \\
0.287)\end{array}$ & $\begin{array}{c}(0.082, \\
0.547,1)\end{array}$ & $\begin{array}{c}(0.287, \\
0.668, \\
0.89)\end{array}$ & $\begin{array}{c}(0.203, \\
0.686,1)\end{array}$ & $\begin{array}{c}(-0.042 \\
0.18 \\
0.467)\end{array}$ & $\begin{array}{c}(0.395 \\
0.822,1)\end{array}$ & $\begin{array}{c}(-0.135 \\
0.295 \\
0.762)\end{array}$ & $\begin{array}{c}(0.268, \\
0.727,1)\end{array}$ & & $\begin{array}{c}(-0.107 \\
0.328 \\
0.595)\end{array}$ & $\begin{array}{l}045, \\
55, \\
33)\end{array}$ & $\begin{array}{c}(0.177 \\
0.674,1)\end{array}$ \\
\hline$P 12$ & $\begin{array}{l}(0.217, \\
0.645 \\
0.822)\end{array}$ & $\begin{array}{c}(0.14, \\
0.576,1)\end{array}$ & $\begin{array}{l}(0.065, \\
0.445, \\
0.668)\end{array}$ & $\begin{array}{c}(0,0.468 \\
0.765)\end{array}$ & $\begin{array}{c}(-0.102 \\
0.312 \\
0.615)\end{array}$ & $\begin{array}{c}(0.305 \\
0.777,1)\end{array}$ & $\begin{array}{c}(-0.312 \\
0.102 \\
0.555)\end{array}$ & $\begin{array}{l}(0.045, \\
0.505, \\
0.777)\end{array}$ & $\begin{array}{r}(-0.1 \\
0.22 \\
0.44\end{array}$ & $\begin{array}{c}(0.235, \\
0.702,1)\end{array}$ & & \\
\hline$P 13$ & $\begin{array}{c}(0.527 \\
1,1)\end{array}$ & $\begin{array}{c}(0.421, \\
0.811,1)\end{array}$ & $\begin{array}{c}(0.227 \\
0.578 \\
0.77)\end{array}$ & $\begin{array}{c}(0.203, \\
0.686,1)\end{array}$ & $\begin{array}{r}(-0.2 \\
0.22 \\
0.55\end{array}$ & $\begin{array}{c}(0.557 \\
1,1)\end{array}$ & $\begin{array}{c}(0.103, \\
0.532,1)\end{array}$ & $\begin{array}{l}(0.045, \\
0.505, \\
0.777)\end{array}$ & $\begin{array}{l}(-0.158 \\
0,0.223)\end{array}$ & $\begin{array}{r}(-0 . \\
0.3 \\
0.5\end{array}$ & $\begin{array}{l}23 \\
5\end{array}$ & $\begin{array}{c}(-0.044 \\
0.376 \\
0.626)\end{array}$ \\
\hline$P 14$ & & $\begin{array}{l}(0.047, \\
0.437, \\
0.813)\end{array}$ & $\begin{array}{c}(0.342, \\
0.75,1)\end{array}$ & $\begin{array}{c}(0,0.468 \\
0.765)\end{array}$ & $\begin{array}{c}(-0.222 \\
0.222 \\
0.555)\end{array}$ & $\begin{array}{c}(0.275 \\
0.762,1)\end{array}$ & $\begin{array}{c}(0.328 \\
0.77,1)\end{array}$ & & & $\begin{array}{c}(0,0.468 \\
0.765)\end{array}$ & & $\begin{array}{l}(0.095, \\
0.532, \\
0.797)\end{array}$ \\
\hline$P 15$ & $\begin{array}{l}.037 \\
.525 \\
762)\end{array}$ & $\begin{array}{c}(0.345, \\
0.811,1)\end{array}$ & $\begin{array}{c}(0.092, \\
0.5,0.75)\end{array}$ & $\begin{array}{c}(0.468, \\
0.937,1)\end{array}$ & $\begin{array}{c}(0.193, \\
0.652,1)\end{array}$ & $\begin{array}{c}(-0.25 \\
0.25 \\
0.5)\end{array}$ & $\begin{array}{c}(0.133, \\
0.547,1)\end{array}$ & $\begin{array}{l}(-0.237 \\
0,0.287)\end{array}$ & $\begin{array}{c}(0.287, \\
0.668, \\
0.89)\end{array}$ & $\begin{array}{c}(0.235, \\
0.702,1)\end{array}$ & $\begin{array}{l}23, \\
3, \\
5)\end{array}$ & $\begin{array}{l}(-0.234 \\
0,0.297)\end{array}$ \\
\hline$P 16$ & $\begin{array}{l}(-0.14, \\
0.332, \\
0.555)\end{array}$ & $\begin{array}{c}(-0.142 \\
0.311 \\
0.749)\end{array}$ & $\begin{array}{c}(0.317 \\
0.713 \\
0.95)\end{array}$ & $\begin{array}{l}(0.095, \\
0.532, \\
0.797)\end{array}$ & $\begin{array}{c}(0.038 \\
0.417 \\
0.685)\end{array}$ & $\begin{array}{l}(0.217 \\
0.645 \\
0.822)\end{array}$ & $\begin{array}{l}(-0.192 \\
0,0.422)\end{array}$ & $\begin{array}{c}(0,0.475 \\
0.762)\end{array}$ & $\begin{array}{c}(0.092, \\
0.5,0.75)\end{array}$ & $\begin{array}{c}(-0.044 \\
0.189 \\
0.439)\end{array}$ & $\begin{array}{r}(-0 \\
0.3 \\
0.5\end{array}$ & $\begin{array}{l}(0.142, \\
0.563, \\
0.813)\end{array}$ \\
\hline$P 17$ & $\begin{array}{c}(0.365 \\
0.807,1)\end{array}$ & $\begin{array}{c}(-0.202 \\
0.202 \\
0.595)\end{array}$ & $\begin{array}{c}(-0.158 \\
0.178 \\
0.355)\end{array}$ & $\begin{array}{l}(0.142 \\
0.563 \\
0.813)\end{array}$ & $\begin{array}{c}(-0.282 \\
0.177 \\
0.525)\end{array}$ & $\begin{array}{l}(0.037, \\
0.525, \\
0.762)\end{array}$ & $\begin{array}{c}(0,0.385 \\
0.807)\end{array}$ & $\begin{array}{c}(0.238, \\
0.712,1)\end{array}$ & & & $\begin{array}{c}(-0.045 \\
0.355 \\
0.533)\end{array}$ & $\begin{array}{c}(0.329 \\
0.749,1)\end{array}$ \\
\hline$P 18$ & $\begin{array}{c}(0,0.5, \\
0.75)\end{array}$ & $\begin{array}{c}(-0.392, \\
0.06,0.5)\end{array}$ & $\begin{array}{c}(0.555 \\
0.95 \\
0.95)\end{array}$ & $\begin{array}{c}(-0.044 \\
0.376 \\
0.626)\end{array}$ & $\begin{array}{c}(0.038 \\
0.417 \\
0.685)\end{array}$ & $\begin{array}{c}(0,0.5 \\
0.75)\end{array}$ & $\begin{array}{c}(0.078 \\
0.52,1)\end{array}$ & $\begin{array}{l}(0.18, \\
0.595, \\
0.822)\end{array}$ & $\begin{array}{c}(-0.158 \\
0.193 \\
0.385)\end{array}$ & $\begin{array}{c}(-0.234 \\
0.234 \\
0.532)\end{array}$ & $\begin{array}{c}(-0.065 \\
0.315 \\
0.473)\end{array}$ & $\begin{array}{c}(-0.044 \\
0.376 \\
0.626)\end{array}$ \\
\hline P19 & $\begin{array}{l}(0.172, \\
0.615, \\
0.807)\end{array}$ & $\begin{array}{c}(0.421, \\
0.811,1)\end{array}$ & $\begin{array}{l}(-0.158 \\
0,0.178)\end{array}$ & $\begin{array}{c}(-0.297 \\
0.186 \\
0.5)\end{array}$ & $\begin{array}{c}(0.168 \\
0.64,1)\end{array}$ & $\begin{array}{l}(0.217 \\
0.645 \\
0.822)\end{array}$ & $\begin{array}{c}(-0.192 \\
0.192 \\
0.615)\end{array}$ & $\begin{array}{c}(0.328, \\
0.757,1)\end{array}$ & $\begin{array}{c}(0.317 \\
0.713 \\
0.95)\end{array}$ & $\begin{array}{c}(0.468, \\
0.937,1)\end{array}$ & $\begin{array}{l}(0.015 \\
0.475 \\
0.713)\end{array}$ & $\begin{array}{c}(0.203, \\
0.686,1)\end{array}$ \\
\hline$P 20$ & $\begin{array}{l}(-0.25 \\
0,0.25)\end{array}$ & $\begin{array}{c}(0.345, \\
0.811,1)\end{array}$ & $\begin{array}{c}(0.287, \\
0.668, \\
0.89)\end{array}$ & $\begin{array}{c}(0,0.468 \\
0.765)\end{array}$ & $\begin{array}{c}(-0.222 \\
0.222 \\
0.555)\end{array}$ & $\begin{array}{l}(-0.25 \\
0,0.25)\end{array}$ & $\begin{array}{c}(-0.09 \\
0.325 \\
0.777)\end{array}$ & $\begin{array}{c}(-0.237 \\
0.237 \\
0.525)\end{array}$ & $\begin{array}{l}(-0.158 \\
0,0.238)\end{array}$ & $\begin{array}{c}(0.203, \\
0.686,1)\end{array}$ & $\begin{array}{c}(0,0.445 \\
0.668)\end{array}$ & $\begin{array}{c}(-0.086 \\
0.411 \\
0.737)\end{array}$ \\
\hline
\end{tabular}


TABle 49: Project ranking table (20 projects).

\begin{tabular}{|c|c|c|c|c|c|c|c|c|c|c|}
\hline Project & $S$ & $\widetilde{S}_{\text {def }}^{\mathrm{Z}}$ & $R$ & $\widetilde{R}_{\text {def }}^{\mathrm{Z}}$ & $Q$ & $\widetilde{Q}_{\mathrm{def}}^{Z}$ & $R$ & $S$ & $Q$ & Rank \\
\hline$P 1$ & $(-0.036,0.414,0.681)$ & 0.368 & $(0.053,0.09,0.09)$ & 0.081 & $(-0.487,0.258,0.831)$ & 0.215 & 13 & 7 & 11 & 11 \\
\hline$P 2$ & $(0.016,0.411,0.664)$ & 0.375 & $(0.052,0.096,0.109)$ & 0.088 & $(-0.469,0.291,0.924)$ & 0.26 & 17 & 8 & 13 & 13 \\
\hline$P 3$ & $(0.016,0.365,0.626)$ & 0.343 & $(0.027,0.064,0.109)$ & 0.066 & $(-0.599,0.09,0.904)$ & 0.121 & 7 & 3 & 5 & 5 \\
\hline$P 4$ & $(-0.065,0.389,0.685)$ & 0.35 & $(0.019,0.057,0.099)$ & 0.058 & $(-0.689,0.067,0.881)$ & 0.081 & 2 & 4 & 3 & 3 \\
\hline$P 5$ & $(0.026,0.442,0.686)$ & 0.399 & $(0.037,0.072,0.083)$ & 0.066 & $(-0.543,0.176,0.796)$ & 0.151 & 6 & 10 & 7 & 7 \\
\hline P6 & $(0.139,0.52,0.766)$ & 0.486 & $(0.047,0.096,0.109)$ & 0.087 & $(-0.429,0.35,0.979)$ & 0.312 & 15 & 16 & 17 & 17 \\
\hline P7 & $(0.11,0.515,0.765)$ & 0.476 & $(0.061,0.109,0.109)$ & 0.097 & $(-0.369,0.415,0.978)$ & 0.36 & 20 & 13 & 19 & 19 \\
\hline$P 8$ & $(-0.129,0.288,0.583)$ & 0.258 & $(0.031,0.064,0.086)$ & 0.061 & $(-0.658,0.051,0.754)$ & 0.049 & 3 & 1 & 1 & 1 \\
\hline$P 9$ & $(0.017,0.41,0.667)$ & 0.376 & $(0.029,0.07,0.09)$ & 0.065 & $(-0.593,0.15,0.821)$ & 0.132 & 5 & 9 & 6 & 6 \\
\hline$P 10$ & $(0.098,0.536,0.806)$ & 0.494 & $(0.034,0.08,0.106)$ & 0.075 & $(-0.519,0.271,0.984)$ & 0.252 & 10 & 19 & 12 & 12 \\
\hline$P 11$ & $(0.068,0.473,0.763)$ & 0.444 & $(0.028,0.071,0.103)$ & 0.068 & $(-0.566,0.185,0.945)$ & 0.187 & 8 & 11 & 9 & 9 \\
\hline$P 12$ & $(0.063,0.499,0.787)$ & 0.462 & $(0.025,0.074,0.106)$ & 0.07 & $(-0.588,0.219,0.974)$ & 0.206 & 9 & 12 & 10 & 10 \\
\hline$P 13$ & $(0.128,0.539,0.765)$ & 0.493 & $(0.057,0.109,0.109)$ & 0.096 & $(-0.377,0.427,0.978)$ & 0.364 & 19 & 17 & 20 & 20 \\
\hline$P 14$ & $(0.097,0.52,0.782)$ & 0.48 & $(0.036,0.084,0.109)$ & 0.078 & $(-0.511,0.282,0.988)$ & 0.26 & 12 & 15 & 14 & 14 \\
\hline$P 15$ & $(0.097,0.521,0.777)$ & 0.479 & $(0.048,0.096,0.109)$ & 0.088 & $(-0.443,0.35,0.984)$ & 0.311 & 16 & 14 & 16 & 16 \\
\hline$P 16$ & $(0.003,0.389,0.668)$ & 0.362 & $(0.016,0.055,0.082)$ & 0.052 & $(-0.665,0.054,0.781)$ & 0.056 & 1 & 6 & 2 & 2 \\
\hline$P 17$ & $(0.109,0.53,0.803)$ & 0.493 & $(0.04,0.088,0.109)$ & 0.081 & $(-0.483,0.309,0.999)$ & 0.284 & 14 & 18 & 15 & 15 \\
\hline$P 18$ & $(-0.04,0.393,0.665)$ & 0.353 & $(0.029,0.057,0.109)$ & 0.063 & $(-0.621,0.067,0.925)$ & 0.109 & 4 & 5 & 4 & 4 \\
\hline$P 19$ & $(0.138,0.561,0.801)$ & 0.515 & $(0.05,0.099,0.106)$ & 0.089 & $(-0.414,0.387,0.981)$ & 0.335 & 18 & 20 & 18 & 18 \\
\hline$P 20$ & $(-0.03,0.361,0.645)$ & 0.335 & $(0.034,0.08,0.106)$ & 0.075 & $(-0.587,0.177,0.898)$ & 0.166 & 10 & 2 & 8 & 8 \\
\hline
\end{tabular}

fuzzy DEMATEL variable, except that the probability of fuzzy numbers is assumed.

Step 3: create a primary fuzzy matrix of direct connection by collecting expert opinions.

To measure the relationships between the criteria, we need to put them in a square matrix and ask the experts to compare them in pairs based on their effect on each other. In this survey, experts will express their views based on Tables 4 and 5. Assume that we have $n$ criteria and $p$ expertise; we have $p$ fuzzy matrix, each of which corresponds to the views of an expert with triangular fuzzy numbers as its elements.

Step 4: normalize the fuzzy matrix of direct connection.

To this end, linear scale conversion is used as a normalization formula to convert criteria scales to comparable scales:

$$
\begin{aligned}
& \tilde{b}_{i j}^{z}=\sum_{j=1}^{n} \tilde{W}_{i j}^{z}=\left(\sum_{j=1}^{n} f_{i j}, \sum_{j=1}^{n} g_{i j}, \sum_{j=1}^{n} h_{i j}\right), \\
& h=\max _{1 \leq i \leq n}\left(\sum_{j=1}^{n} h_{i j}\right), \\
& \tilde{Y}^{Z}=\left[\begin{array}{ccc}
\tilde{Y}_{11}^{z} & \cdots & \tilde{Y}_{1 n}^{z} \\
\vdots & \ddots & \vdots \\
\tilde{Y}_{1 m}^{z} & \cdots & \tilde{Y}_{m n}^{z}
\end{array}\right], \\
& \tilde{Y}_{i j}^{z}=\frac{\tilde{W}_{i j}^{z}=\left(\frac{f_{i j}}{h}, \frac{g_{i j}}{h}, \frac{h_{i j}}{h}\right) .}{h}
\end{aligned}
$$

Step 5: calculate the fuzzy matrix of the total relationship.

In this step, we first calculate the inverse of the normal matrix, then subtract it from the matrix $I$, and finally multiply the normal matrix by the following resulting matrix equations:

$$
\begin{aligned}
& \widetilde{T}^{Z}=\widetilde{Y}^{Z} \times\left(I-\widetilde{Y}^{Z}\right)^{-1}, \\
& {\left[f_{i j}^{\prime \prime}\right]=Y_{f} \times\left(I-Y_{f}\right)^{-1},} \\
& {\left[g_{i j}^{\prime \prime}\right]=Y_{g} \times\left(I-Y_{g}\right)^{-1},} \\
& {\left[h_{i j}^{\prime \prime}\right]=Y_{h} \times\left(I-Y_{h}\right)^{-1} .}
\end{aligned}
$$

Therefore, the $\widetilde{T}^{Z}$ matrix is defined as follows:

$$
\widetilde{T}^{Z}=\left[\begin{array}{ccc}
\tilde{t}_{11}^{z} & \cdots & \tilde{t}_{1 n}^{z} \\
\vdots & \ddots & \vdots \\
\tilde{t}_{1 m}^{z} & \cdots & \tilde{t}_{m n}^{z}
\end{array}\right] .
$$

Step 6: create and analyze the causal diagram.

For this purpose, we first calculate the sum of the elements of each row $\left(\widetilde{D}_{i}^{z}\right)$ and the sum of the elements of each column $\left(\widetilde{R}_{j}^{z}\right)$ of the fuzzy matrix. The sum of the elements of each row $\left(\widetilde{D}^{z}\right)$ for each criterion indicates the effect of that criterion on other system criteria. The sum of the elements of the column $(\widetilde{R})$ for each criterion indicates the effectiveness of that criterion from 


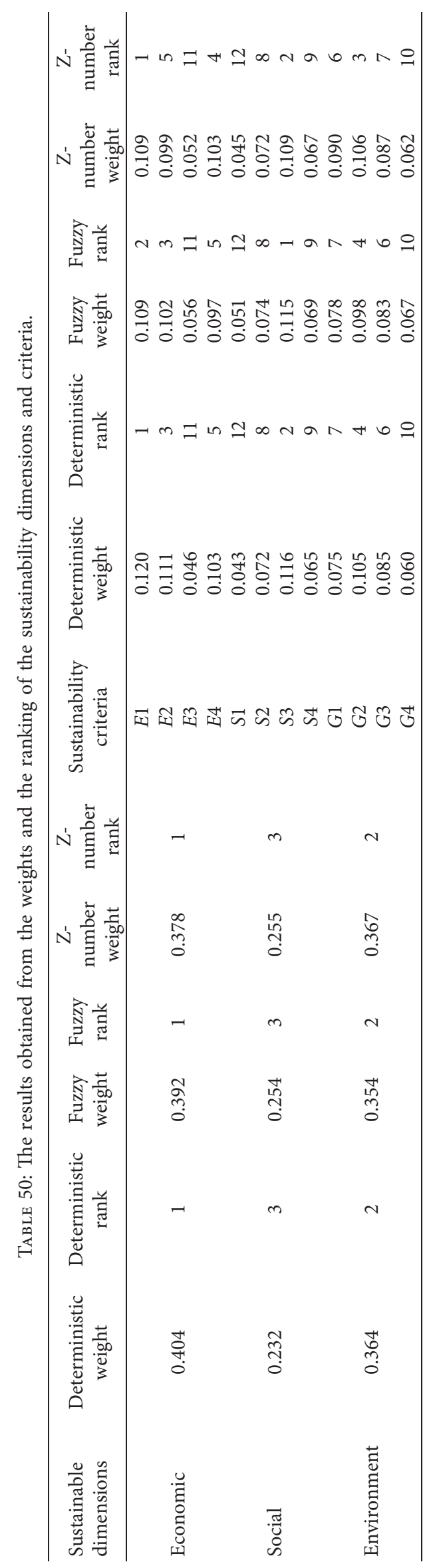


the other criteria of the system. Then, we easily get the values $\left(\widetilde{D}^{z}+\widetilde{R}^{z}\right)$ and $\left(\widetilde{D}^{z}-\widetilde{R}^{z}\right)$ :

$$
\begin{gathered}
\widetilde{D}_{i}^{z}=\sum_{j=1}^{n} n, \quad(i=1,2, \cdots, n), \\
\widetilde{R}_{j}^{z}=\sum_{i=1}^{n} \widetilde{t}_{i j}^{z}, \quad(j=1,2, \cdots, n), \\
\widetilde{D}_{i}^{z}+\widetilde{R}_{j}^{z}, \quad(i=1,2, \cdots, n),(j=1,2, \cdots, n), \\
\widetilde{D}_{i}^{z}-\widetilde{R}_{j}^{z}, \quad(i=1,2, \cdots, n),(j=1,2, \cdots, n) .
\end{gathered}
$$

To draw a causal diagram, we need to decode these two values, like the deterministic DEMATEL method. Here we use the weighted average method to defuzzy these two values:

$$
Z \text { - number defuzzy: } \frac{f+2 g+h}{4} .
$$

Therefore, the horizontal vector $\left(\widetilde{D}^{z}+\widetilde{R}^{z}\right)$ is the effect of the desired criteria on the system. In other words, the higher the value of the criteria $\left(\widetilde{D}^{z}+\widetilde{R}^{z}\right)$, the more it interacts with other criteria in the system.

The vertical vector $\left(\widetilde{D}^{z}-\widetilde{R}^{z}\right)$ shows the impact power of each criterion. In general, if $\left(\widetilde{D}^{z}-\widetilde{R}^{z}\right)$ is positive, the variable is a causal variable, and if it is negative, it is considered an effect variable.

After deactivating the numbers, a Cartesian coordinate system is drawn in which the longitudinal axis shows the values $\left(\widetilde{D}^{z}+\widetilde{R}^{z}\right)$ and $\left(\widetilde{D}^{z}-\widetilde{R}^{z}\right)$ are in the transverse axis.

Thus,

(i) The horizontal vector in the coordinate system is the amount of influence of the desired criteria on the system. In other words, the higher this value for a criterion, the more interaction it has with other system criteria.

(ii) The vertical vector of the coordinate system shows the effective power of each criterion. In general, if this value is positive for a criterion, it is considered a causal variable, and if it is negative, it is considered an effect variable.

Step 7: calculate the importance weights of the criteria. To calculate the weights of the dimensions, we use the following equation:

$$
W_{d}=\sqrt[2]{\left(\widetilde{D}_{d}^{z: \text { def }}+\widetilde{R}_{d}^{z \text { def }}\right)^{2}+\left(\widetilde{D}_{d}^{z: \text { def }}-\widetilde{R}_{d}^{z: \text { def }}\right)^{2}}
$$

where $W_{d}$ is the weight of the sustainability dimension. And the normalized weight of the sustainability dimensions is as follows:

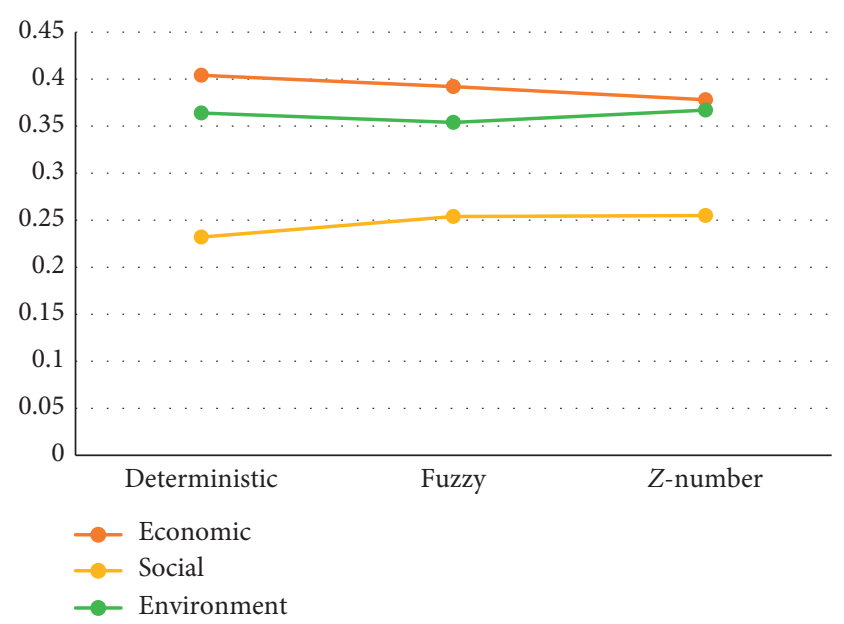

FIGURE 9: Comparison of importance weights of sustainability dimensions under different scenarios (in terms of quantity and ranking).

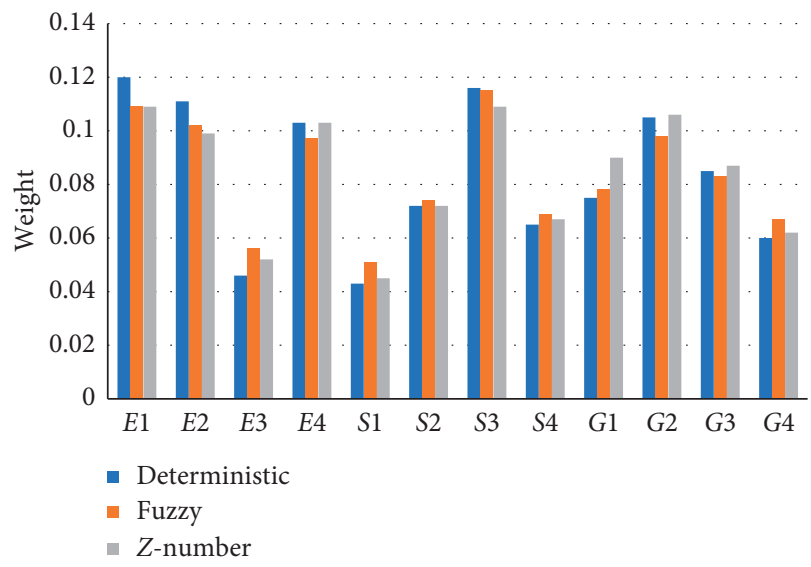

FIGURE 10: Comparison of importance weights of sustainability criteria under different scenarios (in terms of weight gain).

$$
W_{d}^{F}=\frac{w_{d}}{\sum_{i=1}^{m} w_{d}}
$$

where $W_{d}^{F}$ is the normalized weight of the sustainability dimension.

We also use the following formula to calculate the importance weights of the criteria:

$W_{\mathrm{id}}=W_{d}^{F} \times \sqrt[2]{\left(\widetilde{D}_{i}^{z: \mathrm{def}}+\widetilde{R}_{j}^{z \mathrm{def}}\right)^{2}+\left(\widetilde{D}_{i}^{z: \mathrm{def}}-\widetilde{R}_{j}^{z \mathrm{def}}\right)^{2}}$,

where $W_{\text {id }}$ is the weight of the sustainability criteria relative to its sustainability dimension.

And the normalized weight of the sustainability criteria is as follows:

$$
W_{\mathrm{id}}^{F}=\frac{w_{\mathrm{id}}}{\sum_{i=1}^{n} w_{\mathrm{id}}},
$$


where $W_{\text {id }}$ is the normalized weight of the sustainability criteria relative to its sustainability dimension.

3.3. Z-Number VIKOR Approach for Project Ranking. VIKOR is a multicriteria decision-making method. The ranking criteria in this method are based on their degree of closeness to the ideal answer. This approach can provide a maximum amount of group desirability for the majority and a minimum individual effect for the opposition. The steps in this method are similar to those of the VIKOR method, as described in the following article by Eprikovik.

\subsubsection{Fuzzy VIKOR Steps (Z-Number VIKOR)}

Step 1: form the decision matrix.

The decision matrix of this method is the same as the decision matrix of the TOPSIS method, i.e., the matrix includes the criteria option. Also, in this step, the weight of the criteria and the type of criteria (quantitative and qualitative, positive and negative) should be determined. The weight of the sustainability criteria was obtained in the previous step using the Z-DEMATEL approach. Also, the type of criteria is divided into two categories of positive and negative criteria. Positive criteria increase profits and improve the system, such as project profits. Negative criteria are those that by reduction, improve the system, such as the lower the cost, the better for the system. Table 2 shows the linguistic variables and their corresponding fuzzy numbers.

To calculate the linguistic variable of Z-number, we use equation (14) and an example of its calculation is as follows:

$$
\begin{aligned}
& \text { very small - unlikely, } \\
& \begin{aligned}
A^{\prime} & =[\sqrt{\alpha} d, \sqrt{\alpha} e, \sqrt{\alpha} f]=\left[d^{\prime}, e^{\prime}, f^{\prime}\right] \\
& =0.45 \times[0.00,0.00,0.25]=[0.00,0.00,0.11]
\end{aligned}
\end{aligned}
$$

Finally, in Table 7, the linguistic variable of Z-number is prepared. As can be seen, this variable is similar to the fuzzy DEMATEL variable, except that the probability of fuzzy numbers is considered.

Step 2: determine the ideal values.

For positive criteria, the value of $f^{*}$ is equal to the largest fuzzy number values and that of $f^{\circ}$ is equal to the smallest fuzzy number values. And vice versa. The relations are given as follows:

$$
\begin{aligned}
& \tilde{f}_{i}^{z *}=\max _{j} \tilde{f}_{i j}^{z}, \\
& \tilde{f}_{i}^{z^{\circ}}=\min _{j} \tilde{f}_{i j}^{z}, \quad \text { for } i \in I^{b}, \\
& \tilde{f}_{i}^{z^{*}}=\max _{j} \tilde{f}_{i j}^{z}, \\
& \tilde{f}_{i}^{z^{\circ}}=\min _{j} \tilde{f}_{i j}^{z}, \quad \text { for } i \in I^{b} .
\end{aligned}
$$

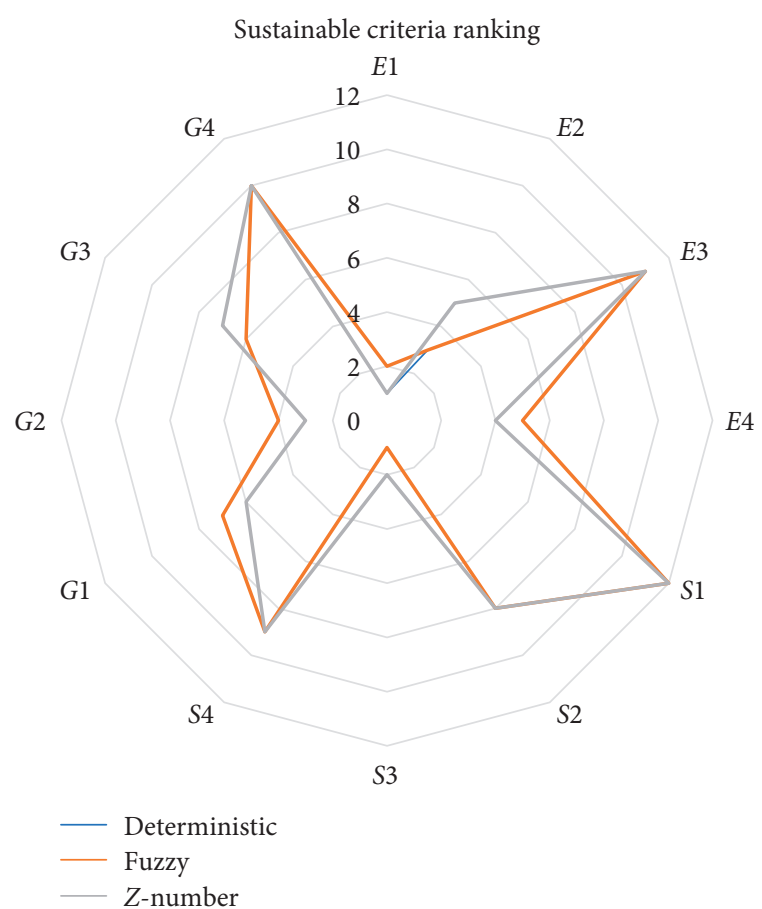

FIgURE 11: Comparison of importance weights of sustainability criteria under different scenarios (in terms of ranking).

Step 3: normalize the decision matrix.

In this step, we will normalize the positive and negative criteria based on the following relationships:

$$
\begin{aligned}
& \tilde{d}_{i j}^{z}=\frac{\left(\tilde{f}_{i}^{z *} \ominus \tilde{f}_{i j}^{z}\right)}{\left(r_{i}^{z *}-l_{i}^{z^{\circ}}\right)}, \quad \text { for } i \in I^{b}, \\
& \tilde{d}_{i j}^{z}=\frac{\left(\tilde{f}_{i j}^{z} \ominus \tilde{f}_{i}^{z *}\right)}{\left(r_{i}^{z^{\circ}}-l_{i}^{z *}\right)}, \quad \text { for } i \in I^{c} .
\end{aligned}
$$

Step 4: determine the values $(\widetilde{S})$ and $(\widetilde{R})$.

In this step, we must first weigh the normal matrix and then obtain the following values:

$$
\begin{aligned}
& \widetilde{S}_{j}^{z}=\sum_{i=1}^{n}\left(\widetilde{w}_{j}^{z} \otimes \tilde{d}_{i j}^{z}\right), \\
& \widetilde{R}_{j}^{z}=\max _{i}\left(\tilde{w}_{j}^{z} \otimes \tilde{d}_{i j}^{z}\right) .
\end{aligned}
$$

Step 5: calculate the VIKOR index $(\widetilde{Q})$.

This index is calculated using the following equation. In this regard, the variable $v$ is the maximum group efficiency. And the number is between 0 and 1 :

$Z$ - number: $\widetilde{Q}_{j}^{z}=v\left(\frac{\widetilde{S}_{j}^{z} \ominus \widetilde{S}^{z *}}{S^{z^{\circ} r}-S^{z * I}}\right) \oplus(1-v)\left(\frac{\widetilde{R}_{j}^{z} \ominus \widetilde{R}^{z *}}{R^{z^{\circ} r}-R^{z * I}}\right)$.

Step 6: defuzzy numbers $\widetilde{R}, \widetilde{S}$, and $\widetilde{Q}$ and sort the options based on $\widetilde{R}, \widetilde{S}$, and $\widetilde{Q}$. 
TABLE 51: The results of the effectiveness and influence of the dimensions and criteria of sustainability in different scenarios.

\begin{tabular}{|c|c|c|c|c|c|c|c|}
\hline Sustainability dimensions & Deterministic & fuzzy & Z-number & Sustainability criteria & Deterministic & Fuzzy & Z-number \\
\hline \multirow{5}{*}{ Economic } & \multirow{5}{*}{ Influence } & \multirow{5}{*}{ Influence } & \multirow{4}{*}{ Influence } & $E 1$ & Influence & Influence & Influence \\
\hline & & & & $E 2$ & Influence & Influence & Influence \\
\hline & & & & E3 & Influence & Influence & Influence \\
\hline & & & & E4 & Influence & Influence & Influence \\
\hline & & & \multirow{4}{*}{ Influence } & $S 1$ & Be-influence & Be-influence & Be-influence \\
\hline \multirow{3}{*}{ Social } & \multirow{3}{*}{ Influence } & \multirow{3}{*}{ Be-influence } & & $S 2$ & Be-influence & Be-influence & Be-influence \\
\hline & & & & $S 3$ & Be-influence & Be-influence & Be-influence \\
\hline & & & & $S 4$ & Be-influence & Be-influence & Be-influence \\
\hline \multirow{4}{*}{ Environment } & \multirow{4}{*}{ Be-influence } & \multirow{4}{*}{ Be-influence } & \multirow{4}{*}{ Be-influence } & G1 & Be-influence & Be-influence & Be-influence \\
\hline & & & & G2 & Influence & Influence & Influence \\
\hline & & & & G3 & Influence & Influence & Influence \\
\hline & & & & G4 & Be-influence & Be-influence & Be-influence \\
\hline
\end{tabular}

First, we defuzzificate the fuzzy numbers by the weighted average:

$$
Z \text { - number defuzzy: } \frac{f+2 g+h}{4} .
$$

Options are ranked based on the defuzzy values. The first rank is given to the lowest values and the last rank is given to the highest values. To choose the best option, we should check the following conditions. The best option is the option that has the lowest $Q$ value, provided that the following conditions are satisfied.

Condition 1. If $A^{(1)}, A^{(2)}$, and $A^{(n)}$ are the first, second, and last options, respectively, based on the value of $Q$, and $n$ indicates the number of options, the following relation must be in place:

$$
\frac{\left[Q\left(A^{(2)}\right)-Q\left(A^{(1)}\right)\right]}{\left[Q\left(A^{(n)}\right)-Q\left(A^{(1)}\right)\right]} \geq \frac{1}{n-1} .
$$

If this condition is not met, a set of options will be selected as the best options as follows.

$$
\text { best alternative }=A 1, A 2, \cdots, A m \text {. }
$$

The maximum value of $m$ is calculated according to the following relation:

$$
Q(A m)-Q(A 1)<\frac{1}{(n-1)} \Longrightarrow Q(A m)<\left(\frac{1}{N-1}\right)+Q(A 1) \text {. }
$$

If Condition 1 is met, condition 2 must also be checked.

Condition 2. Option $A 1$ must be at least in one of the $R$ and $S$ groups of the first rank.

When the second condition is not met, the two $A 1$ and A2 options are recognized as the best options. If both conditions are met, the ranking will be based on $Q$ (the lower the $Q$, the better).
TABLE 52: Project ranking results using the VIKOR approach under different scenarios (first example: 8 projects).

\begin{tabular}{lcccccccccccc}
\hline \multirow{2}{*}{ Project } & \multicolumn{1}{c}{ Deterministic } & \multicolumn{4}{c}{ Fuzzy } & \multicolumn{4}{c}{ Z-number } \\
& $R$ & $S$ & $Q$ & Rank & $R$ & $S$ & $Q$ & Rank & $R$ & $S$ & $Q$ & Rank \\
\hline$P 1$ & 2 & 2 & 1 & 1 & 1 & 2 & 1 & 1 & 1 & 2 & 2 & 1 \\
$P 2$ & 1 & 5 & 2 & 2 & 3 & 5 & 2 & 3 & 2 & 4 & 1 & 2 \\
$P 3$ & 8 & 7 & 7 & $\mathbf{x}$ & 7 & 7 & 8 & 4 & 8 & 7 & 8 & $\mathbf{x}$ \\
$P 4$ & 3 & 4 & 3 & $\mathbf{x}$ & 4 & 4 & 3 & 7 & 3 & 6 & 3 & $\mathbf{x}$ \\
$P 5$ & 6 & 8 & 8 & $\mathbf{x}$ & 8 & 8 & 7 & 8 & 7 & 8 & 6 & $\mathbf{x}$ \\
$P 6$ & 4 & 3 & 5 & $\mathbf{x}$ & 5 & 3 & 4 & 5 & 5 & 3 & 5 & $\mathbf{x}$ \\
$P 7$ & 6 & 6 & 6 & $\mathbf{x}$ & 6 & 6 & 5 & 6 & 6 & 5 & 4 & $\mathbf{x}$ \\
$P 8$ & 5 & 1 & 4 & $\mathbf{x}$ & 2 & 1 & 6 & 2 & 4 & 1 & 7 & $\mathbf{x}$ \\
\hline
\end{tabular}

\section{Case Study}

In order to implement the suggested approach of this paper, a project-based mass production and infrastructural company, based in Iran, has been selected. For filtering the sustainable criteria, two experts and a senior expert have been employed from the project portfolio management department of the company-considering their knowledge of the scopes and objectives of the company-to fill in the information associated with decision-making tables (pairwise comparison matrices and scoring matrix).

Therefore, the identified and categorized sustainable criteria are filtered by two experts and the senior expert of the project portfolio management department of the company. Over this process, each of these experts scores the criteria from 0 to 10 (not-effective to highly effective) according to the objectives and the scopes of the company, after which the integer average of these scores is calculated, and any criterion with a score of over 5 is selected.

In this section, first, the appropriate sustainability criteria for project ranking are identified. Then, using the DEMATEL approach in deterministic, fuzzy, and Znumber conditions, the effectiveness or influence of criteria and the importance weight of sustainability dimensions and criteria have been investigated. Finally, the VIKOR approach in deterministic, fuzzy, and Z-number conditions for project ranking and analysis and comparison of results obtained in deterministic, fuzzy, and Z-number conditions have been used. 
4.1. Filtering Sustainability Criteria. According to Table 4 in the previous section and the opinions of experts, the filtering of sustainability criteria has been done and the following criteria have been selected for project selection (Table 8).

Figure 6 shows the sustainability dimensions and criteria along with related codes.

4.2. The Results of the DEMATEL Numerical Example in Deterministic, Fuzzy, and Z-Number Conditions. After filtering the sustainability criteria in the previous section, in this section, the sustainability dimensions and criteria have been scored using a pairwise comparison matrix by an expert under the deterministic, fuzzy, and Z-number conditions to measure the importance of sustainability dimensions and criteria and calculate the effectiveness of them by DEMATEL approach. In this section, only the calculations related to the Z-DEMATEL approach are performed and the calculations related to the deterministic and fuzzy data are given in Tables 9-14 ).

First, the sustainability dimensions were scored by the expert using the Z-number linguistic variable of Table 6 in the pairwise comparison matrix of Table 15, and then in Table 16, the linguistic variable was matched with the corresponding Z-number value.

In Table 17, the pairwise comparison matrix is normalized, and in Table 18, the total relationship matrix of sustainability dimensions in Z-number conditions is calculated.

Finally, in Table 19, the effectiveness or influence of sustainability dimensions has been calculated, and through it, the importance of sustainability dimensions has been calculated. If $\widetilde{D}_{i}-\widetilde{R}_{i}>0$, the relevant criteria are effective, and if $\widetilde{D}_{i}-\widetilde{R}_{i}<0$, the relevant criteria are influential. Table 19 shows $\widetilde{D}_{i}+\widetilde{R}_{i}$ and. $\widetilde{D}_{i}-\widetilde{R}_{i}$.

Figure 7 shows the importance, effectiveness, influence, and feasibility between the sustainability dimensions. The horizontal axis of the graph shows the importance of the criteria and the vertical axis shows the effectiveness or influence of the dimensions. According to the figure, the economic dimension is the most effective and the environmental dimension is the most influential dimension.

Similarly, to the sustainability dimensions, an expert has examined the sustainability criteria (Tables 20 ).

In Table 9, the pairwise comparison matrix shows the sustainability criteria in the form of linguistic variable and fuzzy numbers, taking into account its corresponding probability. The normal matrix and the total relationship matrix of sustainability criteria are shown in Table 10. Table 21 shows $\widetilde{D}_{i}+\widetilde{R}_{i}$ and $\widetilde{D}_{i}-\widetilde{R}_{i}$ examination of sustainability criteria effectiveness. Also, the importance weights of the sustainability criteria in relation to the sustainability dimensions and the purpose of the problem have been calculated.

Figure 8 shows the importance and effectiveness or influence of the criteria. The horizontal axis of the graph shows the importance of the criteria and the vertical axis shows the effectiveness of the criteria. According to the figure, the existence of basic infrastructure is the most
TABLE 53: Project ranking results using the VIKOR approach under different scenarios (second example: 20 projects).

\begin{tabular}{lcccccccccccc}
\hline \multirow{3}{*}{ Project } & \multicolumn{1}{c}{ Deterministic } & \multicolumn{4}{c}{ Fuzzy } & \multicolumn{4}{c}{ Z-number } \\
& $R$ & $S$ & $Q$ & Rank & $R$ & $S$ & $Q$ & Rank & $R$ & $S$ & $Q$ & Rank \\
\hline$P 1$ & 7 & 9 & 8 & $\mathbf{x}$ & 7 & 8 & 7 & 7 & 13 & 7 & 11 & 11 \\
$P 2$ & 12 & 7 & 11 & $\mathbf{x}$ & 12 & 7 & 12 & 12 & 17 & 8 & 13 & 13 \\
$P 3$ & 9 & 2 & 3 & 3 & 10 & 2 & 5 & 5 & 7 & 3 & 5 & 5 \\
$P 4$ & 4 & 8 & 7 & $\mathbf{x}$ & 4 & 9 & 6 & 6 & 2 & 4 & 3 & 3 \\
$P 5$ & 3 & 10 & 4 & $\mathbf{x}$ & 3 & 10 & 3 & 3 & 6 & 10 & 7 & 7 \\
$P 6$ & 12 & 11 & 14 & $\mathbf{x}$ & 16 & 11 & 14 & 14 & 15 & 16 & 17 & 17 \\
$P 7$ & 19 & 14 & 16 & $\mathbf{x}$ & 18 & 15 & 18 & 18 & 20 & 13 & 19 & 19 \\
$P 8$ & 2 & 1 & 1 & 1 & 2 & 1 & 2 & 2 & 3 & 1 & 1 & 1 \\
$P 9$ & 7 & 4 & 5 & $\mathbf{x}$ & 7 & 4 & 4 & 4 & 5 & 9 & 6 & 6 \\
$P 10$ & 14 & 19 & 18 & $\mathbf{x}$ & 13 & 20 & 16 & 16 & 10 & 19 & 12 & 12 \\
$P 11$ & 4 & 12 & 9 & $\mathbf{x}$ & 4 & 13 & 10 & 10 & 8 & 11 & 9 & 9 \\
$P 12$ & 4 & 13 & 10 & $\mathbf{x}$ & 4 & 12 & 9 & 9 & 9 & 12 & 10 & 10 \\
$P 13$ & 19 & 20 & 20 & $\mathbf{x}$ & 18 & 19 & 19 & 19 & 19 & 17 & 20 & 20 \\
$P 14$ & 18 & 17 & 19 & $\mathbf{x}$ & 20 & 17 & 20 & 20 & 12 & 15 & 14 & 14 \\
$P 15$ & 14 & 16 & 15 & $\mathbf{x}$ & 17 & 16 & 17 & 17 & 16 & 14 & 16 & 16 \\
$P 16$ & 1 & 3 & 2 & 2 & 1 & 3 & 1 & 1 & 1 & 6 & 2 & 2 \\
$P 17$ & 11 & 15 & 13 & $\mathbf{x}$ & 9 & 14 & 11 & 11 & 14 & 18 & 15 & 15 \\
$P 18$ & 9 & 6 & 6 & $\mathbf{x}$ & 10 & 5 & 8 & 8 & 4 & 5 & 4 & 4 \\
$P 19$ & 14 & 18 & 17 & $\mathbf{x}$ & 13 & 18 & 15 & 15 & 18 & 20 & 18 & 18 \\
$P 20$ & 14 & 5 & 12 & $\mathbf{x}$ & 13 & 6 & 13 & 13 & 10 & 2 & 8 & 8 \\
\hline
\end{tabular}

effective index, and the satisfaction of employees and personnel is the most influential index, and also in terms of weight, the profit index will be most important than other criteria.

4.3. Example 1: The Results of the VIKOR Numerical Example in Z-Number Condition, Taking into Account the 8 Proposed Projects. In order to evaluate the projects in this study, the linguistic scoring variable considering its probabilities of Table 7 has been used for qualitative criteria. On the other hand, quantitative criteria such as profit based on the past data are considered as fuzzy numbers with a probability of 1 . Table 22 shows the linguistic scoring variable of sustainability criteria, taking into account the probability of expert opinions for the 8 proposed projects' reliability. The calculations related to deterministic and fuzzy data are given in Tables 23-44.

The linguistic scoring variable matrix of sustainability criteria, according to Table 12, has become the matrix of scoring fuzzy numbers corresponding to them, taking into account the probabilities (quantified scoring matrix). Table 12 shows the normalized values of the evaluation matrix. Table 45 shows the fuzzy and defuzzy values of $S, R$, and $Q$. In this step, the projects are ranked based on the values of $R, S$, and $Q$.

According to the table, only the first and second projects can be ranked, and other projects cannot be ranked and compared due to their scores.

4.4. Example 2: The Results of the VIKOR Numerical Example in Deterministic, Fuzzy, and Z-Number Conditions, Taking into Account 20 Proposed Projects. In this example, we assume that all sustainability criteria are qualitative criteria. 


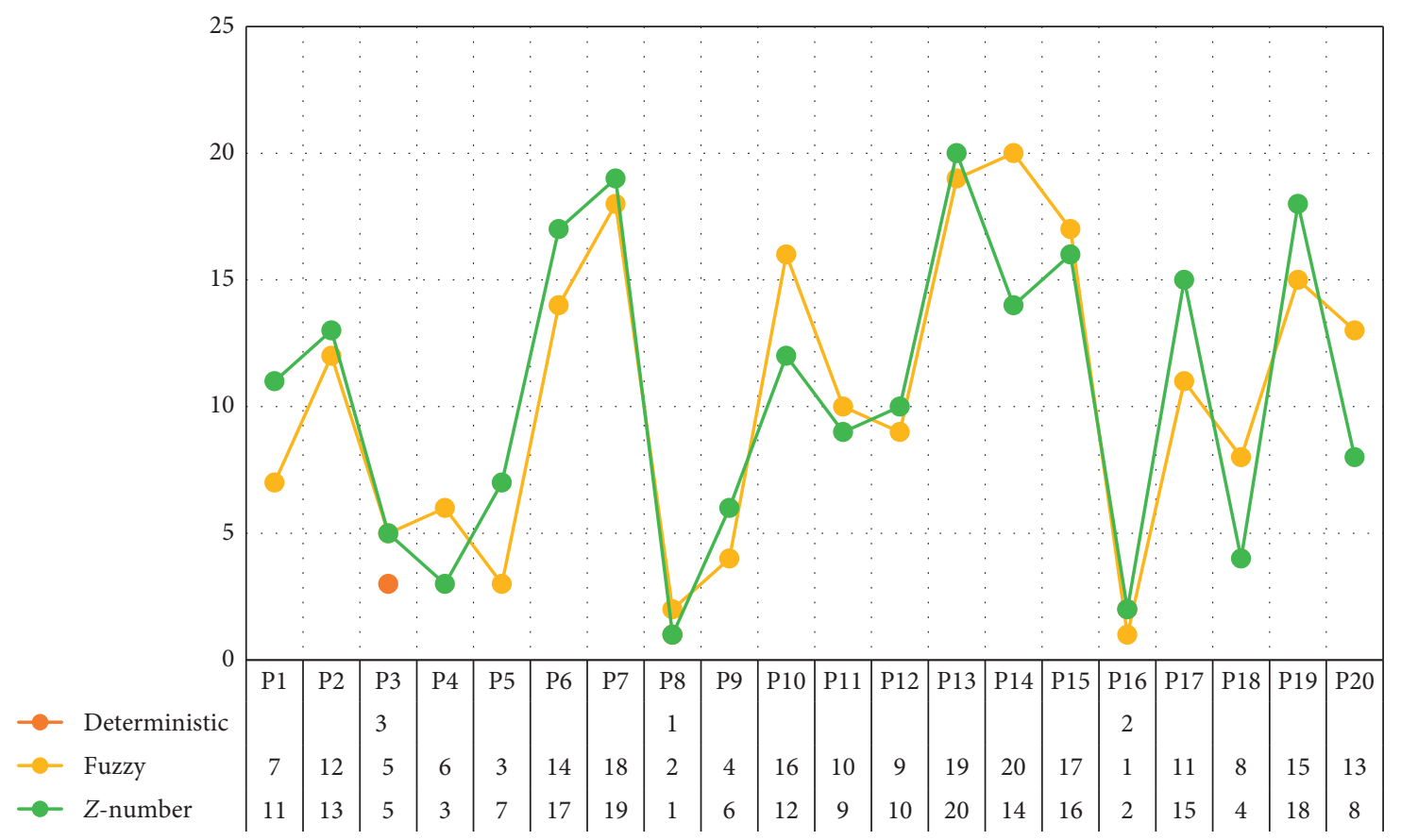

FIGURE 12: Project ranking results using the VIKOR approach under different scenarios (second example: 20 projects).

Therefore, according to the first example, to evaluate the projects, the probabilistic linguistic scoring variable of Table 7 has been used. Table 46 shows the sustainability criteria considering the probability of relying on expert opinions for the 20 proposed projects.

Similarly, to the first example, the quantified linguistic variable matrixes corresponding to Table 46 as well as the normalized matrix are shown in Tables 47 and 48 , respectively. Table 49 shows the fuzzy and defuzzy values of $S$, $R$, and $Q$. In this step, the projects are ranked based on the values of $R, S$, and $Q$.

According to the table, unlike the first example, according to the scoring data, all projects are ranked.

\section{Discussion and Analysis of Results}

In this section, the results of the two numerical examples presented are analyzed. First, a comparison of ranks, effectiveness, and influence and value of the obtained weights of sustainability dimensions and criteria under deterministic, fuzzy, and Z-number conditions are discussed, considering two numerical examples of 8 projects and 20 projects.

\subsection{Comparison of the Weights Obtained from Sustainability} Dimensions and Criteria under Different Conditions. Table 50 summarizes the results of the weights obtained and ranks the sustainability dimensions and criteria under different deterministic, fuzzy, and Z-number scenarios.

According to the table above and Figure 9 (comparison under different scenarios), the numerical value of the weights has changed, but in terms of ranking the importance of weights, the economic dimension in each scenario has the highest weight and the social dimension has the lowest weight.

Also, in Figures 10 and 11, it is observed that the weights of the sustainability criteria differ in terms of both weight and ranking under different scenarios.

It can be concluded that different responses are obtained under different scenarios, and on the other hand, as it moves from a deterministic scenario to fuzzy and Z-number scenarios, due to the uncertainty in the judgments of experts and the nature of fuzzy numbers and Z-number, it can present more reliable results.

Also, in Table 51, the degree of influence and effectiveness of dimensions and criteria of sustainability in different scenarios is stated and it is observed that different scenarios have almost no effect on the effectiveness and influence of sustainability criteria, and only in the social dimension in fuzzy mode, its impact changes.

The following is an analysis of the ranking results of projects resulting from the VIKOR approach under different scenarios (the first example, which includes 8 projects, and the second example, which includes 20 projects).

Table 52 shows the final ranking of projects (which includes 8 projects) based on $R, S$, and $Q$ values under different scenarios. It is observed that, under different scenarios, the values of $R, S$, and $Q$ are different and the ranking of projects is the same in deterministic and Znumber scenarios but different in fuzzy scenario. In order to further analyze the ranking results, the second example has been examined according to the 20 proposed projects. Therefore, in Table 53, the ranking of projects and $R, S$, and $Q$ values for 20 projects under different scenarios are given. According to the table, both $R, S$, and $Q$ values and the final ranking of projects in different scenarios are different. 
Figure 12 shows the ranking of projects in the second example under different scenarios. It can be seen that the graphs do not match and the results of project rankings vary under different scenarios. Therefore, it can be concluded that the level of confidence in the data and judgments of experts can affect our decisions and ranking, and the more it moves from scenario with deterministic data to scenario with fuzzy and Z-number data, the closer it can get to the real world. Because Z-number also considers the reliability of fuzzy data, the results of this approach will be more reliable.

5.1.1. Implications for theory. According to the obtained results, the following items can be stated as theoretical applications of the method:

(1) Using the DEMATEL approach under fuzzy data considering their reliability and probability (Znumber), the interactive effects of sustainable criteria (influence and be-influence) and their significance weights can be determined considering uncertainties caused by human judgments. This results in more reliable and more realistic results compared to certain and fuzzy data due to the consideration of the reliability of data. Moreover, given that different results have been attained for different scenarios (certain, fuzzy, and Z-number), the superiority of Znumber data is proved clearly. Accordingly, ZDEMATEL is an effective approach for the investigation of (influence and be-influence) and the calculation of significance weights under uncertainties.

(2) Given the obtained significance weights from ZDEMATEL $(0.378,0.255$, and 0.367 for economic, social, and environmental aspects, respectively) and the similarity of sustainability weights, which is indicative of the importance of sustainable criteria in today's life, companies thrive to take these other factors into consideration apart from economic aspects to be able to survive in the competitive market.

(3) By means of the VIKOR approach under fuzzy data considering their reliability and probability (Znumber), the candidate projects can be ranked based on sustainable criteria while also considering uncertainties in the opinions of experts. Consequently, more reliable results are obtained since uncertainties are involved in the reliability of data, making the results more realistic. Owing to the fact that different results are obtained under different scenarios (certain, fuzzy, and Z-number), which undoubtedly affects the ranking of the projects, the importance of $Z$ number data is more clearly shown by the realistic results it yields. Therefore, Z-VIKOR is shown to be an efficient approach for ranking projects in uncertain conditions.

(4) Given the uncertainties in judgments of experts, making use of data that indicate these uncertainties can help improve decision-making. Due to the difference in the results obtained for different scenarios (certain, fuzzy, and Z-number) for the calculation of significance weights of the sustainable criteria, it can be concluded that Z-number information yields far more reliable and more realistic results as it is capable of considering reliability and probability of fuzzy data.

5.1.2. Implications for practice. Currently, institutionalization of project management in project-based organizations and employing its benefits in the long term is one of the major concerns in the field of project management, leading to the fact that specific project management techniques are often ignored. In this respect, project portfolio management is an efficient tool to help enhance the efficiency and efficacy of the projects of organizations. The main tools of implementing the strategies of project-based organizations, including selecting and effectively carrying out the projects, play a key role in the success of organizations. Therefore, this research provides an effective tool for ranking and selecting appropriate projects according to sustainable criteria and strategic organizational objectives.

\section{Conclusion and Future Recommendation}

6.1. Managerial Analysis of Sustainable Development of Research. In this study, first, the sustainability criteria effective in selecting the project portfolio and validating these criteria were identified. In order to achieve this goal, a methodology was used to identify, classify, and prioritize sustainability criteria. Based on this methodology, 49 project sustainability criteria were identified through the literature review. These criteria were classified using a sustainable breakdown structure. Based on this structure, the criteria were categorized into three economic, social, and environmental dimensions. In the last step, the opinions of the experts were used to validate and highlight each of them so that these criteria could be filtered. After filtering, the DEMATEL method was used to determine the weight and importance of each sustainability dimension and criteria, as well as the degree of effectiveness or influence of them in different scenarios of deterministic, fuzzy, and Z-number. The results show that the weight of the filtered criteria in $\mathrm{Z}$ number is between 0.045 and 0.14 and very close to each other; this indicates the importance of all these filtered criteria as well as the importance of all three dimensions of sustainability.

\subsection{Managerial Analysis of Research Uncertainty.} Although quantitative traditional methods provide precise solutions, they are not useful for solving real-world problems due to the complexity of human factors and their judgments. In real-world programs, decision-making issues must be done with uncertainty because goals, constraints, and criteria are ambiguous. Therefore, fuzzy management science, while creating flexibility in the model, enters data such as knowledge, experience, and human judgment in the model and provides fully practical answers. In this study, according to the importance of the mentioned topics, first, the sustainability 
criteria were filtered through a questionnaire, and then according to the pairwise comparison matrix of sustainability dimensions and criteria, their weights were calculated by experts. Project rankings were calculated through the DEMATEL-VIKOR approach in deterministic, fuzzy, and Znumber conditions using the decision-making matrix of sustainability criteria for projects. Different results and different rankings were found. Based on the results obtained, it can be seen that the more it moves from the deterministic to fuzzy conditions, and fuzzy conditions considering probabilities (Z-number), the more accurate the results are according to the uncertainties in the judgments and opinions of experts. Therefore, the Z-DEMATEL-VIKOR approach can be a suitable and reliable approach for calculating the ranking of sustainable projects.

\subsection{Practical Applications and Future Recommendation.} Regarding the limitations of the present paper, the following items can be mentioned: (1) limitation in the number of experts for the provision of the questionnaires due to restrictions of the company under consideration (only one senior expert has been used), (2) limited time of the expert to answer the questionnaires given a large number of provided questionnaires, which has potentially affected the accuracy of the responses of the mentioned expert, and (3) limitations in the accessibility of data and information of the candidate projects.

The proposed Z-DEMATEL-VIKOR approach and the advantages it provides for project-based companies to evaluate and rank their projects according to sustainable criteria is the key benefit of the present research work. Accordingly, the proposed approach can be an efficient decision-making tool to select the most appropriate projects aligned with the objectives and scopes of the company.

In this study, an attempt was made to identify the sustainability criteria and use them in the proposed model to partially consider the principles of sustainability in the project portfolio selection process. But it will take a lot of effort to make full use of sustainable development in this process. For future research, the following suggestions are provided:

(1) Using these sustainability criteria in different frameworks, models, and approaches: select the projects in the frameworks and models that have been proposed to select the project portfolio in which these criteria have been used; therefore, it is suggested to use these identified criteria in different approaches and frameworks for project selection.

(2) Using the principles of sustainable development in all project portfolio management processes: as mentioned earlier, there are three process groups in portfolio management, which are definition production group, alignment process group, and approval and control process group. The project portfolio selection is in the alignment group. In order to achieve sustainable portfolio management, it is suggested also to consider the principles of sustainable development in the other two process groups.

(3) Creating a framework for evaluating the sustainability of projects during the project life cycle: considering the principles of sustainability in the project selection phase will help select sustainable projects. But in other phases of the project (needs assessment, design, construction, initial testing, delivery, and operation), it also needs frameworks that assess the sustainability of projects at different stages.

(4) Development of effective sustainability criteria in project portfolio selection: in this study, a number of effective sustainability criteria in project portfolio selection were identified, classified, accredited, and prioritized. In future research, these criteria can be developed and other methods such as analytic hierarchy process (AHP) and analytic network process (ANP) can be used to prioritize them.

(5) Using other uncertainty approaches and comparing it with the uncertainty approach of this research: in this study, using fuzzy logic and Z-number approach, the results obtained from them were compared. It is suggested that using other approaches of uncertainty such as grey theory and grey theory with the probability of its reliability, the results are analyzed and compared with the results of this study.

\section{Data Availability}

The data used to support the findings of this study are available within the article.

\section{Conflicts of Interest}

The authors declare that they have no conflicts of interest.

\section{References}

[1] K. Khalili-Damghani and M. Tavana, "A comprehensive framework for sustainable project portfolio selection based on structural equation modeling," Project Management Journal, vol. 45, no. 2, pp. 83-97, 2014.

[2] K. Khalili-Damghani and S. Sadi-Nezhad, "A hybrid fuzzy multiple criteria group decision making approach for sustainable project selection," Applied Soft Computing, vol. 13, no. 1, pp. 339-352, 2013

[3] K. Khalili-Damghani, S. Sadi-Nezhad, F. H. Lotfi, and M. Tavana, "A hybrid fuzzy rule-based multi-criteria framework for sustainable project portfolio selection," Information Sciences, vol. 220, pp. 442-462, 2013.

[4] W. Ho, X. Xu, and P. K. Dey, "Multi-criteria decision making approaches for supplier evaluation and selection: a literature review," European Journal of Operational Research, vol. 202, no. 1, pp. 16-24, 2010.

[5] B. Kang, P. Zhang, Z. Gao, G. Chhipi-Shrestha, K. Hewage, and R. Sadiq, "Environmental assessment under uncertainty using Dempster-Shafer theory and Z-numbers," Journal of Ambient Intelligence and Humanized Computing, vol. 11, no. 5, pp. 2041-2060, 2019.

[6] Z. Chen and W. Yang, "A new multiple criteria decision making method based on intuitionistic fuzzy information," 
Expert Systems with Applications, vol. 39, no. 4, pp. 43284334, 2012.

[7] D. Demirli, K.-W. Shen, J.-Q. Wang, and T.-L. Wang, "Multicriteria PROMETHEE method based on possibility degree with Z-numbers under uncertain linguistic environment," Journal of Ambient Intelligence and Humanized Computing, vol. 11, pp. 1-15, 2019.

[8] S. Hendiani and M. Bagherpour, "Development of sustainability index using Z-numbers: a new possibilistic hierarchical model in the context of Z-information," Environment, Development and Sustainability, vol. 22, no. 7, pp. 6077-6109, 2020.

[9] G. Büyüközkan and G. Çifçi, "A novel hybrid MCDM approach based on fuzzy DEMATEL, fuzzy ANP and fuzzy TOPSIS to evaluate green suppliers," Expert Systems with Applications, vol. 39, no. 3, pp. 3000-3011, 2012.

[10] S. Ebrahimnejad, S. M. Mousavi, R. Tavakkoli-Moghaddam, H. Hashemi, and B. Vahdani, "A novel two-phase group decision making approach for construction project selection in a fuzzy environment," Applied Mathematical Modelling, vol. 36, no. 9, pp. 4197-4217, 2012.

[11] R. G. Cooper, S. J. Edgett, and E. J. Kleinschmidt, Standard of Portfolio Management, Portfolio Management, Pegasus, NY, USA, 2009.

[12] A. RezaHoseini, S. F. Ghannadpour, and M. Hemmati, "A comprehensive mathematical model for resource-constrained multi-objective project portfolio selection and scheduling considering sustainability and projects splitting," Journal of Cleaner Production, vol. 269, p. 122073, 2020a.

[13] A. Mahmoudi, M. Abbasi, X. Deng, M. Ikram, and S. Yeganeh, "A novel model for risk management of outsourced construction projects using decision-making methods: a case study," Grey Systems: Theory and Application, vol. 10, no. 2, 2020.

[14] F. H. Rad and S. M. Rowzan, "Designing a hybrid system dynamic model for analyzing the impact of strategic alignment on project portfolio selection," Simulation Modelling Practice and Theory, vol. 89, pp. 175-194, 2018.

[15] A. Smith-Perera, M. García-Melón, R. Poveda-Bautista, and J.-P. Pastor-Ferrando, “A Project Strategic Index proposal for portfolio selection in electrical company based on the Analytic Network Process," Renewable and Sustainable Energy Reviews, vol. 14, no. 6, pp. 1569-1579, 2010.

[16] Y. Wu, C. Xu, Y. Ke, Y. Tao, and X. Li, "Portfolio optimization of renewable energy projects under type-2 fuzzy environment with sustainability perspective," Computers \& Industrial Engineering, vol. 133, pp. 69-82, 2019.

[17] Y. Wu, C. Xu, Y. Ke, K. Chen, and X. Sun, "An intuitionistic fuzzy multi-criteria framework for large-scale rooftop PV project portfolio selection: case study in Zhejiang, China," Energy, vol. 143, pp. 295-309, 2018.

[18] S. Jones, M. Tefe, and S. Appiah-Opoku, "Proposed framework for sustainability screening of urban transport projects in developing countries: a case study of Accra, Ghana," Transportation Research Part A: Policy and Practice, vol. 49, pp. 21-34, 2013.

[19] S. Kudratova, X. Huang, and X. Zhou, "Sustainable project selection: optimal project selection considering sustainability under reinvestment strategy," Journal of Cleaner Production, vol. 203, pp. 469-481, 2018.

[20] J. Ma, J. D. Harstvedt, R. Jaradat, and B. Smith, "Sustainability driven multi-criteria project portfolio selection under uncertain decision-making environment," Computers \& Industrial Engineering, vol. 140, Article ID 106236, 2020.
[21] N. Dobrovolskienė and R. Tamošiūniené, "Sustainabilityoriented financial resource allocation in a project portfolio through multi-criteria decision-making," Sustainability, vol. 8 , no. 5 , p. $485,2016$.

[22] A. J. G. Silvius and R. Schipper, "A conceptual model for exploring the relationship between sustainability and project success," Procedia Computer Science, vol. 64, pp. 334-342, 2015.

[23] C.-Y. Duan, H.-C. Liu, L.-J. Zhang, and H. Shi, “An extended alternative queuing method with linguistic $Z$-numbers and its application for green supplier selection and order allocation," International Journal of Fuzzy Systems, vol. 21, no. 8, pp. 2510-2523, 2019.

[24] A.-Y. Chang and Y.-T. Cheng, "Analysis model of the sustainability development of manufacturing small and mediumsized enterprises in Taiwan," Journal of Cleaner Production, vol. 207, pp. 458-473, 2019.

[25] M. Ikram, P. Zhou, S. A. A. Shah, and G. Q. Liu, "Do environmental management systems help improve corporate sustainable development? Evidence from manufacturing companies in Pakistan," Journal of Cleaner Production, vol. 226, pp. 628-641, 2019.

[26] M. Ikram, R. Sroufe, and Q. Zhang, "Prioritizing and overcoming barriers to integrated management system (IMS) implementation using AHP and G-TOPSIS," Journal of Cleaner Production, vol. 254, Article ID 120121, 2020a.

[27] M. Ikram, Q. Zhang, R. Sroufe, and M. Ferasso, "The social dimensions of corporate sustainability: an integrative framework including COVID-19 insights," Sustainability, vol. 12, no. 20, p. 8747, 2020 b.

[28] R. G. Cooper, Winning at New Products, Addison-Wesley, Reading, MA, USA, 1993.

[29] N. P. Archer and F. Ghasemzadeh, Project Portfolio Selection Techniques: A Review and a Suggested Integrated Approach, Michael G. DeGroote School of Business, McMaster University, Hamilton, Ontario, Canada, 1996.

[30] J. W. Lee and S. H. Kim, "Using analytic network process and goal programming for interdependent information system project selection," Computers \& Operations Research, vol. 27, no. 4, pp. 367-382, 2000.

[31] L. M. Meade and A. Presley, "R\&D project selection using the analytic network process," IEEE Transactions on Engineering Management, vol. 49, no. 1, pp. 59-66, 2002.

[32] M. Enea and T. Piazza, "Project selection by constrained fuzzy AHP,” Fuzzy Optimization and Decision Making, vol. 3, no. 1, pp. 39-62, 2004.

[33] D.-Y. Chang, "Applications of the extent analysis method on fuzzy AHP," European Journal of Operational Research, vol. 95, no. 3, pp. 649-655, 1996.

[34] D.-Y. Chang and L. L. Zhang, "Extent analysis and synthetic decision," Optimization Techniques and Application, vol. 1, pp. 352-355, 1992.

[35] R. P. Mohanty, R. Agarwal, A. K. Choudhury, and M. K. Tiwari, "A fuzzy ANP-based approach to R\&D project selection: a case study," International Journal of Production Research, vol. 43, no. 24, pp. 5199-5216, 2005.

[36] E. W. L. Cheng and H. Li, "Analytic network process applied to project selection," Journal of Construction Engineering and Management, vol. 131, no. 4, pp. 459-466, 2005.

[37] C.-C. Huang, P.-Y. Chu, and Y.-H. Chiang, "A fuzzy AHP application in government-sponsored R\&D project selection," Omega, vol. 36, no. 6, pp. 1038-1052, 2008.

[38] P. Aragonés-Beltrán, F. Chaparro-González, J. P. PastorFerrando, and F. Rodríguez-Pozo, "An ANP-based approach 
for the selection of photovoltaic solar power plant investment projects," Renewable and Sustainable Energy Reviews, vol. 14, no. 1, pp. 249-264, 2010.

[39] M. P. Amiri, "Project selection for oil-fields development by using the AHP and fuzzy TOPSIS methods," Expert Systems with Applications, vol. 37, no. 9, pp. 6218-6224, 2010.

[40] J. R. San Cristóbal, "Multi-criteria decision-making in the selection of a renewable energy project in Spain: the Vikor method," Renewable Energy, vol. 36, no. 2, pp. 498-502, 2011.

[41] M. M. Fouladgar, A. Yazdani-Chamzini, S. H. Yakhchali, M. H. Ghasempourabadi, and N. Badri, "Project portfolio selection using VIKOR technique under Fuzzy environment," in Proceedings of the 2nd International Conference on Construction and Project Management, pp. 236-240, Singapore, 2011.

[42] B. Daneshvar Rouyendegh and S. Erol, "Selecting the best project using the fuzzy ELECTRE method," Mathematical Problems in Engineering, vol. 2012, Article ID 790142, 12 pages, 2012.

[43] P. Aragonés-Beltrán, F. Chaparro-González, J.-P. PastorFerrando, and A. Pla-Rubio, "An AHP (Analytic Hierarchy Process)/ANP -based multi-criteria decision approach for the selection of solar-thermal power plant investment projects," Energy, vol. 66, pp. 222-238, 2014.

[44] O. Taylan, A. O. Bafail, R. M. S. Abdulaal, and M. R. Kabli, "Construction projects selection and risk assessment by fuzzy AHP and fuzzy TOPSIS methodologies," Applied Soft Computing, vol. 17, pp. 105-116, 2014.

[45] M. A. Ortíz, H. A. Felizzola, and S. N. Isaza, “A contrast between DEMATEL-ANP and ANP methods for six sigma project selection: a case study in healthcare industry," BMC Medical Informatics and Decision Making, vol. 15, no. S3, p. S3, 2015.

[46] G. Polat, A. Damci, A. P. Gurgun, and I. Demirli, "Urban renewal project selection using the integration of AHP and PROMETHEE approaches," Procedia Engineering, vol. 164, pp. 339-346, 2016.

[47] M. Hamurcu and T. Eren, "A fuzzy analytical network process approach to the selection of the rail system projects," Sigma Journal of Engineering and Natural Science, vol. 9, no. 4, pp. 415-426, 2018.

[48] H. Jafarzadeh, P. Akbari, and B. Abedin, "A methodology for project portfolio selection under criteria prioritisation, uncertainty and projects interdependency - combination of fuzzy QFD and DEA," Expert Systems with Applications, vol. 110, pp. 237-249, 2018.

[49] G. Polat, H. Turkoglu, H. Turkoglu, and A. Damci, "A comparative study on selecting urban renewal project via different MADM methods," Journal of Construction Engineering, Management \& Innovation, vol. 2, no. 3, pp. 131-143, 2019.

[50] A. Shaygan and Ö. M. Testik, "A fuzzy AHP-based methodology for project prioritization and selection," Soft Computing, vol. 23, no. 4, pp. 1309-1319, 2019.

[51] M. Tavana, G. Khosrojerdi, H. Mina, and A. Rahman, "A hybrid mathematical programming model for optimal project portfolio selection using fuzzy inference system and analytic hierarchy process," Evaluation and Program Planning, vol. 77, Article ID 101703, 2019.

[52] A. K. Brahma and D. K. Mitra, "Fuzzy AHP and Fuzzy VIKOR Approach modelling for flood control project selection," International Journal of Applied Engineering Research, vol. 14, no. 17, pp. 3579-35889, 2019.
[53] B. Han, X.-X. Zhang, and Y. Yi, "Multi-criteria project selection using fuzzy preference relations based AHP and TOPSIS," in Proceedings of the 2019a Chinese Control and Decision Conference (CCDC), pp. 3809-3816, IEEE, 2019.

[54] N. Kashyap and R. Garg, "Evaluation and selection of R\&D projects using multi-criteria decision making method," in Proceedings of the 2019 9th International Conference on Cloud Computing, pp. 371-376, IEEE, Noida, India, 2019.

[55] H. A. T. Leão, E. D. Canedo, P. H. T. Costa, M. V. Okimoto, and G. A. Santos, "Use of AHP and promethee for research project portfolio selection," in Proceedings of the International Conference on Computational Science and its Applications, pp. 504-517, Springer, Saint Petersburg, Russia, 2019.

[56] A. S. Yalcin, H. S. Kilic, and E. Guler, "Research and development project selection via IF-DEMATEL and IF-TOPSIS," Intelligent and Fuzzy Systems, pp. 625-633, Springer, New York, NY, USA, 2019.

[57] A. K. Yazdi, A. R. Komijan, P. F. Wanke, and S. Sardar, "Oil project selection in Iran: a hybrid MADM approach in an uncertain environment," Applied Soft Computing, vol. 88, Article ID 106066, 2020.

[58] H. C. Liu, J. X. You, M. M. Shan, and Q. Su, "Systematic failure mode and effect analysis using a hybrid multiple criteria decision-making approach," Total Quality Management \& Business Excellence, vol. 30, no. 5-6, pp. 537-564, 2019.

[59] C. Xu, Y. Wu, and S. Dai, "What are the critical barriers to the development of hydrogen refueling stations in China? A modified fuzzy DEMATEL approach," Energy Policy, vol. 142, Article ID 111495, 2020.

[60] S. Jiang, H. Shi, W. Lin, and H.-C. Liu, "A large group linguistic Z-DEMATEL approach for identifying key performance indicators in hospital performance management," Applied Soft Computing, vol. 86, Article ID 105900, 2020.

[61] M. Akram, C. Kahraman, and K. Zahid, "Group decisionmaking based on complex spherical fuzzy VIKOR approach," Knowledge-Based Systems, vol. 216, Article ID 106793, 2021.

[62] L. A. Zadeh, "On fuzzy algorithms," Fuzzy Sets, Fuzzy Logic, and Fuzzy Systems, vol. 36, no. 2, pp. 127-147, 1995.

[63] F. Xiao, "A hybrid fuzzy soft sets decision making method in medical diagnosis,” IEEE Access, vol. 6, pp. 25300-25312, 2018.

[64] L. Fei, H. Wang, L. Chen, and Y. J. I. Deng, "A new vector valued similarity measure for intuitionistic fuzzy sets based on," OWA Operators, vol. 16, no. 3, pp. 113-126, 2019.

[65] Y. Han, Y. Deng, Z. Cao, and C.-T. Lin, “An interval-valued pythagorean prioritized operator-based game theoretical framework with its applications in multicriteria group decision making," Neural Computing and Applications, vol. 32, no. 12, pp. 7641-7659, 2019.

[66] Y.-M. Wang, J.-B. Yang, D.-L. Xu, K.-S. Chin, and systems, "On the centroids of fuzzy numbers," Fuzzy Sets and Systems, vol. 157, no. 7, pp. 919-926, 2006.

[67] L. A. Zadeh, "A note on Z-numbers," Information Sciences, vol. 181, no. 14, pp. 2923-2932, 2011.

[68] K. Mahler, "An unsolved problem on the powers of $3 / 2$," Journal of the Australian Mathematical Society, vol. 8, no. 2, pp. 313-321, 1968.

[69] H. Aboutorab, M. Saberi, M. R. Asadabadi, O. Hussain, and E. J. E. Chang, "ZBWM: The Z-number extension of Best Worst Method and its application for supplier development," Energy, vol. 107, pp. 115-125, 2018.

[70] N. Marhamati, E. K. Buxton, and S. Rahimi, "Integration of Znumbers and Bayesian decision theory: a hybrid approach to 
decision making under uncertainty and imprecision," Applied Soft Computing, vol. 72, pp. 273-290, 2018.

[71] K.-W. Shen and J.-Q. Wang, "Z-VIKOR method based on a new comprehensive weighted distance measure of Znumber and its application," IEEE Transactions on Fuzzy Systems, vol. 26, no. 6, pp. 3232-3245, 2018.

[72] R. Yazdanparast, R. Tavakkoli-Moghaddam, R. Heidari, and L. Aliabadi, "A hybrid z-Number data envelopment analysis and neural network for assessment of supply chain resilience: a case study," Central European Journal of Operations Research, pp. 1-21, 2018.

[73] A. Jirofti and A. A. Najafi, "Portfolio selection using Znumber theory: two solution methodologies," International Journal of Fuzzy Systems, vol. 20, no. 8, pp. 2484-2496, 2018.

[74] A. R. Hoseini, S. F. Ghannadpour, and R. Ghamari, "Sustainable supplier selection by a new possibilistic hierarchical model in the context of Z-information," Journal of Ambient Intelligence and Humanized Computing, vol. 11, no. 11, pp. 4827-4853, 2020.

[75] A. Shemshadi, H. Shirazi, M. Toreihi, and M. J. Tarokh, "A fuzzy VIKOR method for supplier selection based on entropy measure for objective weighting," Expert Systems with Applications, vol. 38, no. 10, pp. 12160-12167, 2011.

[76] S. Hendiani and M. Bagherpour, "Development of sustainability index using Z-numbers: a new possibilistic hierarchical model in the context of Z-information," vol. 22, no. 7, pp. 6077-6109, 2019.

[77] A. Hargreaves and D. Fink, Sustainable leadership, Vol. 6, John Wiley \& Sons, Hoboken, NJ, USA, 2012.

[78] A. G. Silvius, R. P. Schipper, and S. Studies, "Sustainability in project management competencies: analyzing the competence gap of project managers," Journal of Human Resource and Sustainability Studies, vol. 2, no. 2, pp. 40-58, 2014.

[79] G. Fernández-Sánchez and F. Rodríguez-López, “A methodology to identify sustainability indicators in construction project management-Application to infrastructure projects in Spain," Ecological Indicators, vol. 10, no. 6, pp. 1193-1201, 2010.

[80] C. J. Lin and W. W. Wu, "A causal analytical method for group decision-making under fuzzy environment," Expert Systems with Applications, vol. 34, no. 1, pp. 205-213, 2008. 